TRANSACTIONS OF THE

AMERICAN MATHEMATICAL SOCIETY

Volume 363, Number 10, October 2011, Pages 5131-5178

S 0002-9947(2011)05282-7

Article electronically published on May 4, 2011

\title{
LARGE SOLUTIONS FOR YAMABE AND SIMILAR PROBLEMS ON DOMAINS IN RIEMANNIAN MANIFOLDS
}

\author{
MARTIN DINDOŠ
}

\begin{abstract}
We present a unified approach to study large positive solutions (i.e., $u(x) \rightarrow \infty$ as $x \rightarrow \partial \Omega$ ) of the equation $\Delta u+h u-k \psi(u)=-f$ in an arbitrary domain $\Omega$. We assume $\psi(u)$ is convex and grows sufficiently fast as $u \rightarrow \infty$. Equations of this type arise in geometry (Yamabe problem, two dimensional curvature equation) and probability (superdiffusion). We prove that both existence and uniqueness are local properties of points of the boundary $\partial \Omega$; i.e., they depend only on properties of $\Omega$ in arbitrarily small neighborhoods of each boundary point. We also find several new necessary and sufficient conditions for existence and uniqueness of large solutions including an existence theorem on domains with fractal boundaries.
\end{abstract}

\section{INTRODUCTION}

In this paper we study positive solutions of the problem

$$
\begin{aligned}
\Delta u+h u-k \psi(u) & =-f \text { in } \Omega, \\
u(x) \rightarrow \infty \quad \text { as } \delta(x) & =\operatorname{dist}(x, \partial \Omega) \rightarrow 0
\end{aligned}
$$

on domains $\Omega$ with nonempty (possibly) arbitrary boundary in a compact Riemannian manifold $M$ without boundary of dimension $n=\operatorname{dim} M \geq 2$. Here $\Delta$ is the Laplace-Beltrami operator given by the Riemannian metric on $M$. We assume that the metric tensor $g_{i j}$ on $M$ is at least of class $C^{2}(M)$; hence $M$ has well-defined scalar curvature of class $C(M)$. The function $\psi$ in (1.1) is assumed to be well defined on all nonnegative numbers, vanishing at zero, increasing, convex and growing sufficiently fast as $u \rightarrow \infty$. The precise conditions will be given later. The functions $h, k, f$ are assumed to be sufficiently smooth, and $k, f \geq 0$ with $k$ bounded away from zero near the boundary $\partial \Omega$.

It turns out that equation (11) for various functions $\psi$ plays an important role in geometry, probability, etc. Two classical examples arising in geometry are the Yamabe equation and the two dimensional curvature equation mentioned below. One motivation we had writing this paper was to show that these problems with seemingly very different functions $\psi$ can be given very similar treatment.

Equation (1) with $\psi(u)=u^{(n+2) /(n-2)}$ arises in the problem of conformal change of metric in dimensions 3 and more and is known as the Yamabe problem. Let $g$, $g^{\prime}$ be two conformally related Riemannian metrics. The conformal relationship will

Received by the editors August 1, 2008 and, in revised form, May 30, 2009 and May 31, 2009. 2000 Mathematics Subject Classification. Primary 35J25, 35J60; Secondary 53C21.

Key words and phrases. Elliptic equations, large solutions.

The author was supported in part by EPSRC grant EP/F014589/1-253000 RA0347.

(C)2011 American Mathematical Society Reverts to public domain 28 years from publication 
be written as $g^{\prime}=u^{4 /(n-2)} g$. Denote by $R^{\prime}, R$ their scalar curvature functions. These are related by the equation

$$
\Delta u-\frac{n-2}{4(n-1)} R u+\frac{n-2}{4(n-1)} R^{\prime} u^{(n+2) /(n-2)}=0 .
$$

Here $\Delta$ is the Laplace-Beltrami operator in the metric $g$. The first two terms on the right hand side of (2) are known as the "conformal Laplacian", and the corresponding operator will be denoted by $\mathcal{L}$. Clearly equation (2) is a special case of (11), provided $R^{\prime} \leq 0$ and $R^{\prime}<0$ near the boundary $\partial \Omega$. In this light equation (2) with boundary data as in (1) can be seen as a problem of finding a complete metric $g^{\prime}$ in $\Omega$ with given nonnegative scalar curvature $R^{\prime}$ such that $g^{\prime}$ is conformally related to the background metric $g$ in $M$. The most typical example we want to consider occurs when $R^{\prime}$ is constant and negative. The meaning of the words complete metric is that all geodesics of $g^{\prime}$ in $\Omega$ never intersect the boundary $\partial \Omega$ of $\Omega$; i.e., $\left(\Omega, g^{\prime}\right)$ is geodesically complete. This problem has been considered in many papers both on compact and noncompact manifolds, in particular in [2], 3], 4, 28], 46], 33], 34] [26], most recently in [24], 25] and also elsewhere. The problem for $R^{\prime} \geq 0$, which is of a different nature and not considered here, is addressed in [23], 6], 45], 46], 27], 30, 31, 29], 13] and elsewhere.

If the same problem is considered in two dimensions, a different equation arises. In this case we look for $g^{\prime}=e^{2 u} g$ where $u$ solves

$$
\Delta u+K^{\prime} e^{2 u}=K
$$

Here $K$ is curvature in the original metric and $K^{\prime}$ in the new metric. This problem for $K^{\prime}=-1$ was extensively considered in 32 . Solutions of (3) give rise to the classification of Riemannian surfaces; see Corollary 9.3 , which is a nice application of the theory we develop in this paper.

The equation with various powers $\psi(u)=u^{\alpha}$ also arises in probability $(\alpha=2$ : Brownian snakes; $1<\alpha \leq 2$ : superdiffusion); see e.g. [11, 12], [19].

We consider equation (11) on a domain $\Omega$ in a Riemannian manifold $M$. The main result of this article is that both questions (the existence of a large positive solution and its uniqueness) are local properties. That is, they depend solely on the properties of $\partial \Omega$ near a point $x \in \partial \Omega$. We develop concepts of $\psi$-regularity and $\psi$-uniqueness of a boundary point $x \in \partial \Omega$ (see the definitions in section 2 ). What these notions mean is that a boundary point $x \in \partial \Omega$ has a neighborhood $U$ such that the problem $\Delta u-c \psi(u)=0$ in $\Omega \cap U$ has a large solution for some $c>0$ (or a unique large solution for $\psi$-uniqueness for some $c>0$ ). More will be discussed when these notions are properly introduced. To put our results into context, the question of existence of large solutions for functions $\psi(u)=u^{q}$ for $1<q<\infty$ has been settled in the works 24, 25, where it has been shown that the solvability is equivalent to certain Wiener-type conditions at every boundary point $y \in \partial \Omega$. Hence at least in this special case, the solvability is indeed a local property. From this perspective it is not surprising that the existence (as well as uniqueness) of large solutions is a local property for more general functions.

We present our main results in section 3 after all notions are defined. There are two subsections, the first of which is on the existence with main results Theorems 3.1 and 3.2 which imply that equation (1) is solvable if and only if every point of the boundary of $\Omega$ is $\psi$-regular. 
The second subsection on (non)uniqueness first presents two examples of nonuniqueness (Examples 3.1 and 3.2) on very general (fractal) domains. The construction is given in detail in section 7 and is a generalization of known examples in a smooth setting. The common feature of these examples is that the solutions $u(x)$ grow to infinity slowly as $x \rightarrow \partial \Omega$. In particular, the new metric which would arise in the Yamabe problem or in the two dimensional problem is not complete. If we require that $u$ grows sufficiently fast (we say $u$ has maximal rate of blowup), then such a solution (if it exists) is unique (Proposition 3.4). This type of behavior has already been noted in some cases in the works of Delanoë, Finn, McOwen and Tang.

The general results on uniqueness (Theorem 3.6 and Theorem 3.7) state that again uniqueness is a local property; i.e., the solution of equation (1) is unique if and only if all its points are $\psi$-unique. We also present several new necessary and sufficient conditions for uniqueness of the large solution in Theorem 3.8 and Proposition 9.9. These generalize the uniqueness condition from [39] and [42]. We conjecture that they can be further improved (see the conjecture just above Proposition 9.9).

We devote the last section to examples of how this theory can be applied to concrete examples of the functions $\psi$. Most results of this section are not new; the point we want to make is to show how they all fit nicely into the same framework. We show that in many cases existence and uniqueness do not depend on the metric tensor $g$ on $M$; i.e., if we know the answer in $\mathbb{R}^{n}$ we have it on any manifold $M$ (e.g. Corollary 9.2 and Proposition 9.4). These results nicely complement the recent progress in the special case of $\psi(u)=u^{\alpha}$ in $\Omega \subset \mathbb{R}^{n}$ where we now know the necessary and sufficient conditions for existence of a large solution in terms of Wiener-type capacity. Labutin's result states that the large solution exists if every point has a "local complement" that is the set $U \cap\left(\mathbb{R}^{n} \backslash \Omega\right)$ for all neighborhoods $U$ of $x$ of sufficiently large capacity. What "capacity" means depends on $\alpha>1$. The uniqueness in not considered in his work. Our approach follows a different path; we treat a much broader range of problems with less case-specific results.

Section 4, although auxiliary, contains important results about large solutions such as the comparison principle, existence of barriers and the Harnack inequality.

\section{Definitions}

2.1. Preliminary definitions. As mentioned in the introduction we consider positive solutions of the equation

$$
\Delta u+h u-k \psi(u)=-f \quad \text { in } \Omega .
$$

Definition 2.1. A positive solution of equation (4) which satisfies

$$
u(x) \rightarrow \infty \quad \text { as } \delta(x)=\operatorname{dist}(x, \partial \Omega) \rightarrow 0
$$

is called a large solution.

Definition 2.2. We say that the function $\psi$ satisfies the standard assumptions if

$$
\begin{aligned}
& \psi:[0, \infty) \rightarrow[0, \infty), \\
& \psi \text { is continuous, increasing on }[0, \infty), \\
& \psi(0)=0 \text { and } \psi \text { is strictly convex, i.e., } \\
& \psi(t x+(1-t) y)<t \psi(x)+(1-t) \psi(y) \text { for all } 0 \leq x<y \text { and } 0<t<1,
\end{aligned}
$$


and for some $a>0$,

$$
\int_{a}^{\infty}\left(\int_{0}^{s} \psi(u) d u\right)^{-1 / 2} d s<\infty .
$$

Condition (77) is a classical condition introduced by Keller and Osserman [21], 44. We note that (6) implies that $\psi(u) \leq k u$ for some $k>0$ and $u \in[0, \varepsilon)$; hence, the integral (17) converges to $\infty$ as $a \rightarrow 0+$. It is also worth pointing out that the condition (6) says something about the differentiability of $\psi$. Since $\psi$ is convex we know that $\psi^{\prime}$ exists almost everywhere. It also follows that $\psi(u) / u$ is a monotone increasing function; hence it has a one-sided limit at zero, so the right-derivative $\psi^{\prime}(0)$ is well defined and finite.

In several cases we shall in place of (7) assume a slightly stronger condition (8). Functions $\psi$ for which (8) holds will give rise to solutions that satisfy the well-known Harnack inequality (see below); hence we name this condition appropriately.

Definition 2.3. If, in addition to condition (6), there exists $\theta>1$ such that

$$
\liminf _{u \rightarrow \infty} \frac{\psi(\theta u)}{\theta \psi(u)}>1,
$$

we say that $\psi$ satisfies the Harnack assumptions.

If is easy to see that (8) implies that $\psi(u) \geq c u^{1+\varepsilon}$, where $\varepsilon=\ln y / \ln \theta>0$ and $y$ is any number between 1 and $\liminf _{u \rightarrow \infty} \psi(\theta u) /(\theta \psi(u))$. Clearly such a $\psi$ satisfies (7). To see that these conditions are not equivalent, consider $\psi$ equal to $u \ln ^{4}(1+u)$ for large $u$. We see that $\psi$ satisfies (7) but not (8), so (8) is a slightly stronger condition. All $\psi$ we will consider in examples do satisfy (8).

The condition (usually easy to verify)

$$
\liminf _{u \rightarrow \infty} \frac{u \psi^{\prime}(u)}{\psi(u)}>1
$$

is even stronger, as it implies that (8) holds for all $\theta>1$; hence $\psi$ satisfies the strong Harnack assumptions.

Definition 2.4. Let $\Omega$ be a domain in $M$. We say that two positive functions $u_{1}$, $u_{2}$ defined on $\Omega$ are comparable if

$$
0<\inf _{x \in \Omega} \frac{u_{1}(x)}{u_{2}(x)} \leq \sup _{x \in \Omega} \frac{u_{1}(x)}{u_{2}(x)}<\infty .
$$

We write $u_{1} \approx u_{2}$. If, in addition, $u_{1}, u_{2}$ are large solutions of equation (4) we say that $u_{1}, u_{2}$ are comparable large solutions.

Definition 2.5. Let $\Omega$ be a domain in $M$ and let $\varphi(x)=\psi(x) / x$. We say that a large solution $u$ of equation (44) is complete if

$$
\varphi(u(x)) \geq \frac{1}{\delta^{2}(x) f(\delta(x))},
$$

for some nondecreasing function $f: \mathbb{R}^{+} \rightarrow \mathbb{R}^{+}$such that

$$
\int_{0}^{\varepsilon} \frac{d t}{t f(t)}=\infty, \quad \text { for some } \varepsilon>0 .
$$

We say that a complete large solution $u$ of equation (4) blows up at the maximal rate if the function $f$ in (11) can be taken to be positive and constant. 
Remark 2.1. The reason we call such a solution complete is that (if it exists) the metric it defines gives rise to a complete metric for the Yamabe equation (2). Of course, this definition is much more general, but fits nicely into the whole picture.

2.2. Regular boundary points and regular domains. We recall the classical notions of regular, $L$-regular boundary point and the concept of a barrier. These notions were first introduced for the Laplacian in $\mathbb{R}^{n}$ but generalize to second-order elliptic operators on manifolds; cf. [20] and [48. Let $\Omega$ be an arbitrary domain $\Omega \subset M$ with nonempty boundary. Then the Dirichlet problem

$$
L u=(\Delta-V) u=0,\left.\quad u\right|_{\partial \Omega}=g \in C(\partial \Omega) \text { in some sense, }
$$

always has a solution (the Poisson integral) $u=\mathrm{PI}(g)$, where PI:C $(\partial \Omega) \rightarrow L^{\infty}(\Omega)$. Here $V \in L^{n / 2+\varepsilon}(\Omega)$ for some $\varepsilon>0$ and $V \geq 0$. If $V \in C^{\varepsilon}(\Omega)$, then $u \in C_{l o c}^{2}(\Omega)$.

Definition 2.6. A point $x \in \partial \Omega$ is called L-regular if for all $g \in C(\partial \Omega)$ the

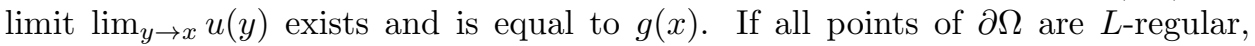
then we say that the domain $\Omega$ is $L$-regular. Obviously in such a case we have: $\mathrm{PI}: C(\partial \Omega) \rightarrow C(\bar{\Omega})$.

We say that a point $x \in \partial \Omega$ is regular if it is $L$-regular for $L=\Delta$.

All $L$-regular points can be characterized in terms of local barriers.

Theorem 2.7. The point $x \in \partial \Omega$ is $L$-regular if and only if there exists a neighborhood $U$ of $x$ and $a C^{2}$ function $w$ defined on $U \cap \Omega$, such that $L w=(\Delta-V) w \leq 0$, $w(y) \rightarrow 0$ as $y \rightarrow x$ and for any neighborhood $U^{\prime}$ of $x$ there is $\delta>0$ such that $w(y)>\delta$ for all $y \in(U \cap \Omega) \backslash U^{\prime}$.

Corollary 2.8. If $x$ is a regular point, then $x$ is $L$-regular for all $L=\Delta-V$, $V \geq 0$.

We now want to generalize the notions of $L$-regular points and domains for our purposes. In our case these notions will depend on the given function $\psi$; hence we will talk about $\psi$-regularity.

Definition 2.9. Suppose that the function $\psi$ satisfies the Harnack assumptions. Let $\Omega \subset M$ be a domain with a nonempty boundary. We say that the domain $\Omega$ is $\psi$-regular if there exists at least one $c>0$ such that the equation

$$
\Delta u-c \psi(u)=0
$$

has a large solution in $\Omega$.

We say that a point $x \in \partial \Omega$ is $\psi$-regular if there exists a neighborhood $U$ of $x$ such that $U \cap \Omega$ is $\psi$-regular.

If $\psi$ satisfies only the standard assumptions we say that the domain $\Omega$ is $\psi$ regular if the equation $\Delta u-c \psi(u)=0$ has a large solution in $\Omega$ for all $c>0$. The notion of a $\psi$-regular point remains unchanged.

In parallel to classical theory we also introduce $\psi$-barriers:

Definition 2.10. Let $\Omega$ be a domain in $M$. Assume that the function $\psi$ satisfies the standard assumptions. Let $U$ be a neighborhood of a point $x \in \partial \Omega$. We say that a positive function $w$ defined in $U \cap \Omega$ is a local $\psi$-barrier at $x$ if $w$ is a subsolution of the equation $\Delta u-\psi(u)=0$, i.e., if $\Delta w-\psi(w) \geq 0$ in the sense of distributions on $U \cap \Omega$ and

$$
\lim _{y \rightarrow x} w(y)=\infty
$$


2.3. $\psi$-uniqueness. For the classical problem $L u=0$ in $\Omega,\left.u\right|_{\partial \Omega}=g \in C(\partial \Omega)$, there is no issue with uniqueness, as the maximum principle applies. There are however several examples of nonuniqueness of large solutions for equation (4), some of which can be found in this paper. For this reason we will consider the uniqueness issue in detail here and in parallel with the previous subsection will introduce a new notion of $\psi$-uniqueness. Here we will assume the stronger Harnack assumptions on the function (8). This will allow us to consider a nonconstant $k$ in equation (1); if however $k$ is constant the standard assumptions would suffice.

Definition 2.11. Let the function $\psi$ satisfy the Harnack assumptions. Let $\Omega \subset M$ be a $\psi$-regular domain with nonempty boundary.

We say that a point $x \in \partial \Omega$ is $\psi$-unique if there exists neighborhood $U$ of $x$ such that $U \cap \Omega$ is $\psi$-regular and one of the following holds:

(a) For all $c>0$ any two large solutions $u^{1}, u^{2}$ of the equation $\Delta u-c \psi(u)=0$ in $U \cap \Omega$ satisfy:

$$
\limsup _{y \rightarrow x} \frac{u^{1}(y)}{u^{2}(y)}=1
$$

(b) For some $\theta>1, \limsup _{u \rightarrow \infty} \frac{\psi(\theta u)}{\psi(u)}<\infty$ and (8) holds for a sequence of $\theta_{n}$ with limit 1. Also for some $c>0$, the maximal large solution $u^{c}$ of the equation $\Delta u-c \psi(u)=0$ in $U \cap \Omega$ is complete and any other large solution $u$ of the same equation in $U \cap \Omega$ satisfies

$$
\limsup _{y \rightarrow x} \frac{u^{c}(y)}{u(y)}<\infty
$$

Definition 2.12. Let the function $\psi$ satisfy the Harnack assumptions. We say that the domain $\Omega$ is $\psi$-unique if for some $c>0$ the equation $\Delta u-c \psi(u)=0$ has a unique large solution in $\Omega$.

\section{MAin Results}

\subsection{Existence.}

Theorem 3.1. Let $\Omega \subset M$ be an arbitrary domain with nonempty boundary. Suppose that $\psi$ satisfies the standard assumptions. Then the domain $\Omega$ is $\psi$-regular if and only if every point $x \in \partial \Omega$ is $\psi$-regular.

Theorem 3.2. Let $\Omega \subset M$ be an arbitrary domain with nonempty boundary. Suppose that $\psi$ satisfies the standard assumptions and that $c>0$ is a real number.

(a) Let $\Omega$ be a $\psi$-regular domain. Then, given any $h, k, f \in L^{\infty}(\Omega)$ such that $f \geq 0, k \geq c>0$ there exists a large nonnegative solution $U_{\text {max }}$ of the equation

$$
\Delta u+h u-k \psi(u)=-f \quad \text { in } \Omega,
$$

with the property that any other nonnegative solution $u$ of equation (17) in $\Omega$ satisfies $u \leq U_{\max }$.

Conversely, if $\psi$ satisfies the Harnack assumptions and equation (17) has at least one large solution for some $h, k, f \in L^{\infty}(\Omega)$ such that $f \geq 0, k \geq c>0$, then the domain $\Omega$ is $\psi$-regular.

(b) If all boundary points of $\Omega$ are regular, then there also exists a minimal large solution $U_{\min }$ of equation (17), i.e., if $u$ is any other large solution of equation (17), then $u \geq U_{\min }$. 
As we will see in Examples 3.1 and 3.2 the concept of a minimal large nonnegative solution does not make sense on more general domains, i.e., domains that are not regular, since on such domains it is possible to construct a decreasing sequence of large solutions with limit zero. If $k$ is continuous at $\partial \Omega$, then we have uniqueness in (b), that is, $U_{\min }=U_{\max }$; hence there is only one large solution. We think that this is true even for discontinuous $k \in L^{\infty}$, but we do not have the proof.

Conjecture. If all boundary points of $\Omega$ are regular and $k \in L^{\infty}(\Omega), k \geq c>0$, then $U_{\min }=U_{\max }$, so uniqueness holds and the domain $\Omega$ is $\psi$-unique.

Theorem 3.3. Let $\Omega$ be a domain in $M$. Assume that the function $\psi$ satisfies the Harnack assumptions. Then $\Omega$ is a $\psi$-regular domain if and only if every point $x \in \partial \Omega$ has a local $\psi$-barrier at $x$.

If $\psi$ only satisfies the standard assumptions, then $\Omega$ is a $\psi$-regular domain if and only if every point $x \in \partial \Omega$ has a local $(c \psi)$-barrier at $x$ for every $c>0$.

All regular points have a local $(c \psi)$-barrier at $x$ for every $c>0$.

\section{2. (Non) uniqueness.}

Example 3.1. Let $\Omega \subset M$ be a domain in $M$ such that $\Gamma=M \backslash \Omega$ can be written as $\Gamma=\bigcup \Gamma_{i}(i \in I, I$ can be uncountable $)$, where each $\Gamma_{i}$ satisfies

$$
H^{n-2}(\Gamma \cap B(x, r)) \leq c r^{n-2},
$$

for all $x \in \Gamma$ and $0<r<\operatorname{diam}(M)$ and some $c$ independent of $x$ and $r$. Here $H^{n-2}$ denotes the $(n-2)$-dimensional Hausdorff measure on $M$. Also assume that for each $x \in \partial \Omega$ there is $i \in I$ such that $\limsup _{r \rightarrow 0+} r^{2-n} H^{n-2}\left(\Gamma_{i} \cap B(x, r)\right)>0$. If

$$
\limsup _{x \rightarrow \infty} \frac{\psi(x)}{\exp (k x)}=0, \quad \text { for some } k>0,
$$

then $\Omega$ is a $\psi$-regular domain. In particular, if $\operatorname{dim} M=2$, then any domain for $\psi$ satisfying (19) is $\psi$-regular.

Example 3.2. Let $\Omega \subset M$ be a domain in $M$ such that $\Gamma=M \backslash \Omega$ is Ahlfors-David regular; i.e., for some $c_{1}, c_{2}>0$ and $d<n-2$ we have that

$$
c_{1} r^{d} \leq H^{d}(\Gamma \cap B(x, r)) \leq c_{2} r^{d}, \quad \text { for all } x \in \Gamma \text { and } 0<r<1 .
$$

Then $\Omega$ is a $u^{\alpha}$-regular domain for all $1<\alpha<(n-d) /(n-d-2)$. The large solution is not unique on $\Omega$. (This claim also follows from [24] or [42], but we also get nonuniqueness.)

In particular, if $\alpha<n /(n-2)$, then any domain with nonempty boundary is $u^{\alpha}$-regular.

If $\mathcal{C} \subset[0,1]$ is the Cantor set (constructed by removing the middle third of each interval at every step), then (20) holds for $d=\ln 2 / \ln 3=0.630 \ldots$ Let $e_{1}$ be a unit vector in $\mathbb{R}^{n}$. It follows that $B(0,2) \backslash \mathcal{C} e_{1}$ is $u^{\alpha}$-regular for all $\alpha<$ $(n-\ln 2 / \ln 3) /(n-\ln 2 / \ln 3-2)$ and $n \geq 3$. The solution is not unique.

The important point of the previous examples is that the constructed solutions in section 7 go to infinity quite slowly. We do have the following uniqueness in the class of comparable solutions by an argument essentially identical to [42.

Theorem 3.4 (Uniqueness of comparable solutions). Suppose that the function $\psi$ satisfies the standard assumptions. Let $\Omega \subset M$ be a $\psi$-regular domain with nonempty boundary and $u$ a large solution of the equation $\Delta u-\psi(u)=0$ in $\Omega$. 
Assume that there exists $K>0$ such that if $v$ is another large solution of the same equation comparable to $u$, then

$$
K^{-1} \leq \inf _{x \in \Omega} \frac{u(x)}{v(x)} \leq \sup _{x \in \Omega} \frac{u(x)}{v(x)} \leq K .
$$

Then $u=v$.

An even better statement is true if the large solutions are complete:

Theorem 3.5 (Uniqueness of comparable complete solutions). Suppose that the function $\psi$ satisfies the Harnack assumptions (8) for a sequence of $\theta_{n}$ with limit 1 . Let $\Omega \subset M$ be a $\psi$-regular domain with nonempty boundary. Let $u, v$ be two large comparable complete solutions of the equation $\Delta u-\psi(u)=0$ in $\Omega$. Then $u=v$.

Theorem 3.6. Suppose that the function $\psi$ satisfies the Harnack assumptions. Then the domain $\Omega$ is $\psi$-unique if and only if all its boundary points are $\psi$-unique.

Theorem 3.7. Let $\psi$ be a function that satisfies the Harnack assumptions. Let $\Omega \subset M$ be a $\psi$-regular domain with nonempty boundary. Assume that $h, k, f \in$ $L^{\infty}(\Omega)$ are such that $f \geq 0, k \geq c_{0}$ for some $c_{0}>0$. In addition, let us assume that $k$ is a continuous function at every boundary point $\partial \Omega$. If all boundary points of $\Omega$ are $\psi$-unique, then the equation

$$
\Delta u+h u-k \psi(u)=-f, \quad \text { in } \Omega
$$

has a unique large solution.

Conversely, if for some $f, h, k \in L^{\infty}(\Omega), f \geq 0, k$ continuous at $\partial \Omega, k \geq c_{0}>0$ the equation

$$
\Delta u+h u-c k \psi(u)=-f, \quad \text { in } \Omega
$$

has a unique large solution for some $c>0$, then the domain $\Omega$ is $\psi$-unique.

Now we state one sufficient condition for $\psi$-uniqueness. This condition works only on a special class of manifolds with a rich set of local isometries, i.e., manifolds that look locally as $\mathbb{T}^{n}, \mathbb{S}^{n}$ or $\mathbb{H}^{n}$. This does not represent any restriction in two dimensions as any compact Riemannian manifold $M$ can be equipped with a conformally equivalent metric of constant curvature 1,0 or -1 , so $M$ is either $\mathbb{S}^{2}, \mathbb{T}^{2}$ or locally $\mathbb{H}^{2}$. The following proposition is an improvement of a uniqueness result from [39] which was formulated in $\mathbb{R}^{n}$ and it required that $\mathbb{R}^{n} \backslash \Omega$ has nonempty interior near every point $x \in \partial \Omega$.

Theorem 3.8. Let $\psi$ satisfy the standard assumptions and let $\Omega \subset M$ be a $\psi$ regular domain. Let $x \in \partial \Omega$ be such that there exists a neighborhood $U$ of $x$ with the following property. For every connected component $W$ of $U \cap \Omega$ there exists a continuous family of isometries $\Phi_{t}: U \rightarrow \Phi_{t}(U) \subset M, t \in[0, \varepsilon)$ such that

(a) $\Phi_{0}$ is the identity on $U$,

(b) $\overline{\Phi_{t}(W)} \subset \Omega$ for $t>0$.

Then the point $x$ is $\psi$-unique. If all points of $\Omega$ have this property, then $\Omega$ is $\psi$-unique.

Corollary 3.9. Let $\Gamma=\mathbb{T}^{n} \backslash \Omega$ be locally a graph of an $(n-1)$-dimensional surface. Then if $\Omega$ is $\psi$-regular, it is also $\psi$-unique.

A similar observation has been made in [42] (cf. Theorem 0.3 there). 
Proof of the corollary. For any $x \in \Gamma$ we identify some neighborhood $U$ of $x$ with a neighborhood $V$ of 0 in $T_{x} \mathbb{T}^{n}$ via the exponential map (this is a local isometry on $\left.\mathbb{T}^{n}\right)$. Then there is a unit vector $v \in T_{x} \mathbb{T}^{n}$ and a function $\varphi: W=V \cap\{w \in$ $\left.T_{x} \mathbb{T}^{n} ; w \perp v\right\} \rightarrow \mathbb{R}$ such that $\varphi(0)=0$ and $\Gamma \cap U=\{x+w+v \varphi(w) ; w \in W\}$.

Indeed, if this holds, then $\Gamma \cap U$ has two connected components, one "above" and one "below" the graph. Then $\Phi_{t}(y)=y+t v$ for $t>0$ is the family of isometries for the component "above" the graph and $\Phi_{t}(y)=y-t v$ for $t>0$ is the family of isometries for the component "below" the graph. Finally, we note that with a bit of an effort it is also possible to handle surfaces with corners; more can be found in Proposition 9.9 .

\section{Preliminaries: The comparison lemma, barriers AND THE HARNACK INEQUALITY}

The results in this section are mostly generalizations of known results from the case $\psi(u)=u^{\alpha}$ to a general $\psi$. For briefness we will omit certain details and concentrate on the differences. We start with a crucial comparison result.

\subsection{The comparison lemma.}

Lemma 4.1. Let $\Omega \subset M$ be a connected subdomain of $M$ with nonempty boundary. Let $h, k \in L^{n / 2+\varepsilon}(\Omega)$, for some $\varepsilon>0, k \geq 0$, and $k>0$ on a set of positive measure in $\Omega, f \in L_{-1, l o c}^{2}(\Omega), f \geq 0$ and $\psi$ satisfies (6). Let $u_{1} \in L_{1, l o c}^{2}(\Omega) \cap C(\bar{\Omega})$ be a weak positive supersolution and $u_{2} \in L_{1, l o c}^{2}(\Omega) \cap C(\bar{\Omega})$ be a weak nonnegative subsolution of (4), i.e.,

$$
\begin{aligned}
& \int_{\Omega}\left(\nabla u_{1} \nabla w-h u_{1} w+k \psi\left(u_{1}\right) w\right) d \mathrm{Vol} \geq \int_{\Omega} f w d \mathrm{Vol}, \\
& \int_{\Omega}\left(\nabla u_{2} \nabla w-h u_{2} w+k \psi\left(u_{2}\right) w\right) d \mathrm{Vol} \leq \int_{\Omega} f w d \mathrm{Vol}, \\
& \text { for all } w \in L_{1}^{2}(\Omega), w \geq 0, \operatorname{supp} w \Subset \Omega .
\end{aligned}
$$

Under these assumptions, if

$$
\limsup _{\delta(x) \rightarrow 0}\left(u_{2}-u_{1}\right)(x) \leq 0, \quad(\text { with } \delta(x)=\operatorname{dist}(x, \partial \Omega)),
$$

then $u_{1} \geq u_{2}$ in $\Omega$. If in addition there is a point $x \in \Omega$ such that $u_{1}(x)>u_{2}(x)$ and $u_{1}, u_{2} \in C_{l o c}^{2}(\Omega)$, then $u_{1}>u_{2}$ in $\Omega$.

Proof. We modify the argument contained in [5] and also in [39]. Let $\varepsilon_{1}>\varepsilon_{2}>0$ and denote $w_{i}=\left(u_{i}+\varepsilon_{i}\right)^{-1}\left(\left(u_{2}+\varepsilon_{2}\right)^{2}-\left(u_{1}+\varepsilon_{1}\right)^{2}\right)_{+}$for $i=1,2$. Here (.) + denotes the nonnegative part of the function inside the parentheses. Clearly, $w_{i} \in L_{1, l o c}^{2}(\Omega)$, $w_{i} \geq 0$ and $w_{i}$ has compact support in $\Omega$ due to (26). By (24) with $w=w_{1}$ and $w=w_{2}$ after subtraction we get

$$
\begin{aligned}
& -\int_{\Omega}\left(\nabla u_{2} \nabla w_{2}-\nabla u_{1} \nabla w_{1}\right) d \mathrm{Vol}-\int_{\Omega} f\left(w_{1}-w_{2}\right) d \mathrm{Vol} \\
& \geq \int_{\Omega} k(x)\left(\psi\left(u_{2}\right) w_{2}-\psi\left(u_{1}\right) w_{1}\right) d \mathrm{Vol}+\int_{\Omega} h(x)\left(u_{1} w_{1}-u_{2} w_{2}\right) d \mathrm{Vol} .
\end{aligned}
$$

Let $\Omega_{+}\left(\varepsilon_{1}, \varepsilon_{2}\right)=\left\{x \in \Omega ; u_{2}(x)+\varepsilon_{2}>u_{1}(x)+\varepsilon_{1}\right\}$ and note that all integrands in (27) vanish outside this set. Clearly $w_{1}>w_{2}$ in $\Omega_{+}\left(\varepsilon_{1}, \varepsilon_{2}\right)$. The first integral on 
the left hand side of (27) is equal to

$$
-\int_{\Omega_{+}\left(\varepsilon_{1}, \varepsilon_{2}\right)}\left[\left|\nabla u_{2}-\frac{u_{2}+\varepsilon_{2}}{u_{1}+\varepsilon_{1}} \nabla u_{1}\right|^{2}+\left|\nabla u_{1}-\frac{u_{1}+\varepsilon_{1}}{u_{2}+\varepsilon_{2}} \nabla u_{2}\right|^{2}\right] d \text { Vol . }
$$

From this we conclude that the left hand side is not positive. Looking at the right hand side, if we let $\varepsilon_{1} \rightarrow 0$ we conclude that its limit is

$$
\int_{\Omega_{+}(0,0)} k(x)\left(\frac{\psi\left(u_{2}\right)}{u_{2}}-\frac{\psi\left(u_{1}\right)}{u_{1}}\right)\left(u_{2}^{2}-u_{1}^{2}\right) d \operatorname{Vol} .
$$

Clearly, as $u_{2}>u_{1}$ on $\Omega_{+}(0,0)$ by (6) we see that $\left(\frac{\psi\left(u_{2}\right)}{u_{2}}-\frac{\psi\left(u_{1}\right)}{u_{1}}\right)\left(u_{2}^{2}-u_{1}^{2}\right)>0$ on $\Omega_{+}(0,0)$. It follows that the measure of the set $\left\{x \in \Omega_{+}(0,0) ; k(x)>0\right\}$ is zero; otherwise the integral (29) is positive, which contradicts the fact that the right hand side of (27) is not positive. From this we see that $\Omega \backslash \Omega_{+}(0,0) \neq \emptyset$.

Pushing the matter further we see that the limit $\varepsilon_{1} \rightarrow 0$ of (28) must be zero. As each set $\Omega_{+}\left(\varepsilon_{1}, \varepsilon_{2}\right)$ is compactly contained in $\Omega$, the continuity of $u_{1}, u_{2}$ and the fact that $u_{1}>0$ in $\Omega$ and $u_{2}>u_{1}$ in $\Omega_{+}\left(\varepsilon_{1}, \varepsilon_{2}\right)$ imply that both $u_{1} / u_{2}$ and $u_{2} / u_{1}$ are bounded away from infinity on each $\Omega_{+}\left(\varepsilon_{1}, \varepsilon_{2}\right)$.

By fixing $K=\Omega_{+}\left(\varepsilon_{1}, \varepsilon_{2}\right)$ we notice that $\Omega_{+}\left(\varepsilon_{1}, \varepsilon_{2}\right) \subset \Omega_{+}\left(\varepsilon_{1}^{\prime}, \varepsilon_{2}^{\prime}\right)$ if $0<\varepsilon_{1}^{\prime}<\varepsilon_{1}$ and some $0<\varepsilon_{2}^{\prime}<\varepsilon_{2}$. Hence for such epsilons,

$$
\begin{aligned}
0 & \leq \int_{K}\left[\left|\nabla u_{2}-\frac{u_{2}+\varepsilon_{2}^{\prime}}{u_{1}+\varepsilon_{1}^{\prime}} \nabla u_{1}\right|^{2}+\left|\nabla u_{1}-\frac{u_{1}+\varepsilon_{1}^{\prime}}{u_{2}+\varepsilon_{2}^{\prime}} \nabla u_{2}\right|^{2}\right] d \text { Vol } \\
& \leq \int_{\Omega_{+}\left(\varepsilon_{1}^{\prime}, \varepsilon_{2}^{\prime}\right)}\left[\left|\nabla u_{2}-\frac{u_{2}+\varepsilon_{2}^{\prime}}{u_{1}+\varepsilon_{1}^{\prime}} \nabla u_{1}\right|^{2}+\left|\nabla u_{1}-\frac{u_{1}+\varepsilon_{1}^{\prime}}{u_{2}+\varepsilon_{2}^{\prime}} \nabla u_{2}\right|^{2}\right] d \text { Vol }
\end{aligned}
$$

Finally, since the last integral in (30) goes to zero as $\varepsilon_{1}^{\prime} \rightarrow 0$ it follows that the middle term of (30) must also go to zero, which means that on $K$,

$$
\nabla u_{2}=\frac{u_{2}}{u_{1}} \nabla u_{1} \quad \text { and } \quad \nabla u_{1}=\frac{u_{1}}{u_{2}} \nabla u_{1} .
$$

As $K$ was any domain $\Omega_{+}\left(\varepsilon_{1}, \varepsilon_{2}\right)$, it follows that (31) holds on $\Omega_{+}(0,0)$.

Writing $u_{2}=\frac{u_{2}}{u_{1}} u_{1}$ and differentiating (31) gives us $\nabla\left(\frac{u_{2}}{u_{1}}\right) u_{1}=\nabla u_{2}-\frac{u_{2}}{u_{1}} \nabla u_{1}=$ 0 ; hence $u_{2} / u_{1}$ is constant on each connected component of $\Omega_{+}(0,0)$. From this we conclude that $\Omega_{+}(0,0)$ is empty, as otherwise $u_{1} / u_{2}=1$ at $\partial \Omega_{+}(0,0)$. However, this implies that $u_{2} / u_{1}=1$ inside $\Omega_{+}(0,0)$, which contradicts the fact that $u_{2}>u_{1}$ on the whole $\Omega_{+}(0,0)$. Thus $\Omega_{+}(0,0)$ is empty and $u_{1} \geq u_{2}$ everywhere.

In the case $u_{1}, u_{2} \in C_{l o c}^{2}(\Omega)$ and for some $x \in \Omega, u_{1}(x)>u_{2}(x)$, then $u_{1}>u_{2}$ in $\Omega$ follows from the strong (Zarremba) maximum principle.

Remark 4.1. In Lemma 4.1 the condition $k(x)>0$ on a set of positive measure is necessary. Indeed if $k \equiv 0$ on $\Omega$, then equation (4) becomes linear. The first Dirichlet eigenfunction of the Laplace-Beltrami operator is positive inside $\Omega$ and provides a counterexample to the claim of the lemma.

Lemma 4.1 implies the existence of bounded solutions to equation (44) on very general domains. We present these in the following section. 
4.2. Barriers. In this subsection we develop certain absolute estimates on solutions of equation (44). The main goal is to show that there exists a function $B: \Omega \rightarrow \mathbb{R}^{+}$such that all finite nonnegative solutions of (4) are bounded from above by $B$, provided $\psi$ satisfies certain growth assumptions. We first consider balls in $\mathbb{R}^{n}$.

Lemma 4.2. Let $R>0$ and let $\psi$ satisfy the standard assumptions (6) and (7). Then there exists a solution $u(x)=\varphi(|x|)$ in $B(0, R)=\{x ;|x|<R\} \subset \mathbb{R}^{n}$ such that

$$
\Delta u-\psi(u)=0 \quad \text { in } B(0, R), \quad u(x) \rightarrow \infty \quad \text { as } \quad x \rightarrow \partial B(0, R) .
$$

Moreover, $u(0)$ satisfies

$$
g(R) \leq u(0) \leq g(R / \sqrt{n}), \quad \text { where } g(t)=\sup _{a>0}\left\{\int_{a}^{\infty}\left(\int_{a}^{s} \psi(u) d u\right)^{-1 / 2} d s \geq t\right\} .
$$

Note that the strict convexity of $\psi$ implies that the function $g$ defined by (33) is well defined on $(0, \infty), g$ is decreasing and $g(0+)=\infty, g(\infty)=0$.

Proof. Writing (32) in polar coordinates it suffices to find a $C^{2}$ function $\varphi$ of one variable such that

$$
\varphi^{\prime \prime}(r)+\frac{n-1}{r} \varphi^{\prime}(r)-\psi(\varphi(x))=0, \quad \varphi^{\prime}(0)=0, \quad \varphi(r) \rightarrow \infty \text { as } r \rightarrow R .
$$

Following Keller [21] and Osserman [4] we rewrite (34) in the form $\frac{d}{d r}\left(r^{n-1} \frac{d}{d r} \varphi\right)=$ $r^{n-1} \psi(\varphi)$. We integrate once, using $\varphi^{\prime}(0)=0$. This yields

$$
0 \leq \varphi^{\prime}(s) \leq s^{1-n} \psi(\varphi(s)) \int_{0}^{s} r^{n-1} d r=\frac{s}{n} \psi(\varphi(s)) .
$$

Using this estimate we get $\frac{1}{n} \psi(\varphi(r)) \leq \varphi^{\prime \prime}(r) \leq \psi(\varphi(r))$, which multiplying by $\varphi^{\prime}$ and integration gives us

$$
\left(\varphi^{\prime}(r)\right)^{2}=C(r) \int_{\varphi(0)}^{\varphi(r)} \psi(s) d s, \quad \text { for some } \frac{2}{n} \leq C(r) \leq 2 .
$$

Finally, taking the reciprocal of (36) and integrating we get

$$
r=C(r) \int_{\varphi(0)}^{\varphi(r)}\left(\int_{\varphi(0)}^{s} \psi(t) d t\right)^{-1 / 2} d s, \quad \text { for some } 1 \leq C(r) \leq \sqrt{n} .
$$

It follows from (7) that for any $R>0$ there exists $\varphi_{0}=\varphi_{0}(R)>0$ for which the solution $\varphi$ with initial condition $\varphi(0)=\varphi_{0}$ blows up at $R$, i.e., $\varphi(R-)=\infty$.

Indeed, equation (34) with boundary conditions $\varphi^{\prime}(0)=0, \varphi(R)=m$ has a unique solution for any $m>0$. Denote such a solution by $\varphi_{m}$. By letting $m \rightarrow \infty$ we obtain a function $\varphi=\lim _{m \rightarrow \infty} \varphi_{m}$. We claim that $\varphi$ is also a solution to (34) and $\varphi^{\prime}(0)=0, \varphi(R-)=\infty$. Clearly, $\varphi_{m}$ is a nondecreasing sequence of functions and $\varphi_{m}$ satisfies (37) for $r=R$. Hence, by (7) it follows that $\varphi_{m}(0)$ has a finite upper bound; otherwise the right hand side of (37) would converge to zero as $m \rightarrow \infty$, which is not possible. It follows that $\varphi(0)<\infty$; hence by the well posedness of equation (34) we conclude that $\left|\varphi_{m}(r)-\varphi(r)\right| \leq C(r)\left|\varphi_{m}(0)-\varphi(0)\right|$ for all $0 \leq r<$ $R$. It follows that $\varphi$ is well defined on $[0, R)$ and $\varphi(R-)=\lim _{m \rightarrow \infty} \varphi_{m}(R)=\infty$. 
We also have an estimate on the value of $\varphi(0)=\varphi_{0}$. Indeed, using (37) for $\varphi$ and $r=R$ we obtain

$$
\frac{R}{\sqrt{n}} \leq \int_{\varphi_{0}}^{\infty}\left(\int_{\varphi_{0}}^{s} \psi(t) d t\right)^{-1 / 2} d s \leq R .
$$

It follows that $u(0)=\varphi_{0} \in\left[g(R), g\left(\frac{R}{\sqrt{n}}\right)\right]$, where $g$ is defined by (33)).

Remark 4.2. In some interesting cases the function $g$ defined is tractable. In particular, if $\psi(u)=u^{\alpha}$ for some $\alpha>1$, then $g(t) \approx t^{2 /(1-\alpha)}$. Hence we get that

$$
u(0) \approx R^{2 /(1-\alpha)} \text {. }
$$

This a priori bound is due to Aviles, Keller, Osserman and Véron.

Next, we consider similar barrier estimates on geodesic balls around points on the compact manifold $M$.

Lemma 4.3. Assume $\psi$ satisfies the standard assumptions. There exists $R_{0}>0$ such that for all $0<R \leq R_{0}$ and all points $z \in M$, the equation

$$
\Delta u-\psi(u)=0 \quad \text { in } B(z, R)
$$

has a supersolution $u$ such that $u(x) \rightarrow \infty$ as $\operatorname{dist}(x, \partial B(z, R)) \rightarrow \infty$. Here $B(z, R)$ is a geodesic ball of radius $R$; i.e., $B(z, R)=\{y \in M$; $\operatorname{dist}(x, y)<R\}$, where the distance function is the geodesic distance given by the metric tensor $g$ on $M$.

Moreover, $u(z)$ satisfies

$$
C g(R / \sqrt{2}) \leq u(z) \leq C g(R / \sqrt{2 n}),
$$

where $g$ is the function given by (33).

Proof. Pick $R_{0}>0$ sufficiently small such that for every $z \in M$ the geodesic ball $B(z, R)$ for $R \leq R_{0}$ can be equipped with local geodesic coordinates centered at $z$. In such coordinates the metric tensor simplifies and has the form $g_{i j}(z)=\delta_{i j}$ and $\nabla g_{i j}(z)=0$. Also, if $g^{i j}$ is the inverse matrix to $g_{i j}$, then $g^{i j}(z)=\delta^{i j}$ and $\nabla g^{i j}(z)=0$. Let $g=\operatorname{det}\left(g_{i j}\right)$.

In any local coordinates using the summation convention we have

$$
\Delta u=g^{-1 / 2} \partial_{j}\left(g^{j k} g^{1 / 2} \partial_{k} u\right)
$$

Let $\Delta_{0} u$ be the operator given by (42) for $g^{i j}=\delta^{i j}$. It follows that for $u: U \rightarrow \mathbb{R}$,

$$
\left|\left(\Delta-\Delta_{0}\right) u\right| \leq\left\|g^{i j}-\delta^{i j}\right\|_{L^{\infty}(U)}\left|\nabla^{2} u\right|+\left\|g^{i j}-\delta^{i j}\right\|_{L i p(U)}|\nabla u| .
$$

Hence for any $\varepsilon>0$ there is $R_{0}>0$ such that in $U=B(z, R)$, where $0<R \leq R_{0}$ we have

$$
\left|\left(\Delta-\Delta_{0}\right) u\right| \leq \varepsilon\left(\left|\nabla^{2} u\right|+|\nabla u|\right) .
$$

This is a crucial estimate that allows us to take the $v=\varphi(|x|)$ to be the solution from Lemma 4.2 for the ball $B(0, R) \subset \mathbb{R}^{n}$ to the equation

$$
\Delta v-\frac{1}{2} \psi(v)=0 \quad \text { in } B(0, R), \quad v(x) \rightarrow \infty \quad \text { as } \quad x \rightarrow \partial B(0, R) .
$$

Then $u(x)=v(\Psi(x))$ ( $\Psi$ is the chart map) is a well-defined function in $B(z, R) \subset M$ and clearly $u(x) \rightarrow \infty$ as $\operatorname{dist}(x, \partial B(z, R)) \rightarrow 0$. We claim that $u$ is a supersolution to equation (40), provided $R_{0}>0$ is small. Indeed, $u$ solves the PDE $\Delta_{0} u-\frac{1}{2} \psi(u)=$ 
0 in $B(z, R) \subset M$. Moreover, the bounds from the previous lemma such as (35) imply that $|\nabla u| \leq C\left(R_{0}\right) \psi(u)$ and $\left|\nabla^{2} u\right| \leq C\left(R_{0}\right) \psi(u)$. It follows that

$$
\left|\left(\Delta_{0}-\Delta\right) u\right| \leq 2 \varepsilon C\left(R_{0}\right) \psi(u) \quad \text { in } B(z, R)
$$

If we pick $R_{0}>0$ sufficiently small, it follows that the right hand side of (46) can be bounded by $\frac{1}{2} \psi(u)$. Thus,

$$
\Delta u-\psi(u)=\left(\Delta_{0} u-\frac{1}{2} \psi(u)\right)+\left[\left(\Delta-\Delta_{0}\right) u-\frac{1}{2} \psi(u)\right] \leq 0 .
$$

Hence $u$ is the supersolution to (40). The estimates for $u(z)$ follow from (33).

Corollary 4.4. Let $\Omega \subset M$ be a domain with nonempty boundary. Let $h, k, f$ be $L^{\infty}(\Omega)$ functions such that $f \geq 0$ and $k \geq c$ for some $c>0$. In addition, let $\psi$ be a function satisfying (6) and (17). Then there exists a continuous function $B: \Omega \rightarrow(0, \infty)$ called $a$ barrier such that any nonnegative solution $u$ to the PDE

$$
\Delta u+h u-k \psi(u)=f \quad \text { in } \Omega, \quad u \in C_{l o c}(\Omega)
$$

is bounded by B, i.e.,

$$
0 \leq u(x) \leq B(x)
$$

Moreover, the barrier function $B$ can be taken in the form $B(x)=\varphi(\delta(x))$, for $\delta(x)=\operatorname{dist}(x, \partial \Omega)$ and some continuous nonincreasing function $\varphi:(0, \infty) \rightarrow(0, \infty)$ such that $\varphi(0+)=\infty$. Hence

$$
0 \leq u(x) \leq \varphi(\delta(x))
$$

Proof. Find $m>0$ such that $\psi(m) \geq \frac{2}{c}\left(\|f\|_{L^{\infty}(\Omega)}+\|h\|_{L^{\infty}(\Omega)} m\right)$. Next, find $R_{0}>0$ as in Lemma 4.3 such that for all $0<R \leq R_{0}$ and $z \in M$ there exists a supersolution $U$ to equation (40) satisfying (41) and $U(x) \rightarrow \infty$ as $\delta(x) \rightarrow \partial B(z, R)$. By possibly making $R_{0}$ smaller, if necessary, we can arrange that any such $U$ also satisfies $U \geq m$ thanks to (41). First, we define the barrier $B$ on the set $\left\{x \in \Omega ; \delta(x) \leq R_{0}\right\}$ by

$$
B(x)=g\left(\delta(x) \sqrt{\frac{c}{4 n}}\right),
$$

where $g$ is given by (33). Indeed, let $x \in \Omega$ such that $\delta(x) \leq R_{0}$. Let $U_{R}$ be the supersolution from Lemma 4.3 for the equation $\Delta u-\frac{c}{2} \psi(u)=0$ in $B(x, R)$. It follows from (41) that

$$
m \leq U_{R}(x) \leq g\left(R \sqrt{\frac{c}{4 n}}\right) .
$$

We compare $u$ and $U_{R}$ in the ball $B(x, R)$ using Lemma 4.1. Clearly $u$ is a bounded nonnegative solution of (48) in $B(x, R)$ and $U_{R}$ a supersolution of the same equation in $B(x, R)$ since

$$
\begin{aligned}
& \Delta U_{R}+h U_{R}-k \psi\left(U_{R}\right)+f \\
= & \left(\Delta U_{R}-\frac{c}{2} \psi\left(U_{R}\right)\right)+\left(f+h U_{R}-\frac{c}{2} \psi\left(U_{R}\right)\right)+\left(c \psi\left(U_{R}\right)-k\left(U_{R}\right)\right) \\
\leq & 0+0+0=0 .
\end{aligned}
$$

Also, since $\left(U_{R}-u\right)(x) \rightarrow \infty$ as $x \rightarrow \partial B(x, R)$ it follows that all assumptions of Lemma 4.1 are satisfied and $u \leq U_{R}$ is $B(x, R)$. Hence by (52) we see that $u(x) \leq g\left(R \sqrt{\frac{c}{4 n}}\right)$ for all $R<\delta(x)$. Taking the limit $R \rightarrow \delta(x)$ - we get that $u(x) \leq B(x)$ for $B(x)$ given by (51). 
For all $x \in \Omega$ such that $\operatorname{dist}(x, \partial \Omega)>R_{0}$ we take $B(x)=g\left(R_{0} \sqrt{\frac{c}{4 n}}\right)$. Clearly, such a $B$ can be written in the form $\varphi(\delta(x))$, as $R_{0} / \delta(x) \leq 1$.

Now, if we use Lemma 4.1 on the domain $\Omega^{\prime}=\left\{x \in \Omega, \delta(x)>R_{0}\right\}$ we see that on the boundary $\partial \Omega^{\prime}$ we have $0 \leq u(x) \leq m, m \leq B(x)$ and $u$ is a subsolution and $B$ a supersolution in $\Omega^{\prime}$. It follows that $u \leq B$ in $\Omega^{\prime}$. This concludes the proof of our corollary.

Remark 4.3. As a bonus we actually obtain in the special case $\psi(u)=u^{\alpha}$ a well-known explicit formula for $B$. Remark 4.2 gives us that there exists $C=$ $C(M, k, h, f)>0$ such that for any domain $\Omega$ we can take $B$ to be equal to

$$
B(x)=\frac{C}{\delta(x)^{2 /(\alpha-1)}} \quad \text { for all } \quad x \in \Omega .
$$

4.3. Harnack inequality and other elliptic estimates for solutions. In this subsection we prove certain general estimates that our solutions satisfy. These estimates will be useful for the question of uniqueness we deal with in the following section. We note that most results we present here are based on the work for $\psi(u)=u^{\alpha}$ in [18]. Our results are a minor modification of their argument for more general $\psi$.

Lemma 4.5 (Harnack inequality). Let $\Omega \subset M$ be an arbitrary domain. Assume that $\psi$ satisfies the Harnack assumptions. Let $u$ be a positive solution of $\Delta u-\psi(u)=$ 0 in $\Omega$. Then there exists a constant $C_{0}$ independent of $u$ and $x \in \Omega$ such that

$$
\sup _{y \in B(x, \delta / 8)} u(y) \leq C_{0} \inf _{y \in B(x, \delta / 8)} u(y),
$$

where $\delta=\operatorname{dist}(x, \partial \Omega)>0$ and $B(x, r)$ denotes an open ball of radius $r$ around $x$.

Proof. To simplify notation, let $\varphi(x)=\psi(x) / x$. Write our equation as $(\Delta-$ $V(x)) u=0$, where $V(x)=\varphi(u(x))=\psi(u(x)) / u(x)$. Then the Harnack principle for the linear equation implies that

$$
\sup _{y \in B(x, \delta / 8)} u(y) \leq C \inf _{y \in B(x, \delta / 8)} u(y),
$$

where $C>0$ depends on $n$, the ellipticity modulus of $\Delta$ and $\nu \delta$ where

$$
\nu^{2}=\sup _{y \in B(x, \delta / 2)} V(y) .
$$

We claim that $\nu \delta \leq K$ for some $K$ independent of $u$ and $x$. Having that it follows that $C$ in (56) can be taken independent of $\nu \delta$; hence (55) follows.

To estimate (57) we first develop estimates on the barrier function (51), provided $\psi(u)$ satisfies (8). Recall the definition of the function $g$ in (33). Since $\psi$ is convex we get that

$$
\begin{aligned}
\int_{a}^{s} \psi(u) d u & =\frac{1}{2} \int_{a}^{s}(\psi(u)+\psi(s+a-u)) d u \geq \int_{a}^{s} \psi((s+a) / 2) d u \\
& =(s-a) \psi((s+a) / 2)=\frac{1}{2}\left(s^{2}-a^{2}\right) \varphi((s+a) / 2) .
\end{aligned}
$$

Hence

$$
\begin{aligned}
\int_{a}^{\infty}\left(\int_{a}^{s} \psi(u) d u\right)^{-1 / 2} d s & \leq \sqrt{2} \int_{a}^{\infty} \frac{d s}{\left(s^{2}-a^{2}\right)^{1 / 2} \varphi((s+a) / 2)^{1 / 2}} \\
& \leq \sqrt{2} \sum_{n=0}^{\infty} \int_{a \beta^{n}}^{a \beta^{n+1}} \frac{d s}{\left(s^{2}-a^{2}\right)^{1 / 2} \varphi((s+a) / 2)^{1 / 2}}
\end{aligned}
$$


Here we choose $\beta$ such that $(1+\beta) / 2=\theta$. We further estimate (59) as follows. As $\varphi$ is increasing, $1 / \varphi((a+s) / 2)$ on the interval $\left[a \beta^{n}, a \beta^{n+1}\right]$ can be estimated from above by $1 / \varphi\left(a\left(1+\beta^{n}\right) / 2\right)$. Notice that $\theta^{n}=((1+\beta) / 2)^{n} \leq\left(1+\beta^{n}\right) / 2$; hence $1 / \varphi\left(a\left(1+\beta^{n}\right) / 2\right) \leq 1 / \varphi\left(a \theta^{n}\right)$. Furthermore, by (8) it follows that for sufficiently large $a$ there is $0<\tau<1$ such that $1 / \varphi\left(a \theta^{n}\right) \leq \tau^{n} / \varphi(a)$. We put all of this into (59), evaluate the integral for $n=0$ separately (we get that it is integrable) and for the rest since $\left(s^{2}-a^{2}\right)^{1 / 2} \approx s$ as $s \gg a$ we replace this term by $s$. This yields

$$
\int_{a}^{\infty}\left(\int_{a}^{s} \psi(u) d u\right)^{-1 / 2} d s \leq \frac{C}{\varphi(a)^{1 / 2}} \sum_{n=0}^{\infty} \tau^{n / 2} \ln \beta=\frac{C}{\varphi(a)^{1 / 2}} .
$$

The last estimate follows from the fact that the series is summable. It follows from the definition of the function $g$ that $g\left(C / \varphi(a)^{1 / 2}\right) \leq a$ for sufficiently large $a$ or equivalently $g(x) \leq \varphi^{-o}\left(C^{2} / x^{2}\right)$ for sufficiently small $x$. Here $\varphi^{-o}$ denotes the inverse function of $\varphi$ (to distinguish it from $\varphi^{-1}=1 / \varphi$ ).

Combining (57), (49) and (51) we get that

$$
\nu^{2} \delta^{2} \leq \varphi\left(g\left(c_{0} \delta\right)\right) \delta^{2} \leq \varphi\left(\varphi^{-o}\left(\frac{C^{2}}{c_{0} \delta^{2}}\right)\right) \delta^{2}=\frac{C^{2}}{c_{0}^{2}}
$$

i.e., $\nu \delta$ is bounded uniformly from above. From this our claim follows.

Corollary 4.6. Let $\psi$ be as in the lemma above and $\varphi(x)=\psi(x) / x$. It follows that the barrier function in Corollary 4.4 can be taken to be of the form

$$
0 \leq u(x) \leq \varphi^{-o}\left(K \delta^{-2}\right)
$$

for some $K>0$ small. Notice that in the case $\psi(u)=u^{\alpha}$ this again yields (54). However, (62) is not always optimal, e.g., when $\psi(u)=e^{u}-1$, which is another important case. In this case the definition of $g$ yields the estimate $0 \leq u(x) \leq$ $\psi^{-o}\left(K \delta^{-2}\right)$, which is better as $\varphi \ll \psi$ for $u \rightarrow \infty$.

The Harnack inequality is the crucial step in proving weighted global estimates for the solution. As the details are done in [18] we just state the results. As before let $\delta(x)=\operatorname{dist}(x, \partial \Omega)$. By $\|u\|_{2, \alpha, \Omega}$ we denote the following weighted norm:

$$
\begin{aligned}
\|u\|_{2, \alpha, \Omega} & =\sup _{x \in \Omega}|u(x)|+\sup _{x \in \Omega} \delta(x)|\nabla u(x)|+\sup _{x \in \Omega} \delta(x)^{2}\left|\nabla^{2} u(x)\right| \\
& +\sup _{x, y \in \Omega} \min \{\delta(x), \delta(y)\}^{2+\alpha} \frac{\left|\nabla^{2} u(x)-\nabla^{2} u(y)\right|}{\operatorname{dist}(x, y)^{\alpha}} .
\end{aligned}
$$

Theorem 4.7. Assume that $\psi$ satisfies the Harnack assumptions. Then there exists $C>0$ and $\alpha>0$ such that any positive solution $u$ of $\Delta u-\psi(u)=0$ in $\Omega$ satisfies

$$
\begin{aligned}
\|u\|_{2, \alpha, \Omega} & \leq C\|u\|_{L^{\infty}(\Omega)} \\
\delta(x)|\nabla u(x)|+\delta^{2}(x)\left|\nabla^{2} u(x)\right| & \leq C u(x), \quad \text { for all } x \in \Omega .
\end{aligned}
$$

Having (64) we now consider the following situation. Let $u$ be a solution of the equation $\Delta u-\psi(u)=0$ in $\Omega$. Given $\delta>0$ let $x$ be a point in $\Omega$ such that $\delta(x)=\operatorname{dist}(x, \partial \Omega)=2 \delta$ and let $B=B(x, \delta)$ be a geodesic ball of radius $\delta$ around $x$. It follows that any point of $\bar{B}$ has distance at least $\delta$ to the boundary $\partial \Omega$.

Given any $R>1$ it is obvious that $R u$ is a supersolution of the equation $\Delta u-$ $\psi(u)=0$ in $\Omega$ and therefore in $B$ as well. We ask the following question. Is it possible to find a "better" supersolution in $B$ ? That is, could we find a radial 
function $\Theta=\Theta(r)$ such that $\Theta u$ is a supersolution in $B$, and $\Theta(r)<R$ on $[0, \delta)$, $\Theta(\delta)=R$ ? The following lemma gives us the answer. This lemma represents a crucial step in the proof of uniqueness of large solutions with sufficiently fast blowup.

Lemma 4.8. Assume that $\psi$ satisfies (6) and (8) for some $\theta>1$. Let $u$ be a large solution of the equation $\Delta u-\psi(u)=0$ in a domain $\Omega$. Then there exists $\delta_{0}>0$ and $C>0$ such for all $x \in \Omega$ with $\delta(x)=2 \delta<2 \delta_{0}$ and $R>\theta$ we have that $\Theta u$ is a supersolution of the equation $\Delta u-\psi(u)=0$ in $B=B(x, \delta)$. Here, $\Theta$ is a radial function in $B$, i.e., $\Theta(z)=\Theta(\operatorname{dist}(z, x))$, and

$$
\Theta(r)=R-C\left(\inf _{z \in B} \varphi(u(z))\right)\left(\delta^{2}-r^{2}\right), \quad \text { for } 0 \leq r \leq \delta
$$

provided $\Theta(0) \geq \theta$ and $\varphi(x)=\psi(x) / x$. If this does not happen we take

$$
\Theta(r)=R-\frac{R-\theta}{\delta^{2}}\left(\delta^{2}-r^{2}\right), \quad \text { for } 0 \leq r \leq \delta .
$$

Proof. Computing $\Delta(\Theta u)$ for a radial function $\Theta$ we see that

$$
\Delta(\Theta u)=\Theta \Delta u+u \Delta \Theta+B(\nabla u, \nabla \Theta),
$$

where $B$ is a bilinear function in $\nabla u$ and $\nabla \Theta$. Hence, by (64) there is a constant $K>0$ such that

$$
|B(\nabla u(x), \nabla \Theta(r))| \leq K \Theta^{\prime}(r) \frac{u(x)}{\delta(x)},
$$

as $\Theta^{\prime}(r) \geq 0$. The constant $K$ is independent of $x$. Having (65) and (66) we see that $\Theta(r) \geq \theta$. By (8) we have that for large $u$ : $\varphi\left(\theta_{0} u\right) / \varphi(u) \geq c>1$ for any $\theta_{0} \geq \theta$. This is equivalent to $\theta_{0} \psi(u) \leq \frac{1}{c} \psi\left(\theta_{0} u\right)$. From this,

$$
\Delta(\Theta u)=\Theta \psi(u)+u \Delta \Theta+B(\nabla u, \nabla \Theta) \leq \frac{1}{c} \psi(\Theta u)+u \Delta \Theta+B(\nabla u, \nabla \Theta) .
$$

Hence

$$
\Delta(\Theta u)-\psi(\Theta u) \leq-\left(1-\frac{1}{c}\right) \psi(\Theta u)+u \Delta \Theta+B(\nabla u, \nabla \Theta) .
$$

It follows that if we find $\Theta$ such that

$$
u \Delta \Theta+B(\nabla u, \nabla \Theta) \leq\left(1-\frac{1}{c}\right) \psi(u),
$$

then since $\psi(u) \leq \psi(\Theta u)$ it follows that the right hand side of (70) is less than 0 , so $\Theta u$ is a supersolution. Next, instead of solving (71) we look for $\Theta$ such that

$$
u \Delta_{0} \Theta+B(\nabla u, \nabla \Theta) \leq \frac{1}{2}\left(1-\frac{1}{c}\right) u\left(\inf _{x \in B} \varphi(u(x))\right),
$$

where $\Delta_{0}$ is a flat Laplacian in geodesic local coordinates centered at $x$. Since (44) holds, if $\delta_{0}>0$ is chosen sufficiently small, we get that (71) follows from (72). Writing $\Delta_{0}$ in polar coordinates we get

$$
\Theta^{\prime \prime}(r)+\frac{n-1}{r} \Theta^{\prime}(t)+\frac{1}{u} B(\nabla u, \nabla \Theta) \leq \frac{1}{2}\left(1-\frac{1}{c}\right)\left(\inf _{x \in B} \varphi(u(x))\right) .
$$

Finally we note that the term $\frac{1}{u} B(\nabla u, \nabla \Theta)$ by (68) can be dominated by $\frac{K}{r} \Theta^{\prime}(t)$, since $\frac{1}{\delta(x)} \leq \frac{1}{r}$. Hence (73) will be satisfied if $\Theta$ solves

$$
\Theta^{\prime \prime}(r)+\frac{K+n-1}{r} \Theta^{\prime}(t)=\frac{1}{2}\left(1-\frac{1}{c}\right)\left(\inf _{x \in B} \varphi(u(x))\right) .
$$

It is a simple exercise that the function $\Theta$ given by (65) for some $C>0$ depending only on $K, c$ and $n$ solves this equation. We get (66) if $\Theta(0)$ given by (65) is less than $\theta$ (if we allow this, (69) no longer holds), so we make this modification. 


\section{Existence And uniqueness for CONTINuOus DiRichlet DATA}

In this section we establish the existence and uniqueness of large solutions in domains with relatively nice boundary. In section 2 we defined a regular point $x \in \partial \Omega$. In such a point the Poisson integral for the Laplace equation in $\Omega$ with $C(\partial \Omega)$ data gives a continuous function at $x$. We will consider for now domains $\Omega$ with all boundary points regular. The following theorem (cf. [48]) gives sufficient (but not necessary) conditions for a point to be regular.

Theorem 5.1. The point $x \in \partial \Omega$ is regular if there exists a neighborhood $U$ of $x$ and smooth coordinates on $U$ in which there exists a cone $C$ with vertex $x$ such that $U \cap C \backslash\{x\} \subset M \backslash \bar{\Omega}$.

In particular, all boundary points of a Lipschitz domain are regular.

Now we tackle equation (4). We first settle the question of the existence and uniqueness of a solution to the Dirichlet problem with continuous boundary data under the additional assumption $h \geq 0$. In this case we want to consider all solutions, not only the positive one. To accommodate this extension we have to extend the domain of $\psi$ onto the whole $\mathbb{R}$.

Theorem 5.2. Let $\Omega \subset M$ be a domain with nonempty boundary such that all boundary points are regular. Also assume that $h, k \geq 0, h, k \in L^{n / 2+\varepsilon}(\Omega)$ for some $\varepsilon>0$, and $\psi: \mathbb{R} \rightarrow \mathbb{R}$ satisfies ([6) for both $\psi_{1}(x)=\left.\psi(x)\right|_{[0, \infty)}$ and $\psi_{2}(x)=$ $-\left.\psi(-x)\right|_{[0, \infty)}$. Moreover, assume that

$$
\inf _{x>0} \frac{\psi_{1}(x)}{x}=\inf _{x>0} \frac{\psi_{2}(x)}{x} .
$$

Then for any $g \in C(\partial \Omega)$ and a distribution $f=\Delta F$ for some $F \in C(\bar{\Omega})$ the Dirichlet problem

$$
\Delta u-h u-k \psi(u)=f \quad \text { in } \Omega, \quad u \in C(\bar{\Omega}),\left.\quad u\right|_{\partial \Omega}=g
$$

has a unique solution. There exists $C>0$ such that all solutions to (76) satisfy the estimate

$$
\|u\|_{C(\Omega)} \leq C\left(\|F\|_{C(\Omega)}+\|g\|_{C(\partial \Omega)}\right) .
$$

If in addition $f \leq 0$ and $g \geq 0$ the solution $u$ is nonnegative.

Proof. Consider the linear problem

$$
(\Delta-V) u=f \quad \text { in } \Omega, \quad u \in C(\bar{\Omega}),\left.\quad u\right|_{\partial \Omega}=g,
$$

for arbitrary $V \in L^{n / 2+\varepsilon}(\Omega), V \geq 0$. We want to establish the existence of a solution for this problem. Clearly, if $f=0$ there is nothing to prove, since (78) is just the same equation as (13) and hence Theorem 2.7 and Corollary 2.8 apply.

If $f \neq 0$, let $\widetilde{F}$ be a continuous extension of $F$ on the whole $M$ and let $\widetilde{f}=\Delta \widetilde{f}$. Since we can always arrange that $V>0$ on a set of positive measure on each connected component of $M \backslash \Omega$ it follows (cf. [35]-[38, [7]-9]) that $L=\Delta-V$ is invertible on $M$. Consider

$$
w=(\Delta-V)^{-1} \widetilde{f}=(\Delta-V)^{-1} \Delta \widetilde{F}=\widetilde{F}+(\Delta-V)^{-1}(V \widetilde{F}) .
$$

As $(V \widetilde{F}) \in L^{n / 2+\varepsilon}(M)$, the mapping properties of $(\Delta-V)^{-1}$ imply that $(\Delta-$ $V)^{-1}(V \widetilde{F}) \in C^{\alpha}(\bar{\Omega})$ for some $\alpha>0$. It follows that $w \in C(M)$. Hence $u=v+w$ 
is a solution to (78), provided that $v$ solves

$$
(\Delta-V) v=0 \quad \text { in } \Omega, \quad v \in C(\bar{\Omega}),\left.\quad v\right|_{\partial \Omega}=g-\left.w\right|_{\partial \Omega} .
$$

This establishes the existence. For uniqueness, see e.g. [8]. Moreover, there exists $C>0$ independent of $V$ such that the solution $u$ to (178) satisfies the estimate

$$
\|u\|_{C(\Omega)} \leq C\left(\|F\|_{C(\Omega)}+\|g\|_{C(\partial \Omega)}\right) .
$$

The proof of (81) can be split into two cases. The first case occurs when $f=0$ and $g \neq 0$, and the estimate follows directly from the maximum principle, as $|u(x)| \leq \sup _{y \in \partial \Omega}|u(y)|$. The second case $g=0$ and $f \neq 0$ follows from an intriguing lemma established in [10] (cf. Lemma 2.7). Even though this lemma is formulated for Lipschitz domains, it follows from the proof that the $L^{\infty}$ version works on any domain. Moreover, the computation there gives the precise value of $C$, namely $C=2$. Combining these two cases we have that (81) holds; in fact, the stronger estimate

$$
\|u\|_{C(\Omega)} \leq 2\|F\|_{C(\Omega)}+\|g\|_{C(\partial \Omega)}
$$

is also true.

Equipped with this estimate we take on (176). The properties of function $\psi$ imply that a new function defined by $\Psi(u)=\psi(u) / u$ if $u \neq 0$ and $\Psi(0)=\inf _{u \neq 0} \psi(u) / u=$ $\lim _{u \rightarrow 0} \psi(u) / u$ is nonnegative on $\mathbb{R}$ and continuous.

We fix $f$ and $g$ as in the statement of our theorem and consider the number $M=2\|F\|_{C(\Omega)}+\|g\|_{C(\partial \Omega)}$. Then let $T u=v$ be a map $T: C(\bar{\Omega}) \rightarrow C(\bar{\Omega})$ defined by the PDE

$$
(\Delta-(h+k \Psi(u))) v=f \quad \text { in } \Omega, \quad v \in C(\bar{\Omega}),\left.\quad v\right|_{\partial \Omega}=g .
$$

Let $V(x)=h(x)+k(x) \Psi(u(x))$. It follows that $V \geq 0$ and $V \in L^{n / 2+\varepsilon}(\Omega)$, since $h, k \in L^{n / 2+\varepsilon}(\Omega)$ and $\Psi(u) \in L^{\infty}(\Omega)$ for $u \in C(\bar{\Omega})$. Hence $V$ is as in (178), so the solution $v$ to (83) exists and satisfies the estimate (82), i.e., $\|v\|_{C(\bar{\Omega})} \leq M$ for all $u \in C(\bar{\Omega})$. Hence $T$ maps the ball

$$
B_{M}=\left\{u \in C(\bar{\Omega}):\|u\|_{C(\bar{\Omega})} \leq M\right\}
$$

into itself. We prove that $T$ is continuous and compact; hence by the Schauder fixed point theorem, $T$ has a fixed point. Clearly, the fixed point of $T$ solves (76).

Let us first deal with the issue of continuity of $T$. Let $u_{n}$ be a sequence from $B_{M}$ converging to some $u \in B_{M}$ in the $C(\bar{\Omega})$ norm. Since $\Phi$ is continuous on $\mathbb{R}$ it is uniformly continuous on the interval $[-M, M]$. It follows that $\Phi\left(u_{n}\right) \rightarrow$ $\Phi(u)$ in $C(\bar{\Omega})$ as all $\left|u_{n}(x)\right| \leq M$ for all $x \in \bar{\Omega}$. It follows that $V_{n}(x)=h(x)+$ $k(x) \Psi\left(u_{n}(x)\right) \rightarrow V(x)=h(x)+k(x) \Psi(u(x))$ in $L^{n / 2+\varepsilon}(\Omega)$ for some $\varepsilon>0$. Now by looking at the difference $w_{n}=v-v_{n}$, where $v_{n}=T\left(u_{n}\right)$ and $v=T(u)$, we see that $w_{n}$ is a solution to the $\mathrm{PDE}$

$$
(\Delta-V) w_{n}=f_{n}=\left(V-V_{n}\right) u_{n}, \quad w_{n} \in C(\bar{\Omega}),\left.\quad w_{n}\right|_{\partial \Omega}=0 .
$$

It follows that $\left\|f_{n}\right\|_{L^{n / 2+\varepsilon}(\Omega)} \leq\left\|V-V_{n}\right\|_{L^{n / 2+\varepsilon}(\Omega)}\left\|u_{n}\right\|_{C(\bar{\Omega})} \rightarrow 0$ as $n \rightarrow \infty$. Then in the light of the estimate (82) and the fact that for each $f_{n}$ there is $F_{n}$ such that $\Delta F_{n}=f_{n}$ with $\left\|F_{n}\right\|_{C(\bar{\Omega})} \leq C\left\|f_{n}\right\|_{L^{n / 2+\varepsilon}(\Omega)}$ we see that

$$
\left\|w_{n}\right\|_{C(\bar{\Omega})} \leq C\left\|f_{n}\right\|_{L^{n / 2+\varepsilon}(\Omega)} \rightarrow 0, \quad \text { as } n \rightarrow \infty .
$$


This proves continuity. Now we show compactness. Take an arbitrary sequence $\left(u_{n}\right)_{n \in \mathbb{N}}$ of points from $B_{M}$. Each $v_{n}=T\left(u_{n}\right)$ is also in $B_{N}$; hence the $L^{n / 2+\varepsilon}(\Omega)$ norm of $f_{n}=\left(h+k \Phi\left(u_{n}\right)\right) v_{n}$ can be bounded by a constant independent of $n$. It follows that $v_{n}$ solves the PDE

$$
\Delta v_{n}=f_{n}, \quad v_{n} \in C(\bar{\Omega}),\left.\quad v_{n}\right|_{\partial \Omega}=g .
$$

We claim that this implies that $\left(v_{n}\right)_{n \in \mathbb{N}}$ has a convergent subsequence in $C(\bar{\Omega})$. Indeed, consider the extension of $f_{n}$ onto $M$ by putting $\widetilde{f_{n}}=f_{n}$ on $\bar{\Omega}$ and $\widetilde{f_{n}}=0$ on $M \backslash \bar{\Omega}$. The Laplace-Beltrami operator $\Delta$ is not invertible on functions on $M$, since all constants are annihilated by $\Delta$, but the operator $\Delta-V$ for $V=0$ in $\Omega$, $V=1$ on the set $M \backslash \Omega$ is. Moreover, the mapping properties of this operator (cf. [35]) imply that $\widetilde{w_{n}}=(\Delta-V)^{-1} \widetilde{f_{n}} \in C^{\alpha}(M)$ for some $\alpha>0$ and $\left\|\widetilde{w_{n}}\right\|_{C^{\alpha}(M)} \leq$ $C\left\|\widetilde{f_{n}}\right\|_{L^{n / 2+\varepsilon}(\Omega)} \leq C$. Let $w_{n}=\left.\widetilde{w_{n}}\right|_{\bar{\Omega}}$. Clearly, $\Delta w_{n}=f_{n}$ in $\Omega$. Now as the Hölder space $C^{\alpha}(\bar{\Omega})$ is compact in $C(\bar{\Omega})$, it follows that there is a subsequence of $w_{n}$ convergent in $C(\bar{\Omega})$. Slightly abusing the notation, we will refer to this subsequence as $\left(w_{n}\right)_{n \in \mathbb{N}}$. Denote the limit of $w_{n}$ in $C(\bar{\Omega})$ by $w_{0}$. Finally, let $V_{n}$ for $n=0,1,2,3, \ldots$ be the solution of the PDE

$$
\Delta V_{n}=0, \quad V_{n} \in C(\bar{\Omega}),\left.\quad V_{n}\right|_{\partial \Omega}=g-\left.w_{n}\right|_{\partial \Omega} .
$$

We see that $v_{n}=V_{n}+w_{n}$ and since $g-\left.w_{n}\right|_{\partial \Omega} \rightarrow g-\left.w_{0}\right|_{\partial \Omega}$ in $C(\partial \Omega)$ the estimate (82) implies that $V_{n} \rightarrow V_{0}$ in $C(\bar{\Omega})$. Putting things together this implies that $v_{n} \rightarrow v_{0}$ in $C(\bar{\Omega})$; hence the map $T$ is compact. This finishes the proof of existence of a solution to (76).

The uniqueness is trivial. Assume there are two solutions $u_{1}$ and $u_{2}$ in $C(\bar{\Omega})$. Then their difference $w=u_{1}-u_{2}$ solves the PDE

$$
\left(\Delta-V_{12}\right) w=0, \quad w \in C(\bar{\Omega}),\left.\quad w\right|_{\partial \Omega}=0
$$

where

$$
V_{12}(x)=h(x)+k(x)\left(\frac{\psi\left(u_{1}(x)\right)-\psi\left(u_{2}(x)\right)}{u_{1}(x)-u_{2}(x)}\right) \quad \text { for } x \text { such that } u_{1}(x) \neq u_{2}(x) .
$$

We take $V_{12}(x)=h(x)$ if $u_{1}(x)=u_{2}(x)$. Clearly $V_{12} \geq 0$ as $\psi$ is an increasing function. Also since $u_{1}, u_{2} \in[-M, M]$ for some $M$ large enough, the quotient

$$
\frac{\psi\left(u_{1}(x)\right)-\psi\left(u_{2}(x)\right)}{u_{1}(x)-u_{2}(x)} \leq \max \{\psi(M+1)-\psi(M), \psi(M)-\psi(M-1)\} .
$$

It follows that $V_{12} \in L^{n / 2+\varepsilon}(\Omega)$. Thus, using the estimate (82) one more time equation (89) implies that $w \equiv 0$. From this uniqueness follows.

Finally, the last claim that $u \geq 0$ when $f \leq 0$ and $g \geq 0$ is an easy consequence of the maximum principle.

Next we deal with the general case. As we can see, our claim in this case is weaker than in the most favorable case contained in Theorem 5.2. We also consider only the nonnegative solutions.

Theorem 5.3. Let $\Omega \subset M$ be a domain with nonempty boundary such that all boundary points are regular. Also assume that $k \geq 0, h, k \in L^{n / 2+\varepsilon}(\Omega)$ for some 
$\varepsilon>0$ and that $\psi$ satisfies (6). Let $f=\Delta F$ for some $F \in C(\bar{\Omega})$ and $f \leq 0$ in the sense of distributions. Assume that the equation

$$
\Delta u-h u-k \psi(u)=f \quad \text { in } \Omega, \quad u \in C(\bar{\Omega}),
$$

has at least one positive supersolution $u$ in $C(\bar{\Omega})$, i.e., $\Delta u-h u-k \psi(u) \leq f$ in the sense of distributions.

Then for any $g \in C(\partial \Omega), g \geq 0$ there exists at least one nonnegative solution $u$ of the equation (91) satisfying $\left.u\right|_{\partial \Omega}=g$.

In addition, if $k>0$ in $\Omega, f \in L_{-1, l o c}^{2}(\Omega)$, and either $f$ or $g$ does not vanish, then the solution is also unique. The uniqueness also follows if $k>0$ on a set of positive measure, $k, h, f \in C_{\text {loc }}^{\alpha}(\Omega)$ for some $\alpha>0$ and either $f$ or $g$ does not vanish.

Proof. Let us denote the positive supersolution of equation (91) by $U_{\max }$. Without loss of generality we can assume that at the boundary $\partial \Omega$ we have $\left.U_{\max }\right|_{\partial \Omega} \geq g$. Indeed, if this does not hold, then since $\inf _{x \in \partial \Omega} U_{\max }(x)>0$ and $g \in C(\partial \Omega)$ there exists an integer $n$ such that $n U_{\max } \geq g$. Moreover, $n U_{\max }$ is also a supersolution of the equation (91). Indeed,

$$
\begin{aligned}
0 & \geq n\left(\Delta U_{\max }-h U_{\max }-k \psi\left(U_{\max }\right)-f\right) \\
& =\Delta\left(n U_{\max }\right)-h\left(n U_{\max }\right)-k\left(n \psi\left(U_{\max }\right)\right)-f-(n-1) f \\
& \geq \Delta\left(n U_{\max }\right)-h\left(n U_{\max }\right)-k \psi\left(n U_{\max }\right)-f
\end{aligned}
$$

in the sense of distributions. Here we used convexity of $\psi$, which implies $\psi(n u) \geq$ $n \psi(u)$ for all $u \geq 0$, and the fact that $f \leq 0$.

Having this, we look for the solution to equation (91) satisfying the Dirichlet boundary data $g$ on $\partial \Omega$ in the closed convex set

$$
\mathcal{K}=\left\{u \in C(\bar{\Omega}): 0 \leq u \leq U_{\max }\right\} .
$$

Let $h_{1}=\max \{h, 0\}$ be the positive and $h_{2}=\max \{-h, 0\}$ be the negative part of the function $h$. Let us extend the function $\psi$ onto the whole $\mathbb{R}$ by putting $\psi(x)=-\psi(-x)$ for $x<0$. Then Theorem 5.2 implies that the map $T_{1}: \mathcal{K} \rightarrow C(\bar{\Omega})$ defined by $v=T_{1}(u)$, where $v$ is a solution to the PDE

$$
\Delta v-h_{1} v-k \psi(v)=f-h_{2} u \quad \text { in } \Omega, \quad v \in C(\bar{\Omega}),\left.\quad v\right|_{\partial \Omega}=g
$$

is well defined. Finally, take

$$
T: \mathcal{K} \rightarrow \mathcal{K}, \quad \text { where } \quad T(u)=\max \left\{0, \min \left\{U_{\max }, T_{1}(u)\right\}\right\} .
$$

We claim that the map $T$ is also continuous and compact. It suffices to prove that the map $T_{1}$ is continuous and compact. Let us first deal with the continuity. Take $u_{1}, u_{2} \in \mathcal{K}$ and let $v_{i}=T_{1}\left(u_{i}\right), i=1,2$. Consider $w=u_{1}-u_{2}$. Then $w$ is a solution to the linear PDE

$$
(\Delta-V) w=-h_{2}\left(u_{1}-u_{2}\right) \quad \text { in } \Omega, \quad w \in C(\bar{\Omega}),\left.\quad w\right|_{\partial \Omega}=0,
$$

where

$$
V(x)=h_{1}(x)+k(x)\left(\frac{\psi\left(v_{1}(x)\right)-\psi\left(v_{2}(x)\right)}{v_{1}(x)-v_{2}(x)}\right) \quad \text { for all } x \text { such that } v_{1}(x) \neq v_{2}(x) .
$$


We take $V(x)=h_{1}(x)$ for $v_{1}(x)=v_{2}(x)$. As before for the function $V_{12}$ it follows that $V \geq 0$ and $V \in L^{n / 2+\varepsilon}(\Omega)$. Hence, by the estimate (82), equation (96) gives us for $w$ :

$$
\|w\|_{C(\bar{\Omega})} \leq C\left\|h_{2}\left(u_{1}-u_{2}\right)\right\|_{L^{n / 2+\varepsilon}(\Omega)} \leq C\|h\|_{L^{n / 2+\varepsilon}(\Omega)}\left\|u_{1}-u_{2}\right\|_{C(\bar{\Omega})} .
$$

From this continuity follows. The proof of compactness of $T_{1}$ is very similar to the proof we gave for $T$ in the previous theorem. Therefore we just sketch the important details. Let $\left(u_{n}\right)_{n \in N}$ be a sequence from $\mathcal{K}$ and $v_{n}=T_{1}\left(u_{n}\right)$. It follows from equation (94) that all $v_{n}$ are uniformly bounded in the $C(\bar{\Omega})$ norm (by (82)). Hence

$$
\Delta v_{n}=f_{n}=f+h_{1} v_{n}-h_{2} u_{n}+k \psi\left(v_{n}\right) \quad \text { in } \Omega, \quad v_{n} \in C(\bar{\Omega}),\left.\quad v_{n}\right|_{\partial \Omega}=g .
$$

It follows that the norm of $f_{n}$ can be bounded independently of $n$. From this the compactness follows as the proof copies the argument given below (87).

Hence $T_{1}$ and therefore $T$ are continuous and compact. It follows that the map $T$ has a fixed point as the set $\mathcal{K}$ is closed and convex. Let $u \in \mathcal{K}$ be such a fixed point and let $w=T_{1}(u)$. We want to show that $u=w$; hence $u$ is a fixed point of $T_{1}$, i.e., a solution to (191) such that $\left.u\right|_{\partial \Omega}=g$. We leave the details to the reader. The main point of the proof uses the fact that $0 \leq u \leq U_{\max }$ and that 0 is a subsolution and $U_{\max }$ a supersolution.

The uniqueness follows from Lemma 4.1 under the additional assumptions on $f$ and $k$. Let $k>0$ in $\Omega$. Then, if $u_{1}, u_{2}$ are two solutions to our problem, the interior regularity results imply that $u_{1}, u_{2} \in L_{1, l o c}^{2}(\Omega)$. Hence $u_{1}, u_{2}$ satisfy all assumptions of Lemma 4.1 as well as (24). Let $\Omega_{0}=\left\{x \in \Omega: u_{1}(x)>u_{2}(x)\right\}$. Then if we apply Lemma 4.1 in domain $\Omega_{0}$, we have that $\left.u_{1}\right|_{\partial \Omega_{0}}=\left.u_{2}\right|_{\partial \Omega_{0}}$ and $u_{1}$ is a subsolution and $u_{2}$ is a supersolution. Hence $u_{1} \leq u_{2}$ in $\Omega_{0}$ from which it follows that $\Omega_{0}=\emptyset$. Therefore $u_{1} \leq u_{2}$ in $\Omega$. If we switch $u_{1}$ and $u_{2}$ we get $u_{2} \leq u_{1}$; hence $u_{1}=u_{2}$.

If instead we assume $k>0$ on a set of positive measure and $f, g, h \in C_{l o c}^{\alpha}(\Omega)$ for some $\alpha>0$, then interior regularity of the PDE implies that $u_{1}, u_{2} \in C^{2}(\Omega)$. We claim that unless $f, g$ are vanishing, then $u_{1}, u_{2}>0$ in $\Omega$ and hence uniqueness follows as above. This is clear, as in such a case $u \equiv 0$ is a subsolution but not a solution; hence by the second part of Lemma 4.1 we have that $u_{1}, u_{2}>0$ in $\Omega$.

Remark 5.1. If both $f$ and $g$ vanish, it is not difficult to construct an example of nonuniqueness.

Remark 5.2. Let $\psi(u) / u \rightarrow \infty$ as $u \rightarrow \infty$. Then the previous theorem implies the existence of a solution for arbitrary $g \in C(\partial \Omega), g \geq 0$, provided $h, k, f \in L^{\infty}(\Omega)$ and $k \geq c>0$. Indeed, find $M>0$ large enough such that $0 \leq g \leq M$ and $c \psi(M) \geq|h| M+|f|$. Then $U_{\max }=M$ is the desired supersolution; hence the existence and uniqueness follow.

Finally, we can establish the existence of large solutions for regular boundaries.

Theorem 5.4. Let $\Omega \subset M$ be a domain with nonempty boundary such that all boundary points are regular. Suppose that $\psi$ satisfies the standard assumptions. Then given any $h, k, f \in L^{\infty}(\Omega)$ such that $f \geq 0, k \geq c>0$ there exists a large 
nonnegative solution of the equation

$$
\Delta u+h u-k \psi(u)=-f, \quad \text { in } \Omega .
$$

Moreover, if $h, k, f \in C_{l o c}^{\alpha}(\Omega)$ the solution $u$ does not vanish.

If, in addition, $k$ is continuous, then the large solution is also unique.

Proof. By Theorem 5.3 there exists a unique solution $u_{n}$ to equation (4) such that $u_{n} \in C(\bar{\Omega})$ and $\left.u_{n}\right|_{\partial \Omega}=n$. Lemma 4.1 implies that $u_{1} \leq u_{2} \leq u_{3} \leq \ldots$ On the other hand, Corollary 4.4 implies that $u_{n} \leq B$; hence for every $x \in \Omega$ the limit $\lim _{n \rightarrow \infty} u_{n}(x)$ exists and is finite. Denote this limit by $u(x)$. It follows that the sequence $\left(u_{n}\right)_{n \in \mathbb{N}}$ converges locally uniformly and $u \in C_{l o c}(\Omega)$ solves (4). Finally, the continuity of $u_{n}$ in $\bar{\Omega}$ and the boundary condition of $u_{n}$ implies that given any $N>0$ there exists $\delta>0$ such that for all $x \in \Omega, \delta(x)=\operatorname{dist}(x, \partial \Omega)<\delta$, we have $u(x)>N$. Hence $u$ is the desired large solution.

If $f, g, h$ are more regular, Lemma 4.1 implies that $u_{n}>0$; hence $u>0$ in $\Omega$.

Now we do the uniqueness. Find a sequence of slightly smaller regular domains $\Omega_{\varepsilon} \Subset \Omega$ that approximate $\Omega$ from inside and go to $\Omega$ as $\varepsilon \rightarrow 0$. One can just take $\Omega_{\varepsilon}=\{x \in \partial \Omega: \operatorname{dist}(x, \partial \Omega)>\varepsilon\}$. Then we consider equation (4) on domain $\Omega_{\varepsilon}$ with boundary data $\left.u_{n}^{\varepsilon}\right|_{\partial \Omega}=n$. Now, at any point $y \in \Omega$, $\sup _{n}\left|u_{n}^{\varepsilon}(y)-u_{n}(y)\right| \rightarrow 0$ as $\varepsilon \rightarrow 0$. Two ingredients go into this estimate. The first one is that the variation in $k$ is small near the boundary; the second one is that boundaries $\Omega_{\varepsilon}$ and $\Omega$ are uniform in the sense that whatever estimate we can get for $\Omega$ will also hold on $\Omega_{\varepsilon}$ with a slightly larger constant. Now, let $v$ be any large solution in $\Omega$. Then at any $y \in \partial \Omega$ for fixed $\varepsilon$ and large $n$, we have by the comparison lemma,

$$
u_{n}(y) \leq v(y) \leq u_{n}^{\varepsilon}(y) .
$$

Hence taking the limit in $\varepsilon$ yields that both the left and right hand side converge to the same function, namely the large solution we found above. It follows that $v$ must be equal to this large solution.

\section{Localization PRINCIPLE (EXISTENCE)}

Let us recall that $u$ is called a large solution provided it satisfies (5). In this section we present proofs of the main results listed in section 3 on existence. In particular, we establish that the existence of a large solution is a local property; that is, the question whether a large solution exists for any domain $\Omega \subset M$ can be reduced to the same question for $U \cap \Omega$. Here $U$ is a neighborhood of a point $x \in \partial \Omega$. This is analogous to results for the linear operator $\Delta-V$ with continuous boundary data and is crucial in proving Theorem 2.7. We start by proving Theorem 3.1.

Proof of Theorem 3.1. One implication is clear: if $\Omega$ is $\psi$-regular, so are any of its boundary points. We only have to deal with one issue, namely, that in Definition 2.9, if $\psi$ satisfies the Harnack assumptions, then the existence of a large solution for the equation $\Delta u-c \psi(u)=0$ for one $c>0$ implies the existence for all $c>0$. For simplicity, let us assume that we have a large solution for $\Delta u-\psi(u)=0$ in $\Omega$. We want to look at $\Delta v-c \psi(v)=0$. In $c<1$ we are done as in such a case $u$ is going to be a subsolution for $\Delta v-c \psi(v)=0$. In such a case consider $\Theta_{1} \subset \Theta_{2} \subset \Theta_{3} \subset \ldots$, an increasing sequence of domains with smooth boundary 
such that $\overline{\Theta_{n}} \subset \Theta_{n+1} \subset \Omega$ and $\bigcup_{n \in \mathbb{N}} \Theta_{n}=\Omega$. Theorem 5.4 gives us that for each $n$ there is a large nonnegative solution $v_{n}$ of $\Delta v-c \psi(v)=0$ in $\Theta_{n}$. We note that it is possible that $\Theta_{n}$ are multiply connected domains; in such a case, Theorem 5.4 applies to each connected component of $\Theta_{n}$ separately. The comparison lemma implies that $\left(v_{n}\right)_{n \in \mathbb{N}}$ is monotone decreasing and $v_{n} \geq u$. It follows that $v=\lim _{n \rightarrow \infty} v_{n}$ is well defined on $\Omega$ and $v \geq u$ solves our equation. It also follows that $v$ must be a large solution.

If $c>1$ we need to use a different subsolution in the construction above. Consider $u / \theta$ instead, where $\theta>1$ is as in (8). It follows that for some $q>1$ and any $u>0$ : $\psi(u) \geq q \theta \psi(u / \theta)$. Hence

$$
0=\Delta(u / \theta)-\psi(u) / \theta \leq \Delta(u / \theta)-q \psi(u / \theta)
$$

Hence $u / \theta$ is a subsolution of $\Delta u-c \psi(u)=0$ for all $c \leq q$. By a similar argument, $u / \theta^{n}$ is a subsolution for $\Delta u-c \psi(u)=0$ for all $c \leq q^{n}$. The rest goes as above.

The converse follows from the following lemma.

Lemma 6.1. Let $x$ be a $\psi$-regular point of the domain $\Omega$. Then there exists a neighborhood $U$ of $x$ such that for any $c>0$ the equation $\Delta u-c \psi(u)=0$ has a positive solution in $\Omega \cap U$ with the following properties:

(a) $u(x) \rightarrow \infty$ locally uniformly as $x \rightarrow \Gamma_{1}=\partial \Omega \cap U$.

(b) $u$ is continuous in $\Omega \cup \Gamma_{2}$ and $u=0$ on $\Gamma_{2}=\Omega \cap \partial U$.

Assume for a second that the lemma holds. Pick any $c>0$. Consider again the equation $\Delta u-c \psi(u)=0$ in $\Omega$. We want to construct a large solution in $\Omega$. Consider the same sequence $\overline{\Theta_{n}} \subset \Theta_{n+1} \subset \Omega$ and $\bigcup_{n \in \mathbb{N}} \Theta_{n}=\Omega$ of smooth subdomains as above. Theorem 5.4 again gives us that for each $n$ there is a large nonnegative solution $u_{n}$ of $\Delta u_{n}-c \psi\left(u_{n}\right)=0$ in $\Theta_{n}$. As before, by the comparison theorem, $u_{1} \geq u_{2} \geq u_{3} \geq \ldots$; hence the limit $u=\lim _{n \rightarrow \infty} u_{n}$ exists and solves our equation in $\Omega$. It remains to prove that $u$ is a large solution. Every point of $\partial \Omega$ is $\psi$-regular; hence (by the lemma) every point has a neighborhood $U$ such that in $U \cap \Omega$, (a) and (b) hold. As $\partial \Omega$ is compact we can find a finite number of open sets $U_{1}, U_{2}, \ldots, U_{m}$ covering $\partial \Omega$ such that $v_{i}, i=1,2, \ldots, m$ is a solution satisfying (a) and (b) in $\Omega \cap U_{i}$. Using the comparison lemma in $\Omega \cap U_{i}$ we see that $u_{n} \geq v_{i}$ for all $n$ and $i$. Hence by taking the limit we see that $u \geq v_{i}$ in $\Omega \cap U_{i}$. As sets $U_{i}$ cover the boundary we have by (a) that $u(x) \rightarrow \infty$ as $x \rightarrow \partial \Omega$, so $u$ is a large solution.

The lemma above as well as Theorem 3.2, part (a) will be proven if we establish the following proposition.

Proposition 6.2. Let $\Omega \subset M$ be an arbitrary domain with nonempty boundary. Suppose that $\psi$ satisfies the Harnack assumptions and $c>0$ is given. Then the following statements are equivalent:

(i) Domain $\Omega$ is $\psi$-regular.

(ii) For every $h, k, f \in L^{\infty}(\Omega)$ such that $f \geq 0, k \geq c>0$ there exists a large nonnegative solution of equation (4) in $\Omega$.

(iii) Let $h, k, f \in L^{\infty}(\Omega)$ be given, such that $f \geq 0, k \geq c>0$. There exists $\delta>0$ such that for every $x \in \partial \Omega$ and a neighborhood $U \subset B(x, \delta)$ with $C^{1}$ boundary equation (4) has a large nonnegative solution in $\Omega \cap U$. 
(iv) Let $h, k, f \in L^{\infty}(\Omega)$ be the same as in (ii). There exists $\delta>0$ such that for every $x \in \partial \Omega$ and a neighborhood $U \subset B(x, \delta)$ with $C^{1}$ boundary we can find a nonnegative solution $u$ of (4) in $\Omega \cap U$ satisfying:e

(a) $u(x) \rightarrow \infty$ locally uniformly as $x \rightarrow \Gamma_{1}=\partial \Omega \cap U$.

(b) $u$ is continuous in $\Omega \cup \Gamma_{2}$ and $u=0$ on $\Gamma_{2}=\Omega \cap \partial U$.

If $\psi$ only satisfies the standard assumptions, we have (i) $\Longrightarrow$ (ii) $\Longrightarrow$ (iii) $\Longrightarrow$ (iv).

Proof. (i) $\Longrightarrow$ (ii). Let $c=2 \sup _{\Omega} k$ and $b=\sup \left\{-h(x) t-\frac{1}{2} c \psi(t): t>0, x \in \Omega\right\}$. Then every positive solution of (4) satisfies

$$
\Delta u-c \psi(u) \leq b .
$$

Take $U_{\max }$ to be a large solution of $\Delta U_{\max }-2 c \psi\left(U_{\max }\right)=0$ in $\Omega$. Consider $M(\delta)=$ $\inf \left\{U_{\max }(x): x \in \Omega\right.$ and $\left.\delta(x)<\delta\right\}$. Then, since $U_{\max }$ is the large solution there exists $\delta>0$ such that $M=M(\delta)$ satisfies $c \psi(M) \geq b$. Then in a neighborhood $U=\{x \in M: \operatorname{dist}(x, \partial \Omega)<\delta\}$ we have

$$
\Delta U_{\max }-c \psi\left(U_{\max }\right) \geq b \text { in } \Omega \cap U .
$$

Let $\Theta_{1} \subset \Theta_{2} \subset \Theta_{3} \subset \ldots$ by an increasing sequence of domains with smooth boundary such that $\overline{\Theta_{n}} \subset \Theta_{n+1} \subset \Omega$ and $\bigcup_{n \in \mathbb{N}} \Theta_{n}=\Omega$. Theorem 5.4 gives us that for each $n$ there is a large nonnegative solution $u_{n}$ of (4) in $\Theta_{n}$. Again, the comparison lemma implies that $\left(u_{n}\right)_{n \in \mathbb{N}}$ is monotone decreasing. It follows that $u=\lim _{n \rightarrow \infty} u_{n}$ is well defined on $\Omega$ and $u$ solves (4). It remains to show that $u$ is a large solution. Consider any smooth open set $V \subset U$ containing a point $x \in \partial \Omega$. Let $v$ be a large solution of $\Delta v-c \psi(v)=0$ in $V$. Consider $u_{n}+v$ in $\Theta_{n} \cap V$. It follows from (101) and (102) that

$$
\Delta\left(u_{n}+v\right)-c \psi\left(u_{n}+v\right) \leq \Delta u_{n}-c \psi\left(u_{n}\right)+\Delta v-c \psi(v) \leq \Delta U_{\max }-c \psi\left(U_{\max }\right) ;
$$

hence by the comparison lemma, if $u_{n}+v \geq U_{\max }$ on $\partial\left(\Theta_{n} \cap V\right)$, then the same holds inside. However on $\partial\left(\Theta_{n} \cap V\right), u_{n}+v$ is infinite and $U_{\max }$ is finite, so the inequality holds.

Limiting $u_{n}+v \geq U_{\max }$ we get $u+v \geq U_{\max }$ in $\Omega \cap V$ or $u \geq U_{\max }-v$. However, as $v$ is finite near $x$, we get that for all points on $\partial \Omega$ near $x, u$ goes to infinity when approaching the boundary. As this is true near any boundary point, $u$ must be a large solution of (4).

(ii) $\Longrightarrow$ (iii). Let $U$ be any set with $C^{1}$ boundary containing a point from $\partial \Omega$. Denote by $U_{\max }$ the large solution of equation (4) in $\Omega$ from (ii). Denote by $v$ the large solution of the same equation in $U$ (Theorem 5.4). Consider the sets with smooth boundary $\Theta_{1} \subset \Theta_{2} \subset \Theta_{3} \subset \ldots$ now exhausting $\Omega \cap U$. As before we denote by $u_{n}$ the solution of equation (4) in $\Theta_{n}$. Let $u=\lim _{n \rightarrow \infty} u_{n}$. By the same argument as given above, $u$ is well defined in $\Omega \cap U$ and solves the equation. It remain to establish that $u$ is a large solution. This has two parts. On $\Theta_{n}$ we use comparison of $u_{n}$ with $U_{\max }$. As $\Theta_{n} \Subset \Omega$ and both solve the same equation we see that $u_{n} \geq U_{\max }$ as this holds on $\partial \Theta$. From this, $u_{n} \geq U_{\max }$ on $\Omega \cap U$. The same argument as given above is also true when $U_{\max }$ is replaced by $v$. Hence $u \geq \max \left\{U_{\max }, v\right\}$ and so $u(x) \rightarrow \infty$ as $x \rightarrow \partial(\Omega \cap U)$.

(iii) $\Longrightarrow$ (iv). Notice that the claim does not say what happens on $\partial U \cap \partial \Omega$. Take $x$ and $U$ as in (iii). Let $\Theta_{1} \subset \Theta_{2} \subset \Theta_{3} \subset \ldots$ be an increasing sequence of domains with $C^{1}$ boundary such that $\bigcup_{n \in \mathbb{N}} \Theta_{n}=\Omega \cap U$. This time we require that

$$
\Omega \backslash K_{n} \subset \Theta_{n}, \quad \text { where } K_{n}=\left\{x \in M: \operatorname{dist}\left(x, \Gamma_{1}\right)<1 / n\right\} .
$$


Let $\Gamma_{1, n}=\partial \Theta_{n} \cap K_{n}, \Gamma_{2, n}=\partial \Theta_{n} \cap\left(M \backslash \overline{K_{n}}\right)$. It follows that $\Gamma_{2, n} \subset \Gamma_{2, n+1} \subset \Gamma_{2}$. In addition, matters can be arranged such that all the $\Gamma_{1, n}$ are disjoint.

For every $n$, we consider a sequence of continuous functions $\left(\varphi_{n, k}\right)_{k \in \mathbb{N}}$ on $\partial \Theta_{n}$. We require

$$
\begin{aligned}
& \varphi_{n, k}=k \quad \text { on } \Gamma_{1, n}, \quad \varphi_{n, k}=0 \quad \text { on }\left\{x \in \Gamma_{2, n}: \operatorname{dist}\left(x, \Gamma_{1, n}\right)>1 / n\right\} \\
& 0 \leq \varphi_{n, k} \leq k \quad \text { on } \partial \Theta_{n}, \\
& \varphi_{n, k} \leq \varphi_{n, k+1} \quad \text { on } \partial \Theta_{n}, \quad \varphi_{n, k} \geq \varphi_{n+1, k} \quad \text { on } \Gamma_{2, n} .
\end{aligned}
$$

Theorem 5.3 implies that for each $n$ and $k$ there is a nonnegative and continuous solution $u_{n, k}$ of (44) on $\overline{\Theta_{n}}$ equal to $\varphi_{n, k}$ at the boundary. As in the proof of Theorem 5.4 it follows that $u_{n}=\lim _{k \rightarrow \infty} u_{n, k}$ is well defined, since $\left(u_{n, k}\right)_{k \in \mathbb{N}}$ is a monotone increasing and bounded sequence (by the barrier $B$ for domain $\Omega$ ). It follows that $u_{n} \in C_{l o c}\left(\Theta_{n}\right)$ solves (4) in $\Theta_{n}$. Also $u_{n} \rightarrow \infty$ as $x \rightarrow \Gamma_{1, n}$. It requires a bit more sophisticated argument to show that $u_{n}$ is continuous on $\Gamma_{2, n}^{\prime}=\left\{x \in \Gamma_{2, n}: \operatorname{dist}\left(x, \Gamma_{1, n}\right)>1 / n\right\}$ and $\left.u\right|_{\Gamma_{2, n}^{\prime}}=0$. This requires improving the barrier function $B$ such that it is vanishing on $\Gamma_{2, n}^{\prime}$. This can be arranged by taking $B^{\prime}$ to be a nonnegative solution to (4) on a smaller domain $\Theta_{n}^{\prime} \subset \Theta_{n}$. Here we take $\Theta_{n}^{\prime}$ such that its boundary is $C^{1}$, and $\partial \Theta_{n}^{\prime}=\Gamma_{2, n}^{\prime} \cup X_{n}$, where $X_{n} \Subset \Theta_{n}$. We also require $\left.B^{\prime}\right|_{X_{n}}=\left.B\right|_{X_{n}}$ and $\left.B^{\prime}\right|_{\Gamma_{2, n}^{\prime} \backslash\left\{x \in M: \operatorname{dist}\left(x, X_{n}\right)<\varepsilon\right\}}=0$ for any $\varepsilon>0$. On the rest of the boundary we prescribe the boundary data such that the overall function is continuous. Theorem 5.3 implies existence of such a $B^{\prime}$ and the comparison lemma gives $u_{n, k} \leq B^{\prime}$ in $\Theta_{n}^{\prime}$ as $u_{n, k} \leq B^{\prime}$ on $\partial \Theta_{n}^{\prime}$. From this we get continuity of $u_{n}$ on $\Gamma_{2, n}^{\prime} \backslash\left\{x \in M: \operatorname{dist}\left(x, X_{n}\right)<\varepsilon\right\}$. As $\varepsilon>0$ can be taken arbitrarily small, the claim holds on the whole $\Gamma_{2, n}^{\prime}$.

Next, we take the limit $n \rightarrow \infty$. As $u_{n, k} \geq u_{n+1, k}$ the sequence $\left(u_{n}\right)_{n \in \mathbb{N}}$ is monotone decreasing. Consequently, $u=\lim _{n \rightarrow \infty} u_{n}$ is well defined in $U \cap \Omega$ and $u$ solves (44) there. Given the fact that $u_{n}$ is continuous and vanishes on $\Gamma_{2, n}^{\prime}$ we see that $u$ is continuous and vanishes on $\bigcup_{n \in \mathbb{N}} \Gamma_{2, n}^{\prime}=\Gamma_{2}$. This gives us (b).

What happens on $\Gamma_{1}$ ? Let $V$ be the large solution of (44) in $U$ and let $W$ be the large solution of the same equation in $\Omega \cap U$ (which exists thanks to our assumption that (ii) holds). It follows that $u_{n}+V$ is a supersolution of our equation in $\Theta_{n}$ that blows up on the whole $\partial \Theta_{n}$; hence $u_{n}+V \geq W$. From this, $u+V \geq W$. As $V$ stays bounded on the set $\left\{x \in \Gamma_{1}: \operatorname{dist}(x, \partial U)>\varepsilon\right\}$ for any $\varepsilon>0$ we conclude that $u \geq W-V \rightarrow \infty$ uniformly on such a set. This implies (a).

(iv) $\Longrightarrow$ (i). Now we assume the stronger condition on $\psi$ (Harnack). This allows us to deduce that $\Omega$ is a $\psi$-regular domain if the equation $\Delta u-\frac{c}{2} \psi(u)=0$ has a large solution in $\Omega \cap U_{x}$, for all $x \in \partial \Omega$ and some neighborhood $U_{x}$ of $x$. Consider $\delta>0$ as in (iv). Pick $x \in \partial \Omega$ and take $U=B(x, \delta)$ and find the solution $u$ in $\Omega \cap U$ satisfying (a) and (b) and solving (4) for the given functions $f, g, h$. From (a) it follows that there is $0<\delta^{\prime}<\delta$ such that in $B\left(x, \delta^{\prime}\right) \cap \Omega, u$ is very large and therefore $\frac{c}{2} \psi(u) \geq|h u|+|f|$. It follows that in $B\left(x, \delta^{\prime}\right) \cap \Omega$, $u$ satisfies $\Delta u-\frac{c}{2} \psi(u) \geq 0$, or $u$ is a subsolution of the equation $\Delta v-\frac{c}{2} \psi(v)=0$.

The rest is standard. We exhaust set $B\left(x, \delta^{\prime}\right) \cap \Omega$ by an increasing sequence of smooth domains $\Theta_{1} \subset \Theta_{2} \subset \Theta_{3} \subset \ldots$ and on each we find a large solution $u_{n}$ of the equation $\Delta v-\frac{c}{2} \psi(v)=0$. It follows that $u_{n} \geq u$ and $u_{n} \geq w$ on $\Theta_{n}$. Here $w$ is a large solution in $B\left(x, \delta^{\prime}\right)$. Limiting $n \rightarrow \infty$ we get that $U=\lim _{n \rightarrow \infty} \geq \max \{u, w\}$; hence $U$ solves $\Delta u-\frac{c}{2} \psi(u)=0$ in $B\left(x, \delta^{\prime}\right) \cap \Omega$ and $U$ is a large solution. 
Proof of the rest of Theorem 3.2. We first address the issue of the largest maximal solution of equation (44). Let $u$ be any nonnegative solution of equation (4). Consider as before an increasing sequence of domains with smooth boundary $\Theta_{1} \subset \Theta_{2} \subset \cdots \subset \Omega$ whose union is $\Omega$. We also require $\overline{\Theta_{n}} \subset \Theta_{n+1}$. Then on each $\Theta_{n}$ we find unique large nonnegative solutions $u_{n}$ of equation (44) in $\Theta_{n}$. As before, Lemma 4.1 gives us that $u_{n} \geq u_{n+1}$ and $u_{n} \geq u$ as both $u_{n+1}$ and $u$ are bounded on $\Theta_{n}$ and $u_{n}$ blows up at the boundary of $\Theta_{n}$. It follows that $U_{\max }=\lim _{n \rightarrow \infty} u_{n}$ is well defined and solves (4) in $\Omega$. Also $U_{\max } \geq u$. As $u$ is arbitrary, $U_{\max }$ must be a large solution, since $\Omega$ has at least one large solution.

Regarding $U_{\min }$, we claim that the solution we constructed in the proof of Theorem 5.4 is minimal. Indeed, if $u_{n}$ is the sequence of functions on $\Omega$ such that $\left.u_{n}\right|_{\partial \Omega}=n$, then $u_{n} \leq u$ for any large solution $u$ on $\Omega$. Hence $U_{\min }=\lim _{n \rightarrow \infty} u_{n} \leq$ $u$.

Now we make the connection between $\psi$-regularity and the concept of local barriers, which are defined in Theorem 2.7.

Proof of Theorem 3.3. The implication $\Longrightarrow$ is trivial, since if $x$ is $\psi$-regular, then assuming $U$ has a "nice" boundary, $\Omega \cap U$ is $\psi$-regular and hence the large solution of $\Delta u-\psi(u)=0$ in $U \cap \Omega$ is a $\psi$-barrier at $x$.

Conversely, we first make the standard construction by finding a sequence of smooth domains exhausting $\Omega$ by a sequence of smooth domains on which we can find a large solution $\left(u_{n}\right)$ of $\Delta u-\psi(u)=0$. Let $w$ be a $\psi$-barrier at $x$. Without loss of generality (by making $U$ smaller if necessary) we can assume that $U$ has $C^{1}$ boundary and $w \in L_{l o c}^{\infty}(\partial U \cap \Omega)$. Let $v$ be a large solution of the same equation on $U$. It follows from the comparison lemma that $u_{n}$ is a decreasing sequence of functions and therefore $u=\lim _{n \rightarrow \infty} u_{n}$ exists and solves our equation. Also by the comparison lemma, $w \leq u_{n}+v$, as $w$ is a subsolution, $u_{n}+v$ a supersolution and the inequality holds at the boundary. Limiting yields $w \leq u+v$ from which $u(y) \rightarrow \infty$ as $y \rightarrow x$ since $v$ is bounded near $x$. This coupled with the fact that $\partial \Omega$ is a compact set implies that $u(y) \rightarrow \infty$ uniformly as $y \rightarrow \partial \Omega$, so $\Omega$ is a $\psi$-regular domain.

\section{LARGE SOLUTIONS AND NONUNIQUENESS ON A LARGE ClASS OF FRACTAL DOMAINS}

In this section we present a method to construct large solutions for a broad class of domains $\Omega$ and functions $\psi$. The method is a generalization of the construction from [45, 6], 14] and [16. These papers established that on domains $M \backslash \Gamma$, where $\Gamma$ is a smooth submanifold of dimension $d \leq n-2$, there are examples of nonuniqueness of large solutions.

One important aspect of this generalization is that it applies to domains with fractal boundaries and shows nonuniqueness for such domains.

Lemma 7.1. Let $\Gamma \subset M$ be a closed set of Hausdorff dimension $d \leq n-2$ such that $H^{d}(\Gamma)>0$ and

$$
H^{d}(\Gamma \cap B(x, r)) \leq c r^{d},
$$

for all $x \in \Gamma$ and $0<r<\operatorname{diam}(M)$ and some $c$ independent of $x$ and $r$. Here $H^{d}$ denotes the d-dimensional Hausdorff measure on $M$. 
Then there exists a positive function $u$ on $M \backslash \Gamma$ solving $(\Delta-1) u=0$ in $M \backslash \Gamma$ and $u$ satisfies $\lim _{y \rightarrow x} u(y)=\infty$ for all $x \in \Gamma$ such that $\lim _{\sup } \rightarrow 0+r^{-d} H^{d}(\Gamma \cap$ $B(x, r))>0$.

Moreover, there exists $c>0$ such that for all $x \in M \backslash \Gamma$ with $\operatorname{dist}(x, \Gamma) \leq 1 / 2$ :

$$
u(x) \leq \begin{cases}-c \ln \operatorname{dist}(x, \Gamma), & \text { if } d=n-2, \\ c(\operatorname{dist}(x, \Gamma))^{-(n-2-d),}, & \text { if } d<n-2 .\end{cases}
$$

Proof. The mapping properties of the operator $(1-\Delta)^{-1}$ have been studied extensively in [35-38 and also in 9. In particular, this operator is invertible under very mild assumptions on the metric tensor on $M$ and the fundamental solution $E(x, y)$; i.e., a function solving $\left(1-\Delta_{x}\right) E(x, y)=\delta_{y}(x)$ in $M$ has the form

$$
E(x, y)= \begin{cases}-c_{2} \ln (\operatorname{dist}(x, y))+\text { less singular term, } & \text { if } n=2, \\ c_{n}(\operatorname{dist}(x, \Gamma))^{-(n-2)}+\text { less singular term, } & \text { if } n>2,\end{cases}
$$

near the diagonal $x=y$ for some positive $c_{n}>0$. In particular, $E(x, y) \in$ $L_{l o c}^{\infty}(M \times M \backslash\{(x, y): x=y\})$. Also, $E(x, y)>0$ as $1-\Delta$ is a positive operator. Consider:

$$
u(x)=\int_{\Gamma} E(x, y) d H^{d}(y), \quad \text { for } x \in M \backslash \Gamma .
$$

Since $\Gamma$ is closed, if $x \in M \backslash \Gamma$, it follows that $\operatorname{dist}(x, \Gamma)>0$. Hence for such an $x$, (108) is well defined as we integrate a bounded function over a set of finite and positive $d$-dimensional Hausdorff measure. We want to see that (106) holds. If $n=2$, i.e., $d=0$ there is nothing to prove. In all other cases, consider $x \in M \backslash \Gamma$ such that $0<\delta(x)=\operatorname{dist}(x, \Gamma)<1 / 2$. As (107) holds, the asymptotic of $u$ near $\Gamma$ is determined only by the leading order term of $E(x, y)$. Let $y \in \Gamma$ be a point such that $\operatorname{dist}(x, y)=\delta(x)$. Consider a sequence of balls

$$
B_{0}=\emptyset, \quad B_{k}=B\left(y, 2^{k} \delta(x)\right), \quad k=1,2, \ldots
$$

It follows that if $z \in \Gamma \cap\left(B_{k} \backslash B_{k-1}\right)$, then $2^{k-1} \delta(x) \leq \operatorname{dist}(x, z) \leq 2^{k+1} \delta(x)$. Hence

$$
\int_{\Gamma \cap\left(B_{k} \backslash B_{k-1}\right)} E(x, y) d H^{d}(y) \leq C \frac{\delta(x)^{d} 2^{(k+1) d}}{\delta(x)^{n-2} 2^{(n-2)(k-1)}}=C \delta^{-(n-2-d)} 2^{k(d-(n-2))} .
$$

Here we use (105) to estimate $H^{d}\left(\Gamma \cap\left(B_{k} \backslash B_{k-1}\right)\right)$ from above by $c_{2} \delta(x)^{d} 2^{(k+1) d}$.

If $d<(n-2)$, then if we sum the right hand side over $k \in \mathbb{N}$ we get a finite number, as the series is summable. From this, (106) follows. On the other hand, if $d=n-2$ we have to be more careful and sum only over $k$ such that $2^{k} \delta(x) \leq 1$. Clearly, the contribution of points $z \in \Gamma$ to the integral (108) for which $\operatorname{dist}(x, z)>$ $1 / 2$ is estimable by a constant independent of $x$. Hence,

$$
u(x) \leq C+\sum_{k=1}^{\left[-\log _{2} \delta(x)\right]} \int_{\Gamma \cap\left(B_{k} \backslash B_{k-1}\right)} E(x, y) d H^{d}(y) \leq-C \ln \delta(x) .
$$

This proves (106). It remains to prove the last part of our statement, that $u$ blows up at points $x \in \Gamma$ where $\lim \sup _{r \rightarrow 0+} r^{-d} H^{d}(\Gamma \cap B(x, r))>0$. Let $y \in M \backslash \Gamma$ such that $\operatorname{dist}(x, y)<r$. Then by (108), $u(y) \geq r^{n-2} H^{p}(\Gamma \cap B(x, r)) \geq r^{n-2-d} \rightarrow \infty$ if $d<n-2$ and $r \rightarrow \infty$. If $d=n-2$ the proof requires a bit more effort, but still comes through. 
Corollary 7.2. Let $\Gamma \subset M$ be a d-dimensional closed set such that it can be written as $\Gamma=\bigcup_{k=1}^{\infty} \Gamma_{k}$ where on $\Gamma_{k}$ we have

$$
H^{d}\left(\Gamma_{k} \cap B(x, r)\right) \leq c(k) r^{d}, \quad \text { for all } x \in \Gamma_{k} \text { and } r>0 .
$$

Also assume that for each $x \in \Gamma$ there is a $k$ such that $\lim _{\sup } \rightarrow 0+r^{-d} H^{d}\left(\Gamma_{k} \cap\right.$ $B(x, r))>0$. Then there is a large solution $u$ of the equation $(\Delta-1) u=0$ in $M \backslash \Gamma$ satisfying (106).

Proof. Let $\Sigma_{k}=\overline{\Gamma_{k}}$. Then $\Sigma_{k}$ satisfies all assumptions of Lemma 7.1. Letting $u_{k}$ be the function constructed in the lemma, it follows that $u_{k}$ satisfies (106) for some $c=c(k)>0$. Take $u=\sum_{k=1}^{\infty} u_{k} /\left(2^{k} c(k)\right)$. It follows that $u$ also satisfies (106) for $c=1$. If $x \in \Gamma$, then $u$ blows up at $x$ as our assumption implies that $u \geq u_{k} /\left(2^{k} c(k)\right)$ for all $k$ and $u_{k}$ blows up at $x$ for at least one $k$.

The following lemma is a simple corollary of the definition of the Hausdorff measure $H^{d}$.

Lemma 7.3. Let $\Gamma \subset M$ be an arbitrary set such that $H^{d}(\Gamma)<\infty$. Then there is $C>0$ such that

$$
H^{n}(\{x \in M: \operatorname{dist}(x, \Gamma)<r\}) \leq C r^{n-d}, \quad \text { for all } 0<r<1 .
$$

Theorem 7.4. Let $\Omega \subset M$ be a domain in $M$ such that $\Gamma=M \backslash \Omega$ satisfies all the assumptions of Corollary 7.2, Let $u$ be the function from Corollary 7.2 defined on $\Omega$. Assume that the function $\psi$ satisfies (6) and (7).

(a) If there exists $\varepsilon>0$ such that

$$
\lim _{r \rightarrow 0+} \sup _{x \in M} \int_{B(x, r)} E(x, y)\left[\psi(\varepsilon u(y))+\psi^{\prime}(\varepsilon u(y))\right] d \operatorname{Vol}(y)=0,
$$

then $\Omega$ is a $\psi$-regular domain. Here $E(x, y)$ is defined in (107). Moreover, there are infinitely many large positive solutions of the equation $(\Delta-1) v-\psi(v)=0$ in $\Omega$ of the form $v=c u+w_{c}$ for $0<c<\varepsilon$ and $w_{c} \in C(M)$.

(b) If there exists $\varepsilon>0$ and $r>0$ such that:

$$
\lim _{x \in \Omega, x \rightarrow \Gamma} \frac{1}{u(x)} \int_{\{y \in \Omega ; \delta(y)<r\}} E(x, y)\left[\psi(\varepsilon u(y))+\psi^{\prime}(\varepsilon u(y))\right] d \operatorname{Vol}(y)=0,
$$

then $\Omega$ is a $\psi$-regular domain. Moreover, there exist infinitely many large positive solutions of the equation $(\Delta-1) v-\psi(v)=0$ in $\Omega$ of the form $v=\left(c+w_{c}\right) u$ for $0<c<\varepsilon$ and some $w_{c} \in C(M)$ such that $\left.w_{c}\right|_{\Gamma}=0$.

Remark 7.1. The important aspect of this theorem is the nonuniqueness; that is, we construct a whole family of solutions for the parameter $c \in(0, \varepsilon)$.

Proof. Let us assume (a). Pick $0<c<\varepsilon$. Consider $x \in \Gamma$. Then for any bounded $w$ we see that $0<c u+w \leq \varepsilon u$ for $y$ close to $x$ as $u(y) \rightarrow \infty$ for $y \rightarrow x$. It follows that (5.19) holds for $\varepsilon u$ replaced by $c u+w$.

For $\delta>0$ consider $\mathcal{S}=\{w \in C(B(x, \delta)): w \leq 0\}$ and a map $T: \mathcal{S} \rightarrow \mathcal{S}$ defined as follows:

$$
T w(z)=-\int_{B(x, \delta)} E(z, y) \psi(c u(y)+w(y)) d \operatorname{Vol}(y), \quad \text { for } z \in B(x, \delta) .
$$

We see that (114) guarantees that $T w$ is a bounded function. It is also negative as $\psi(c u+w) \geq 0$ and $E(x, y) \geq 0$. Tw is continuous as for any $z, z^{\prime} \in B(x, \delta)$ close to 
each other the difference $E\left(z^{\prime}, y\right)-E(z, y)$ is small everywhere except at points $y$ close to $z$ or $z^{\prime}$. At those points we use (114) for small $r$. Let $\|w\|_{L^{\infty}} \leq 1$. Again by (114) we see that $\|T w\|_{L^{\infty}} \leq 1$, provided $\delta>0$ is chosen sufficiently small. Hence $T$ maps the set $\mathcal{C}=\mathcal{S} \cap\left\{w:\|w\|_{L^{\infty}} \leq 1\right\}$ into itself. Next we want to show that $T$ has a fixed point. We show that $T$ is contractive. We use the fact that

$$
\begin{aligned}
\left|\psi\left(c u+w_{1}\right)-\psi\left(c u+w_{2}\right)\right| & \leq \int_{0}^{1} \psi^{\prime}\left(c u+t w_{1}+(1-t) w_{2}\right)\left|w_{1}-w_{2}\right| \\
& \leq \psi^{\prime}(\varepsilon u)\left|w_{1}-w_{2}\right| .
\end{aligned}
$$

Hence $\left|T w_{1}(z)-T w_{2}(z)\right| \leq 1 / 2\left\|w_{1}-w_{2}\right\|$, provided $\delta>0$ is sufficiently small, i.e. the integral in (114) is less than $1 / 2$. So $T$ has a fixed point $T w=w$. Applying $\Delta-1$ to (116) we get that $(\Delta-1) w=\Delta(c u+w)=\psi(c u+w)$. So we have a family of solutions $c u+w>0$ on $B(x, \delta)$. Now we refer to Lemma 8.2, which shows that there is $u_{0}$, a solution of $(\Delta-1) u_{0}-\psi\left(u_{0}\right)=0$ vanishing on $\partial B(x, \delta) \cap \Omega$ such that $u_{0} \leq c u+w \leq u_{0}+U_{0}$ in $B(x, \delta) \cap \Omega$, where $U_{0}$ is a large solution in $B(x, \delta)$. We can extend $u_{0}$ continuously to the whole $\Omega$ by putting $u_{0}=0$ outside $B(x, \delta)$. This makes $u_{0}$ a subsolution of our equation in $\Omega$. Now, as we can do the same for all other points of $x \in \Gamma$ and $\Gamma$ is a compact set we eventually get subsolutions $u_{0}, u_{1}, u_{2}, \ldots, u_{k}$ such that for every $x \in \Gamma$ there is $i \in\{0,1,2, \ldots, k\}$ such that $u_{i}=c u+w_{i}$ near $x$. Finally consider $V=\max \left\{u_{0}, u_{1}, \ldots, u_{k}\right\}$. Then $V$ is also a subsolution and $V=c u+w$ for some bounded positive $w$ near $\Gamma$. Hence the set

$$
\begin{array}{ll}
\mathcal{U}=\{v: \Omega \rightarrow \mathbb{R}: \quad & v \text { is a subsolution of }(\Delta-1) u-\psi(u)=0 \\
& \text { and } \left.u \leq u_{i}+U_{i} \text { for } i=0,1, \ldots, k\right\}
\end{array}
$$

is nonempty as $V \in \mathcal{U}$. Finally take $U(x)=\sup _{v \in \mathcal{U}} v(x)$. By a standard argument, $U$ is a solution of the equation and $V \leq U \leq u_{i}+U_{i}$ on $B(x, \delta) \cap \Omega$. Hence $U=c u+w$ near $\Gamma$ for some bounded and positive $w$. So $U$ is a large solution in $\Omega$.

We can repeat the whole process for the equation $(\Delta-1) u-c \psi(u)=0$ for any $c>0$. So $(\Delta-1) u-c \psi(u)=0$ has a large solution for any $c>0$; i.e., $\Omega$ is a $\psi$-regular domain. This concludes our proof of case (a).

If (b) holds we consider instead the operator

$$
T w(x)=\frac{-1}{u(z)} \int_{\{y \in \Omega ; \delta(y)<r\}} E(x, y) \psi((c+w(y)) u(y)) d \operatorname{Vol}(y),
$$

defined on $\{y \in \Omega: \delta(y)<r\} \cap \Omega$. Assume $\|w\|_{L^{\infty}} \leq \varepsilon-c>0$, i.e., again $(c+w) u \leq \varepsilon u$, so we can use (115). It follows that $\lim _{x \rightarrow \Gamma} T w(x)=0$, so $\left.T w\right|_{\Gamma}=0$. If necessary, making $r>0$ smaller gives us that $\|T w\|_{L^{\infty}} \leq \varepsilon-c$ and $\| T w_{1}-$ $T w_{2} \|_{L^{\infty}} \leq 1 / 2$. So $T$ is contractive and has a fixed point. The rest goes as in case (a).

Proof of the claim in Example 3.1. It suffices to apply part (a) of Theorem 7.4 to $\Gamma_{i}$. This gives us a solution $u_{i}$ in $M \backslash \Gamma_{i}$ which can serve as a subsolution for the largest solution $U_{\max }$ in $\Omega$ (see Theorem 3.2); i.e., $U_{\max } \geq u_{i}$. Since for every $x \in \Gamma$ there is $i$ such that $u_{i}(y) \rightarrow \infty$ as $y \rightarrow x$, we get that $U_{\max }(y) \rightarrow \infty$ as $y \rightarrow \Gamma$; i.e., $U_{\max }$ is a large solution on $\Gamma$.

Assume therefore that $\Gamma$ satisfies (105) and let $u$ be as in Lemma 7.1. We want to establish (114). We note that $E(x, y) \in L^{n /(n-2)-\varepsilon}(M)$ in the variable $y$ uniformly in $x$ for any $\varepsilon>0$. Using this and Hölder's inequality we get that (114) certainly 
holds if

$$
\int_{\Omega}\left[\psi(\varepsilon u(y))+\psi^{\prime}(\varepsilon u(y))\right]^{\beta} d \operatorname{Vol}(y)<\infty
$$

for some $\beta>n / 2$. First consider the case when $\psi(x), \psi^{\prime}(x) \leq C e^{k x}$ for $x>0$. By (106) it suffices to show that

$$
\int_{\Omega} \frac{1}{\operatorname{dist}(x, \Gamma)^{\beta k \varepsilon}} d \operatorname{Vol}(y)<\infty
$$

We pick $\varepsilon$ such that $\beta k \varepsilon=1$. It follows from Lemma 7.3 that

$$
\begin{aligned}
& \int_{\operatorname{dist}(x, \Gamma)<1} \operatorname{dist}(x, \Gamma)^{-1} d \operatorname{Vol}(y) \\
= & \sum_{i=0}^{\infty} \int_{2^{-i-1}<\operatorname{dist}(x, \Gamma) \leq 2^{-i}} \operatorname{dist}(x, \Gamma)^{-1} d \operatorname{Vol}(y) \leq \sum_{i=0}^{\infty} \frac{1}{2^{2 i}} 2^{i+1}<\infty .
\end{aligned}
$$

Hence $\Omega$ is a $\psi$-regular domain, provided $\psi^{\prime}(x) \leq C e^{k x}$. Even if this is not true, let $\psi_{1}(x)=C\left(e^{k x}-1\right)$. Then $\psi \leq \psi_{1}$ for $C$ and $k$ sufficiently large. Domain $\Omega$ is $\psi_{1}$-regular and the large solution of $\Delta u-c \psi_{1}(u)=0$ in $\Omega$ is a subsolution of $\Delta u-c \psi(u)=0$ in $\Omega$. This implies that every boundary point has a local $(c \psi)$-barrier, so $\Omega$ is $\psi$-regular.

Proof of the claim in Example 3.2. Assume that $\Gamma$ satisfies (20). Then the following lemma holds.

Lemma 7.5. There exists $C=C\left(c_{1}, c_{2}\right)>0$ such that for all $0<r \leq s<1$ and $x \in \Gamma$ :

$$
H^{n}\left(\left\{x \in M: \operatorname{dist}\left(x, \Gamma_{s}\right)<r\right\}\right) \leq C r^{n-d} s^{d}, \quad \text { where } \Gamma_{s}=\Gamma \cap B(x, s) .
$$

The proof of the lemma is quite technical and is not related to the main purpose of this article, so we only sketch the most important points. The idea is that $H^{d}\left(\Gamma_{s}\right) \approx s^{d}$. Using Lemma 7.3 it follows that (123) holds for small $r>0$. On the other hand, the lemma is trivially true for $r \approx s$. The idea of the proof which allows us to show it for all $r$ is to cover the set $\Gamma_{s}$ by balls of radius $r$. Now we increase the radius of all balls to $k r, k=2,3, \ldots$. These $k r$ balls cover $\Gamma_{s}$ and they have quite a large overlap. For the lower bound $c_{1} r^{d}$ in (123) let us estimate how many fewer balls we need to have $\Gamma_{s}$ covered (we need only about $1 / k^{d}$ of the original number of balls). From this the estimate follows.

Now we continue with the proof of the example. Let $u$ be as in Lemma 7.1 . Also having the lower bound on the measure, we get that $u$ is comparable to $\operatorname{dist}(., \Gamma)^{-(n-d-2)}$. It follows that (115) certainly holds if

$$
\int_{\Omega} \frac{d(x, y)^{2-n}}{\operatorname{dist}(y, \Gamma)^{\alpha(n-d-2)}} d \operatorname{Vol}(y)=o\left(\operatorname{dist}(x, \Gamma)^{-(n-d-2)}\right), \quad \text { as } x \rightarrow \Gamma .
$$

Fix $x \in \Omega$ close to $\Gamma$ and let $\delta=\operatorname{dist}(x, \Gamma)>0$. The function we integrate has two singularities, one near $x$ and the other one near $\Gamma$. Therefore we introduce a bit complicated division of $\Omega$. Let $B_{0}=\{y \in \Omega$ : $\operatorname{dist}(y, \Gamma)<\delta / 2\}, B_{1}=\Omega \backslash B_{0}$. Clearly, $\int_{B_{1}} d(x, y)^{2-n} \operatorname{dist}(y, \Gamma)^{-\alpha(n-d-2)} d \operatorname{Vol}(y) \leq C \delta^{2-\alpha(n-d-2)}$. Let $z \in \Gamma$ be a point such that $\delta=\operatorname{dist}(x, z)$. Write $B_{0}$ as $\bigcup_{k=1}^{\infty} C_{k}$, where $C_{k}=\{x \in \Omega$ : $\left.\delta / 2^{k+1} \leq \operatorname{dist}(x, \Gamma)<\delta / 2^{k}\right\}$. Finally, let $D_{0}=\emptyset, D_{i}=\left\{y \in \Omega: \operatorname{dist}(z, y)<2^{i} \delta\right\}$, 
$i=1,2, \ldots$ and $E_{i}=D_{i} \backslash D_{i-1}$. If $y \in E_{i} \cap C_{k}$, then $\operatorname{dist}(y, \Gamma) \approx \delta / 2^{k}$ and $\operatorname{dist}(x, y) \approx 2^{i} \delta$. Also by (123),

$$
\operatorname{Vol}\left(E_{i} \cap C_{k}\right)=H^{n}\left(E_{i} \cap C_{k}\right) \leq C 2^{i} \delta^{d}\left(\delta / 2^{k}\right)^{n-d}=C \delta^{n} 2^{i d-k(n-d)} .
$$

It follows that

$$
\begin{aligned}
& \int_{E_{i} \cap C_{k}} \frac{d(x, y)^{2-n}}{\operatorname{dist}(y, \Gamma)^{\alpha(n-d-2)}} d \operatorname{Vol}(y) \\
\leq & C\left(2^{i} \delta\right)^{2-n}\left(2^{k} / \delta\right)^{\alpha(n-d-2)} \delta^{n} 2^{i d-k(n-d)} \\
\leq & C \delta^{2-\alpha(n-d-2)} 2^{i(d-n+2)+\alpha(n-d-2)-n+d)} .
\end{aligned}
$$

As $d-n+2<0$ and $\alpha(n-d-2)-n+d<0$ we can sum over $i$ and $k$ and get that (124) is less than $C \delta^{2-\alpha(n-d-2)}=o\left(\delta^{-(n-d-2)}\right)$ because $2-\alpha(n-d-2)>-(n-d-2)$. This concludes the proof.

\section{LOCALIZATION PRINCIPLE (UNIQUENESS)}

In this section we will prove theorems from section 3 concerning uniqueness. As with the existence, we see that the uniqueness is also a local property of points of $\partial \Omega$. This completes the picture and reduces both questions of existence and uniqueness of large solutions on a domain $\Omega$ to the study of the local properties of its boundary.

Proof of Theorem 3.4. We borrow the main idea from [42] but modify it. Let us assume that $u$ and $v$ are two comparable large solutions of the equation $\Delta u-\psi(u)=$ 0 in $\Omega$. If $u=v$ there is nothing to prove, so let us assume that $u \neq v$. It follows that $\max \{u, v\}$ is a subsolution and $u+v$ is a supersolution of the equation $\Delta u-\psi(u)=0$ in $\Omega$. Hence, as before, there is a solution $w$ of the equation $\Delta u-\psi(u)=0$ such that

$$
\max \{u, v\} \leq w \leq u+v
$$

Crucially, $u \leq w$ and $v \leq w$ everywhere in $\Omega$. If we prove that $u=w$, then we are done as the same argument would also give us that $v=w$, and hence $u=w=v$.

Notice also that $u$ and $w$ are also comparable. Let $K$ be a number for which (21) holds. Then any other large solution $u^{\prime}$ of the equation $\Delta u-\psi(u)=0$ in $\Omega$ comparable to $u$ (hence $w$ ) will satisfy

$$
w \leq K^{2} u^{\prime} \quad \text { in } \Omega .
$$

If $K=1$ we are done, so let us assume that $K>1$.

We claim that the function $v_{1}=u-\frac{1}{2 K^{2}}(w-u)$ is a supersolution of our equation that is greater than $\left(K^{2}+1\right) u /\left(2 K^{2}\right)$. Indeed,

$$
\begin{aligned}
& \Delta v_{1}-\psi\left(v_{1}\right)=\Delta v_{1}-\frac{2 K^{2}+1}{2 K^{2}} \frac{2 K^{2}}{2 K^{2}+1} \psi\left(\frac{2 K^{2}+1}{2 K^{2}} u-\frac{1}{2 K^{2}} w\right) \\
\leq & \Delta v_{1}-\frac{2 K^{2}+1}{2 K^{2}}\left[\psi(u)-\frac{1}{2 K^{2}+1} \psi(w)\right] \\
= & \frac{2 K^{2}+1}{2 K^{2}}(\Delta u-\psi(u))-\frac{1}{2 K^{2}}(\Delta w-\psi(w))=0 .
\end{aligned}
$$

On the other hand for any $\theta \in(0,1)$ we have that $v_{2}=\theta u$ is a large subsolution of our equation in $\Omega$. For any $\theta<\left(K^{2}+1\right) /\left(2 K^{2}\right)$ we may conclude that $v_{2} \leq v_{1}$ near $\partial \Omega$. Hence by the comparison principle, $v_{1} \leq v_{2}$ everywhere in $\Omega$. It follows that 
there is a large solution $u_{1}$ of the equation $\Delta u-\psi(u)=0$ such that $v_{1} \leq u_{1} \leq u_{2}$ in $\Omega$. We also have that

$$
u_{1} \leq v_{1}=u-\frac{1}{2 K^{2}}(w-u) \quad \text { in } \Omega .
$$

Hence

$$
w-u_{1} \geq\left(1+\frac{1}{2 K^{2}}\right)(w-u) .
$$

We now repeat the whole construction with $u_{1}$ in place of $u$ and obtain a large solution $u_{2}$ of our equation in $\Omega$ such that

$$
w-u_{2} \geq\left(1+\frac{1}{2 K^{2}}\right)\left(w-u_{1}\right) .
$$

Inductively we can define a decreasing sequence of large solutions $u, u_{1}, u_{2}, u_{3}, \ldots$ that all are comparable to $w$ and we will have

$$
w-u_{n} \geq\left(1+\frac{1}{2 K^{2}}\right)^{n}(w-u) .
$$

However, this is clearly impossible if $K>1$ as that would imply that $w(x)-u_{n}(x) \rightarrow$ $\infty$ for all $x \in \Omega$, where $u(x)<w(x)$. From this it follows that $K=1$; hence $u=w$.

Proof of Theorem 3.5. Pick $\theta>1$ for which (8) holds. It clearly suffices to show that at any point $x \in \Omega: u(x) / v(x) \leq \theta$ for two complete comparable large solutions $u$ and $v$, since that gives that $u(x) / v(x) \leq 1$ as the set of $\theta$ for which (8) holds has 1 as its limit point. From this by symmetry $u=v$ in $\Omega$. Moreover, it suffices to reduce our attention to points $x$ close to the boundary of $\Omega$ where Lemma 4.8 can be applied.

Since $u$ and $v$ are comparable, $u / v \leq R_{\max }<\infty$. If $R_{\max } \leq \theta$ we are done. Otherwise let us define functions $g$ and $R$ as follows:

$$
\begin{aligned}
g(r) & =\inf \{\varphi(v(x)): x \in \Omega \text { and } \delta(x) \leq r\}, \\
R(r) & =\sup \left\{\frac{u(x)}{v(x)}: x \in \Omega \text { and } \delta(x) \geq r\right\} .
\end{aligned}
$$

Function $g$ is nonincreasing and $g(r) \geq 1 /\left(r^{2} f(r)\right)$. Similarly, $R$ is nonincreasing (comparison lemma) at points where $R>1$. For a point $x \in \Omega$, let $\delta=\delta(x) / 2$ be as in Lemma 4.8 If $B=B(x, \delta)$, then

$$
\inf _{z \in B} \varphi(u(x)) \geq g(3 \delta) \geq \frac{1}{9 \delta^{2} f(3 \delta)},
$$

since all points in $B$ have distance at most $3 \delta$ from the boundary. With $R=R(\delta)$ used in Lemma 4.8 we have that $\Theta v$ is a supersolution in $B$ and $\left.\Theta v\right|_{\partial B} \geq\left. u\right|_{\partial B}$ as on $\partial B: u(x) / v(x) \leq R(\delta)$ (no point of $B$ is closer than $\delta$ from the boundary). Thus by the comparison lemma, $u \leq \Theta v$ in $B$. In particular, at $x, u(x) / v(x) \leq \Theta(0)$. If $\Theta(0) \leq \theta$ we are done and there is nothing else to prove. Otherwise,

$$
\frac{u(x)}{v(x)} \leq R(\delta)-C\left(\inf _{z \in B} \varphi(u(x))\right) \delta^{2} \leq R(\delta)-\frac{C}{9 f(3 \delta)},
$$


where $C>0$ is the same constant as in (65). As $x$ is an arbitrary point of distance $2 \delta$ from the boundary, taking the supremum over all such points gives us

$$
R(2 \delta) \leq R(\delta)-C\left(\inf _{z \in B} \varphi(u(x))\right) \delta^{2} \leq R(\delta)-\frac{C_{0}}{f(3 \delta)}
$$

for a new constant $C_{0}>0$. Here we use the fact that the supremum over such points equals $R(2 \delta)$, as $R$ is a nonincreasing function.

The inequality (131) can be iterated, as many times as we want, provided the right hand side of it is greater than $\theta$. We just replace $\delta$ by $\delta / 2$, etc. This yields

$$
R(2 \delta) \leq R\left(2 \delta / 2^{n}\right)-\sum_{k=1}^{n} \frac{C_{0}}{f\left(6 \delta / 2^{k}\right)}, \quad \text { for } n=1,2, \ldots
$$

If the sum in (132) diverges as $n \rightarrow \infty$, then for some finite $n$ the right hand side must be less than $\theta$, as $R\left(2 \delta / 2^{n}\right) \rightarrow R_{\max }<\infty$. From this the claim $u(x) / v(x) \leq \theta$ follows. The sum is indeed divergent as it is comparable with $\int_{0}^{\varepsilon} d t /(t f(t))$.

Remark 8.1. It follows from the previous proof that the argument works even if the solutions $u$ and $v$ are not comparable as long as the sum in (132) grows faster to infinity than $R\left(2 \delta / 2^{n}\right)$ as $n \rightarrow \infty$. In reality, this brings only a very minor improvement, as it allows the ratio $u(x) / v(x)$ to grow at the rate of at most $C|\ln \delta(x)|$ for $\delta(x) \rightarrow 0$ or worse (double logarithm). For this reason we do not formulate this observation in a theorem.

Corollary 8.1. Let the function $\psi$ satisfy the standard assumptions. If a solution $u$ of the equation $\Delta u-\psi(u)=0$ in $\Omega$ blows up at the maximal rate (Definition 2.5), then $u$ is the maximal solution $U_{\max }$ defined in Theorem 3.2 .

We start with a technical lemma, introducing once again solutions as in (iv) of Proposition 6.2 i.e., solutions that blow up on part of the boundary and vanish on the other part.

Lemma 8.2. Let $\Omega$ be a $\psi$-regular domain. Consider a neighborhood $U$ of $x \in \partial \Omega$ with $C^{1}$ boundary. Then for any solution $u$ of the equation $\Delta u-\psi(u)=0$ in $U \cap \Omega$ such that $u(y) \rightarrow \infty$ locally uniformly as $y \rightarrow \partial \Omega \cap U$ there exists a nonnegative solution $u_{0}$ of $\Delta u-\psi(u)=0$ in $U \cap \Omega$ such that:

(a) $u_{0}(y) \rightarrow \infty$ as $y \rightarrow \Gamma_{1}=\partial \Omega \cap U$.

(b) $u_{0}$ is continuous in $\Omega \cup \Gamma_{2}$ and $u_{0}=0$ on $\Gamma_{2}=\Omega \cap \partial U$.

Moreover, $u_{0}<u<u_{0}+v_{\max }$, where $v_{\max }$ is the large solution of $\Delta u-\psi(u)=0$ in $U$.

Proof of Lemma 8.2. This proof requires a slightly more refined argument than the construction given in Proposition 6.2. Let $V_{1} \Subset V_{2} \Subset \cdots \Subset U$ be an increasing sequence of $C^{1}$ domain whose union is $U$. Let us denote by $v_{n}$ the large solution of our equation on $V_{n}$. Notice that $v_{\max }=\lim _{n \rightarrow \infty} v_{n}$. For each $n \in \mathbb{N}$, let $\Gamma_{n}^{1}=$ $\partial V_{n} \cap\{y \in \Omega: \operatorname{dist}(y, \partial \Omega)>1 / n\}$ and $\Gamma_{n}^{2}=\partial V_{n} \backslash\{y \in \Omega: \operatorname{dist}(y, \partial \Omega) \geq 1 / n\}$. Both sets are open and also disjoint. It follows (by refining the argument of Theorem 5.3) that given any two bounded and continuous functions $g_{1}$ on $\Gamma_{n}^{1}$ and $g_{2}$ on $\Gamma_{n}^{2}$ we can find a solution of $\Delta u-\psi(u)=0$ on $V_{n}$ which is continuous on $V_{n} \cup \Gamma_{n}^{1} \cup \Gamma_{n}^{2}$ and attains boundary values given by $g_{1}, g_{2}$, respectively. We let $V_{n}$ be zero on the remaining portion of the boundary. This might cause discontinuities at the ends. 
Let $u$ be any solution in $U \cap \Omega$ satisfying the assumption. Let $g_{1}=\left.u\right|_{\Gamma_{n}^{1}}$ and $g_{2}=0$ on $\Gamma_{n}^{2}$. It follows that $g_{1}$ is bounded and continuous; hence there is a solution $\widetilde{v_{n}}$ in $V_{n}$ such that $\left.\widetilde{v_{n}}\right|_{\Gamma_{n}^{1}}=\left.u\right|_{\Gamma_{n}^{1}}$ and $\left.\widetilde{v_{n}}\right|_{\Gamma_{n}^{2}}=0$. By the comparison lemma it follows that $\widetilde{v_{n}} \leq u$ in $V_{n} \cap \Omega$. Hence $w_{n}=u-\widetilde{v_{n}}$ is a nonnegative subsolution of our equation in $V_{n} \cap \Omega$ such that $w_{n}$ vanishes on $\Gamma_{n}^{1}$ and blows up as it approaches $\partial \Omega \cap V_{n}$. Let $U_{0}$ be the solution constructed in Lemma 6.1 for $U \cap \Omega$. It follows from the construction that $w_{n} \leq U_{0}$. It also follows that $w_{n}$ belongs to the set

$$
\begin{aligned}
\mathcal{S}_{n}=\left\{f: V_{n} \cap \Omega \rightarrow \mathbb{R}^{+}:\right. & f \text { is a subsolution of } \Delta u-\psi(u)=0 \\
& \text { in } \left.V_{n} \cap \Omega \text { such that } f \leq \min \left\{u, U_{0}\right\}\right\} .
\end{aligned}
$$

Let $u_{n}(y)=\sup _{f \in \mathcal{S}_{n}} f(y)$. Standard elliptic arguments imply that $u_{n}$ is well defined as $\mathcal{S}_{n}$ is nonempty and it solves our equation in $V_{n} \cap \Omega$. Also since $\left.\mathcal{S}_{n+1}\right|_{V_{n}} \subset \mathcal{S}_{n}$, the sequence $u_{n}$ decreases monotonely. Let $u_{0}=\lim _{n \rightarrow \infty} u_{n}$. It follows that $u_{0}$ is also well defined and is a solution of the equation in $U \cap \Omega$. The definition also implies that $u_{0} \leq u_{n} \leq \min \left\{u, U_{0}\right\}$; hence $u_{0}(y) \rightarrow 0$ locally uniformly as $y \rightarrow \Gamma^{2}$ and $u_{0} \leq u$. On the other hand, $u_{n} \geq w_{n}=u-\widetilde{v_{n}}$. Taking the limit yields $u_{0} \geq u-v_{\max }$, which implies that the desired inequality follows, as well as that $u_{0}(y) \rightarrow \infty$ locally uniformly as $y \rightarrow \Gamma^{1}$. The claim that these inequalities are in fact strict is a consequence of the strong maximum principle.

Proof Theorem 3.6. It suffices to prove the only if part. We first assume that part (a) of Definition 2.11 holds. We consider only the case $c=1$ as the rest is similar.

Let $u^{1}, u^{2}$ be any two large solutions in $\Omega$. Pick a point $x \in \partial \Omega$ and denote by $V_{x}$ the neighborhood from Definition 2.11 such that (15) holds. By $U_{x}$ we denote a neighborhood of $x$ with $C^{1}$ boundary such that $U_{x} \subset V_{x}$. If $\Omega \subset V_{x}$ it follows immediately that (136) holds and we skip all that follows. Otherwise, consider $u^{1}$, $u^{2}$ restricted to $U_{x}$. By the previous lemma there are $u_{0}^{1}$ and $u_{0}^{2}$ defined on $U_{x} \cap \Omega$ satisfying (a), (b) such that $u_{0}^{1} \leq u^{1} \leq u_{0}^{1}+v_{\max }, u_{0}^{2} \leq u^{2} \leq u_{0}^{2}+v_{\max }$, where $v_{\max }$ is the largest solution on $U_{x}$. It follows that

$$
\lim _{y \rightarrow z} \frac{u^{1}(y)}{u_{0}^{1}(y)}=\lim _{y \rightarrow z} \frac{u^{2}(y)}{u_{0}^{2}(y)}=1, \quad \text { for all } z \in \partial \Omega \cap U_{x} .
$$

Now extend both $u_{0}^{1}$ and $u_{0}^{2}$ onto $V_{x} \cap \Omega$ by putting $u_{0}^{1}=u_{0}^{2}=0$ outside $U_{x}$. It follows that this extension defines subsolutions on $V_{x} \cap \Omega$. On the other hand, if $u$ is any large solution on $V_{x}$, then $u^{1}+u$ and $u^{2}+u$ are large supersolutions on $V_{x} \cap \Omega$. Thus for any $\varepsilon>0$ small, $(1-\varepsilon) u_{0}^{1}+\varepsilon u \leq u^{1}+u,(1-\varepsilon) u_{0}^{2}+\varepsilon u \leq u^{2}+u$ on $V_{k} \cap \Omega$ and $(1-\varepsilon) u_{0}^{1}+\varepsilon u,(1-\varepsilon) u_{0}^{2}+\varepsilon u$ are large positive subsolutions; $u^{1}+u, u^{2}+u$ are large positive supersolutions. By a standard argument it follows that there are large solutions $v^{1}, v^{2}$ on $V_{x} \cap \Omega$ such that $(1-\varepsilon) u_{0}^{i}+\varepsilon u \leq v^{i} \leq u^{i}+u$ for $i=1,2$. The assumption that the point $u_{x}$ is $\psi$-unique on $V_{x}$ implies that $\lim _{y \rightarrow x} \frac{v^{1}(y)}{v^{2}(y)}=1$. From this it follows that

$$
1-\varepsilon \leq \liminf _{y \rightarrow x} \frac{u^{1}(y)}{u^{2}(y)} \leq \limsup _{y \rightarrow x} \frac{u^{1}(y)}{u^{2}(y)} \leq \frac{1}{1-\varepsilon} .
$$

As the $\varepsilon$ can be taken to be arbitrarily small, we get that

$$
\lim _{y \rightarrow x} \frac{u^{1}(y)}{u^{2}(y)}=1
$$


Now as $x$ was an arbitrary point at the boundary and $\partial \Omega$ is a compact set, it follows that $u^{1}(y) / u^{2}(y) \rightarrow 1$ uniformly as $y \rightarrow \partial \Omega$. Consequently, for every $\varepsilon>0$ there exists a neighborhood $\Omega_{\delta}=\{x \in \Omega: \operatorname{dist}(x, \partial \Omega)<\delta\}$ of the boundary where $u^{1} \leq(1+\varepsilon) u^{2}$. Hence, by the comparison lemma we get $u^{1} \leq(1+\varepsilon) u^{2}$ in $\Omega \backslash \Omega_{\delta}$ as this inequality holds on the boundary of this domain. So, $u^{1} \leq(1+\varepsilon) u^{2}$ in $\Omega$, i.e., $u^{1} \leq u^{2}$. By symmetry, $u^{2} \leq u^{1}$ and the uniqueness follows.

If part (b) of Definition 2.11 holds, then a small modification of the proof is required. By following the proof given above we obtain only that

$$
\limsup _{y \rightarrow \partial \Omega} \frac{u^{c}(y)}{u^{2}(y)}<\infty
$$

Hence two large solutions $u^{c}$ and $u^{2}$ are comparable. This together with condition $\lim \sup _{u \rightarrow \infty} \frac{\psi(\theta u)}{\psi(u)}<\infty$ implies that $u^{2}$ is also complete. So, $u^{c}=u^{2}$.

The following proposition shows that the neighborhood $U$ in Definition 2.11 can be taken to be "nice" (i.e. with $C^{1}$ boundary) and arbitrarily small.

Proposition 8.3. Suppose that function $\psi$ satisfies the Harnack assumptions. Let the domain $\Omega$ be $\psi$-unique. Then for any $x \in \partial \Omega$ and any open neighborhood $U$ of $x$ with $C^{1}$ boundary, (15) holds for any large solutions $u^{1}, u^{2}$ in $U \cap \Omega$.

Proof. Clearly $U^{c}=M \backslash \bar{U}$ has $C^{1}$ boundary. Thus there exists a large solution $u^{3}$ of $\Delta u-c \psi(u)=0$ on $U^{c} \cap \Omega$. By Lemma 8.2 we can find solutions $u_{0}^{1}, u_{0}^{2}$ in $U \cap \Omega$ and $u_{0}^{3}$ in $U^{c} \cap \Omega$ satisfying (a), (b) such that $u_{0}^{i} \leq u^{i} \leq u_{0}^{i}+w^{i}, i=1,2,3$. Here $w^{1}=w^{2}$ is a largest solution on $U$ and $w^{3}$ on $U^{c}$.

Finally, consider $v_{i}, i=1,2$, defined on the whole $\Omega$ by $v^{i}=u_{0}^{i}$ on $\bar{U} \cap \Omega$ and $v^{i}=u_{0}^{3}$ on $U^{c} \cap \Omega, i=1,2$. Clearly, the $v^{i}$ are continuous functions on $\Omega$, locally Lipschitz and solve $\Delta u-c \psi(u)=0$ everywhere except on $\partial U \cap \Omega$. What happens locally at such a point? Recall the definition of the subsolution in (24). Let $V$ be a small neighborhood of $x \in \partial U \cap \Omega$. Then (24) simplifies to

$$
\int_{V}\left(\nabla v^{i} \nabla w+c \psi\left(v^{i}\right) w\right) d \mathrm{Vol} \leq 0 \quad \text { for any } w \geq 0 .
$$

The integral in (137) is well defined as $\nabla v^{i}$ is Lipschitz on $V$ and moreover $V$ can be split into two parts $V_{1}=V \cap U$ and $V_{2}=V \cap U^{c}$. Integrating by parts we get

$$
\begin{aligned}
& \int_{V}\left(\nabla v^{i} \nabla w+c \psi\left(v^{i}\right) w\right) d \mathrm{Vol}=\int_{V_{1}}\left(-\Delta u_{0}^{i}+c \psi\left(u_{0}^{i}\right)\right) w d \mathrm{Vol} \\
& +\int_{V_{2}}\left(-\Delta u_{0}^{3}+c \psi\left(u_{0}^{3}\right)\right) w d \mathrm{Vol}+\int_{\partial U \cap V}\left(\frac{\partial u_{0}^{i}}{\partial \nu}-\frac{\partial u_{0}^{3}}{\partial \nu}\right) w d S \\
& =\int_{\partial U \cap V}\left(\frac{\partial u_{0}^{i}}{\partial \nu}-\frac{\partial u_{0}^{3}}{\partial \nu}\right) w d S .
\end{aligned}
$$

Here $\nu$ is the normal to $\partial U \cap V$ pointing into $V_{2}$. As both $u_{0}^{i}$ and $u_{0}^{3}$ are positive outside $\partial U \cap V$ and zero there we conclude that $\frac{\partial u_{0}^{i}}{\partial \nu} \leq 0$ and $\frac{\partial u_{0}^{3}}{\partial \nu} \geq 0$; i.e. $v^{i}$ is a subsolution. Using a partition of unity it follows that $v^{i}$ is a subsolution on the whole $\Omega$. Finally, for any $\varepsilon>0$ let $W_{\varepsilon}$ be a domain in $M$ with $C^{1}$ boundary such that $\{y \in M: \operatorname{dist}(y, \partial \Omega \cap \partial U) \leq \varepsilon / 2\} \subset M \backslash W_{\varepsilon} \subset\{y \in M: \operatorname{dist}(y, \partial \Omega \cap \partial U) \leq \varepsilon\}$ 
and let $w_{\varepsilon}$ be a large solution on $W_{\varepsilon}$. For $i=1,2$ consider

$$
\begin{aligned}
\mathcal{S}_{\varepsilon}^{i}=\left\{f: W_{\varepsilon} \cap \Omega \rightarrow \mathbb{R}^{+}: \quad\right. & f \text { is a subsolution of } \Delta u-\psi(u)=0 \\
& \text { in } W_{\varepsilon} \cap \Omega \text { such that } f \leq u^{i}+w_{\varepsilon} \\
& \text { on } \left.U \cap W_{\varepsilon} ; f \leq u^{3}+w_{\varepsilon} \text { on } U^{c} \cap W_{\varepsilon}\right\} .
\end{aligned}
$$

The sets $S_{\varepsilon}^{i}$ are nonempty as $\max \left\{v^{i}, w_{\varepsilon}\right\}$ is a subsolution in $S^{i}$. It follows that $U_{\varepsilon}^{i}(y)=\sup _{f \in \mathcal{S}_{\varepsilon}^{i}} f(y)$ for $y \in W_{\varepsilon} \cap \Omega$ is well defined and (by a standard argument) solves our equation. Clearly as $U_{\varepsilon}^{i} \geq v^{i}$ and $U_{\varepsilon}^{i} \geq w_{\varepsilon}$, hence $U_{\varepsilon}^{i} \rightarrow \infty$ uniformly on $\partial\left(\Omega \cap W_{\varepsilon}\right)$; i.e. $U_{\varepsilon}^{i}$ is a large solution on $W_{\varepsilon} \cap \Omega$. Finally, sets $W_{\varepsilon}$ can be chosen such that if $0<\varepsilon_{1}<\varepsilon_{2}$, then $\overline{W_{\varepsilon_{2}}} \subset W_{\varepsilon_{1}}$ leading to $\left.S_{\varepsilon_{1}}^{i}\right|_{W_{\varepsilon_{2}}} \subset S_{\varepsilon_{2}}^{i}$, i.e., $U_{\varepsilon_{1}}^{i} \leq U_{\varepsilon_{2}}^{i}$. Hence, limiting in $\varepsilon \rightarrow 0+$ we get solutions $U^{i}$ on the whole $\Omega$ such that $u_{0}^{i}=\left.v^{i}\right|_{U \cap \Omega} \leq U^{i} \leq u^{i}+w \leq u_{0}^{i}+2 w^{i}$ on $\Omega \cap U$ and $u_{0}^{3}=\left.v^{i}\right|_{U^{c} \cap \Omega} \leq U^{i} \leq u^{3}+w \leq$ $u_{0}^{3}+2 w^{3}$ on $U^{c} \cap \Omega$ for $i=1,2$. Here $w=\lim _{\varepsilon \rightarrow 0+} w_{\varepsilon}$, and $w \leq w^{i}, i=1,2,3$ as $U, U^{c} \subset \bigcup W_{\varepsilon}$. These estimates show that $U^{i}, i=1,2$ are large solutions on $\Omega$. Since $\Omega$ is $\psi$-unique it follows that $U^{1}=U^{2}$. Hence on $U \cap \Omega$ we have that

$$
\frac{u^{1}(y)}{u^{2}(y)} \leq \frac{\left(u_{0}^{1}+2 w^{1}\right)(y)}{u_{0}^{2}(y)} \leq \frac{\left(U^{1}+2 w^{1}\right)(y)}{\left(U^{2}-2 w^{2}\right)(y)} \rightarrow 1
$$

uniformly for $y$ converging to any compact subset of $\partial \Omega \cap U$ as $U^{1}=U^{2} \rightarrow \infty$ and $w^{1}=w^{2}$ stays bounded. Switching roles of $u^{1}$ and $u^{2}$ yields a similar estimate for $u^{2} / u^{1}$. From this (15) follows.

Proof of Theorem 3.7. The existence of a large solution of (22) follows from previous results. Let $u$ be any such solution. Since $u(x) \rightarrow \infty$ as $x \rightarrow \partial \Omega$, and also $\psi(u) / u \rightarrow \infty$ it follows that for any $\varepsilon>0, u$ satisfies

$$
k(x)(1-\varepsilon) \psi(u(x)) \leq \Delta u(x) \leq k(x)(1+\varepsilon) \psi(u(x))
$$

on an open set $\Omega_{\delta}=\{x \in \Omega: \operatorname{dist}(x, \partial \Omega)<\delta\}$ for some $\delta=\delta(\varepsilon)>0$. The continuity of $k$ at the boundary of $\partial \Omega$ implies that there is $\delta^{\prime}>0$ such that the following holds for any $\varepsilon>0$ :

$$
\forall x \in \Omega, y \in \partial \Omega \text { such that } \operatorname{dist}(x, y)<\delta^{\prime} \Longrightarrow|k(x)-k(y)|<\varepsilon .
$$

It follows that (by possibly making $\delta>0$ smaller) we have

$$
k(y)(1-\varepsilon) \psi(u(x)) \leq \Delta u(x) \leq k(y)(1+\varepsilon) \psi(u(x))
$$

for all $x \in \Omega_{\delta}$ and $y \in \partial \Omega$ such that $\operatorname{dist}(x, y)<\delta$. Hence by Theorem 3.6 and Proposition 8.3 every boundary point $y \in \partial \Omega$ has a neighborhood $U_{y} \subset\{x \in M$ : $\operatorname{dist}(x, y)<\delta\}$ with $C^{1}$ boundary such that (15) holds for any large solutions of $\Delta u-\psi(u)=0$ in $U_{y} \cap \Omega$. Let $u_{y}$ be the largest solution of the equation

$$
\Delta u_{y}-k(y) \psi\left(u_{y}\right)=0 \quad \text { in } U_{y} \cap \Omega .
$$

We would like to know how this solution compares with large solutions of equations $\Delta u-k(y)(1-\varepsilon) \psi(u)=0$ and $\Delta u-k(y)(1+\varepsilon) \psi(u)=0$ in $U_{y} \cap \Omega$. For any $c \in(0, \infty)$ let $u^{c}$ be the largest solution of the equation

$$
\Delta u^{c}-c k(y) \psi\left(u^{c}\right)=0 \quad \text { in } U_{y} \cap \Omega .
$$

We have that $u_{y}=u^{1}$. If $c>1$, then $u^{1}$ is a supersolution of (145) and hence $u^{c} \leq u^{1}$. Similarly for $c<1$ we get $u^{1} \leq u^{c}$. Consider now any $\theta$ for which (8) 
holds. Recall that $\theta>1$. It follows from (7.11) that there exist $c_{0}>1$ and $M>0$ such that $\psi(\theta u) \geq c_{0} \theta \psi(u)$. Hence

$$
0=\Delta\left(\theta u^{1}\right)-\theta \psi\left(u^{1}\right) \geq \Delta\left(\theta u^{1}\right)-\frac{1}{c_{0}} \psi\left(\theta u^{1}\right) \geq \Delta\left(\theta u^{1}\right)-c \psi\left(\theta u^{1}\right)
$$

as long as $c>\frac{1}{c_{0}}$ and $u^{1} \geq M$ everywhere. The second can be arranged easily: just take $\delta>0$ in the definition of $\Omega_{\delta}$ sufficiently small. Having that, we get that $\theta u^{1}$ is a supersolution and $u^{1} \leq u^{c} \leq \theta u^{1}$ for any $c \in\left[\frac{1}{c_{0}}, 1\right]$. The same argument also yields $\frac{1}{\theta} u^{1} \leq u^{c} \leq u^{1}$ for $c \in\left[1, c_{0}\right]$. Hence for all $c \in\left[\frac{1}{c_{0}}, c_{0}\right]$ we have

$$
\frac{1}{\theta} \leq \liminf _{x \rightarrow y} \frac{u^{c}(x)}{u^{1}(x)} \leq \limsup _{x \rightarrow y} \frac{u^{c}(x)}{u^{1}(x)} \leq \theta .
$$

Recall that $\theta>1$ can be chosen arbitrarily close to 1 . Next, $\theta$ gives us $c_{0}$ and $M$. We pick $\varepsilon>0$ such that $1-\varepsilon, 1+\varepsilon \in\left[\frac{1}{c_{0}}, c_{0}\right]$ and $\delta>0$ such that (143) holds and also $u_{y}=u^{1}$ is larger than $\theta M$. This gives us that (147) holds for $c=1-\varepsilon$ and $c=1+\varepsilon$.

We return to (143). We see that $u$ is a subsolution of the equation (145) for $c=1-\varepsilon$. Therefore its restriction to $U_{y} \cap \Omega$ belongs to the set

$$
\mathcal{S}=\left\{f: \Omega \rightarrow \mathbb{R}^{+}: f \text { is a subsolution of } \Delta u-(1-\varepsilon) k(y) \psi(u)=0 \text { in } U_{y} \cap \Omega\right\} .
$$

As before $w(x)=\sup _{f \in \mathcal{S}} f(x)$ defined on $U_{y} \cap \Omega$ is a solution of $\Delta u-(1-$ $\varepsilon) k(y) \psi(u)=0$ in $U_{y} \cap \Omega$. As $u^{1-\varepsilon}$ is the largest solution it follows that $u \leq$ $w \leq u^{1-\varepsilon}$. A similar consideration for $u^{1+\varepsilon}$ implies that $u^{1+\varepsilon}-v \leq u$, where $v$ is the large solution of our equation for $c=1+\varepsilon$ on $U_{y}$. Combining these two facts with (147) finally yields

$$
\frac{1}{\theta} \leq \liminf _{x \rightarrow y} \frac{u(x)}{u_{y}(x)} \leq \limsup _{x \rightarrow y} \frac{u(x)}{u_{y}(x)} \leq \theta .
$$

Recall that $u$ was an arbitrarily large solution of (22) on $\Omega$. Assume that there are two such large solutions, say $u^{1}$ and $u^{2}$. (149) is a local statement that holds on $\Omega \cap U_{y}$ for both $u^{1}$ and $u^{2}$. However, as the boundary $\partial \Omega$ is compact and (149) holds for every boundary point, we get that the ratio $u^{1}(x) / u^{2}(x)$ as $x \rightarrow \partial \Omega$ can be estimated uniformly and

$$
\frac{1}{\theta^{2}} \leq \liminf _{x \rightarrow \partial \Omega} \frac{u^{1}(x)}{u^{2}(x)} \leq \limsup _{x \rightarrow \partial \Omega} \frac{u^{1}(x)}{u^{2}(x)} \leq \theta^{2} .
$$

Finally, as $\theta>1$ can be chosen arbitrarily close to 1 we have that

$$
\lim _{x \rightarrow \partial \Omega} \frac{u^{1}(x)}{u^{2}(x)}=1 \quad \text { uniformly. }
$$

Proof of Theorem 3.8. Pick $V \Subset U$ to be an open $C^{1}$ neighborhood of $x$. Consider two large solutions $u_{1}, u_{2}$ of $\Delta u-\psi(u)=0$ in $\Omega$. Let $W$ be one open connected component of $U \cap \Omega$ and $\Phi_{t}$ the corresponding isometries. Let $v$ be the largest solution on $V$. It follows that for $t>0$ small, $\Phi_{t}(\bar{V}) \subset U$. Hence $\Phi_{t}\left(u_{1}\right)$ solves $\Delta u-\psi(u)=0$ in $\Phi_{t}(V \cap W) \Subset U \cap \Omega$ and $\Phi_{t}\left(u_{1}\right)+v$ is a large supersolution in $V \cap \Phi_{t}(V \cap W)$. The other function $u_{2}$ is bounded on this set; hence by the comparison lemma $u_{2} \leq \Phi_{t}\left(u_{1}\right)+v$. Letting $t \rightarrow 0+$ gives us $u_{2} \leq u_{1}+v$ in $V \cap W$. As this holds on each component of $U \cap \Omega$ we get that $u_{2} \leq u_{1}+v$ in 
$U \cap \Omega$. Switching the roles of $u_{1}$ and $u_{2}$ we obtain the other inequality; hence $u_{1}-v \leq u_{2} \leq u_{1}+v$. From this, $u_{1}(y) / u_{2}(y) \rightarrow 1$ as $y \rightarrow \partial \Omega \cap B(x, \delta)$ for some small $\delta>0$, so $x$ is $\psi$-unique.

\section{Applications to specific CASEs of $\psi$-Regular AND $\psi$-Unique domains}

Up to this point we considered the general theory of existence and uniqueness of large solutions on domains $\Omega$. We developed concepts of $\psi$-regularity and $\psi$ uniqueness. In this section we want to present how the developed theory can be used in specific cases that are interesting for some reasons. The presented results are not necessarily new; the point is to show how they nicely fit into our unified framework.

Our first observation is that in many situations we only need to consider the flat case, i.e., $\mathbb{R}^{n}$.

Proposition 9.1. Let $\Omega \subset M$ be an arbitrary domain. Assume that $\psi$ satisfies (6) and (8) for some $\theta>1$. Let $g, g^{\prime}$ be two $C^{2}$ Riemannian metric tensors on $M$ such that $\left\|g-g^{\prime}\right\|_{L i p(M)}<\varepsilon$ and let $u$ be a large solution of the equation $\Delta_{g} u-\psi(u)=0$ in $\Omega$. Assume that one of the following holds:

(a) $u$ has a maximal rate of blowup.

(b) $u$ satisfies $\left|\nabla^{2} u\right| \approx \psi(u)$ and $|\nabla u| \leq C \psi(u)$.

(c) $u$ satisfies $|\nabla u| \leq C \psi(u)$ and $\left.g\right|_{\partial \Omega}=\left.g^{\prime}\right|_{\partial \Omega}$.

(d) $\left.g\right|_{\partial \Omega}=\left.g^{\prime}\right|_{\partial \Omega}$ and $\left.\nabla g\right|_{\partial \Omega}=\left.\nabla g^{\prime}\right|_{\partial \Omega}$.

Then if $\varepsilon>0$ is sufficiently small the equation $\Delta_{g^{\prime}} v-\psi(v)=0$ in $\Omega$ has a large solution comparable to $u$, i.e., $u \approx v$.

Proof. Given two metric tensors $g$ and $g^{\prime}$, their action on a function $u$ can be bounded by

$$
\begin{aligned}
\left|\left(\Delta_{g}-\Delta_{g^{\prime}}\right) u\right| & \leq C\left\|g-g^{\prime}\right\|_{L^{\infty}(\Omega)}\left|\nabla^{2} u\right|+C\left\|g-g^{\prime}\right\|_{L i p(\Omega)}|\nabla u| \\
& \leq C \varepsilon\left(\left|\nabla^{2} u\right|+|\nabla u|\right),
\end{aligned}
$$

as follows from (43). The discussion we had in (145)-(147) implies that for any $c \in \mathbb{R}^{+}$the equation $\Delta_{g} u-c \psi(u)=0$ has a solution $u^{c}$ comparable to $u$. A similar statement is true for the equation $\Delta_{g^{\prime}} v-c \psi(v)=0$ whose solution $v^{c}$ is comparable to $v=v^{1}$. Of course, we first have to establish that $v$ exists.

We will show that $u$ is a subsolution of the equation $\Delta_{g^{\prime}} v-c \psi(v)=0$ for some $c<1$ small and that $u$ is a supersolution of the same equation for $c>1$ large. This is enough to see that the equation $\Delta_{g^{\prime}} v-c \psi(v)=0$ has large solutions $v^{c}$ in $\Omega$ for all $c$ and they are comparable to $u$. Indeed, it is enough to show this for $v=v^{1}$. But since $u$ is a subsolution of $\Delta_{g^{\prime}} v-c \psi(v)=0$ for some $c<1$ it follows that $u / \theta$ for $\theta \gg 1$ is a subsolution of $\Delta_{g^{\prime}} v-\psi(v)=0$. Similarly, since $u$ is a supersolution of the equation $\Delta_{g^{\prime}} v-c \psi(v)=0$ for some $c>1$, then $\theta u$ for $\theta \gg 1$ is a supersolution of the equation $\Delta_{g^{\prime}} v-\psi(v)=0$. From this $u / \theta \leq v^{1} \leq u \theta$ and the claim follows.

First assume that (a) holds. Then $\varphi(u) \approx \delta^{-2}$, hence $\psi(u) \approx u / \delta^{2}$. Hence by (64), $\left|\nabla^{2} u\right| \leq C u / \delta^{2} \approx \psi(u)$. On the other hand $C\left|\nabla^{2} u\right| \geq\left|\Delta_{g} u\right|=\psi(u)$ from which $\left|\nabla^{2} u\right| \approx \psi(u)$. We also get the estimate $|\nabla u| \leq C \delta \psi(u)$. Hence (b) holds. Having (b) and (152) implies that

$$
\left|\left(\Delta_{g}-\Delta_{g^{\prime}}\right) u\right| \leq C \varepsilon \psi(u) .
$$


From this we immediately get that

$(1-C \varepsilon) \psi(u) \leq \Delta_{g^{\prime}} u-\left|\left(\Delta_{g}-\Delta_{g^{\prime}}\right) u\right| \leq \Delta_{g^{\prime}} u \leq \Delta_{g^{\prime}} u+\left|\left(\Delta_{g}-\Delta_{g^{\prime}}\right) u\right| \leq(1+C \varepsilon) \psi(u)$.

Hence if $C \varepsilon<1$ the claim follows.

If (c) holds we in addition obtain that $\left|\left(g-g^{\prime}\right)(x)\right| \leq \varepsilon \delta(x)$. A variant of (64) ( $\nabla u$ satisfies a similar equation as $u$ itself) implies that $\delta\left|\nabla^{2} u\right| \leq C|\nabla u|$ which together with the above estimate yields

$$
\left|\left(\Delta_{g}-\Delta_{g^{\prime}}\right) u\right| \leq C \varepsilon \delta\left(\delta^{-1}\right)|\nabla u|+C \varepsilon|\nabla u| \leq C \varepsilon \psi(u) .
$$

From this we again get (154).

Finally, if (d) holds, we also assume that $\left\|g-g^{\prime}\right\|_{C^{2}(M)}<\varepsilon$. Then $\left|\left(g-g^{\prime}\right)(x)\right|$ $\leq \varepsilon \delta^{2}(x)$ and $\left|\nabla\left(g-g^{\prime}\right)(x)\right| \leq \varepsilon \delta(x)$. Using this and (64) in (152) yields

$$
\left|\left(\Delta_{g}-\Delta_{g^{\prime}}\right) u\right| \leq C \varepsilon \delta^{2}\left(\delta^{-2} u\right) u+C \varepsilon \delta\left(\delta^{-1} u\right) \leq C \varepsilon u \leq C \varepsilon \psi(u) .
$$

Again (154) follows. Now we can remove the assumption of "smallness" of the $C^{2}$ norm of the difference $g-g^{\prime}$ by using the fact that any two metrics that satisfy (d) can be connected by a continuous curve $\gamma:[0,1] \rightarrow \mathcal{G}$, where $\mathcal{G}$ is a space of $C^{2}$ metrics satisfying $(\mathrm{d}), \gamma(0)=g$ and $\gamma(1)=g^{\prime}$. Our result implies that every $t \in[0,1]$ has a neighborhood $U$ in which if $u_{t^{\prime}}$ for $t^{\prime} \in U$ is a large solution of the equation $\Delta_{g\left(t^{\prime}\right)} u-\psi(u)=0$, then for any $t^{\prime \prime} \in U$ the equation $\Delta_{g\left(t^{\prime \prime}\right)} u-\psi(u)=0$ has a large solution $u_{t^{\prime \prime}}$ comparable to $u_{t^{\prime}}$. As $[0,1]$ is a compact set it follows that this property holds for $U=[0,1]$, in particular for the endpoint metrics $g$ and $g^{\prime}$.

Corollary 9.2. Let $\psi$ be a function satisfying the Harnack assumptions. Also assume that all domains $\Omega$ with nonempty boundary are $\psi$-regular for a $C^{2}$ metric tensor $g$ on $M$.

Then all domains $\Omega$ with nonempty boundary are $\psi$-regular in any $C^{2}$ metric tensor on $M$.

In addition, if there exists a Riemannian manifold $N$ with $C^{2}$ metric tensor and a point $p$ such that $\Delta u-\psi(u)=0$ has two large solutions $u_{1}, u_{2}$ on the domain $N \backslash\{p\}$ which are not comparable near 0 , then all domains $\Omega \subset M$ such that $M \backslash \Omega$ contains an isolated point are not $\psi$-unique. This holds for any compact Riemannian manifold $M$ without boundary and any $C^{2}$ metric tensor on $M$.

Example 9.1. Let $\psi(u)=u^{\alpha}$ and $\alpha<n /(n-2)$, where $n=\operatorname{dim} M$. Then any domain $\Omega$ on a $C^{2}$ Riemannian manifold $M$ is $u^{\alpha}$-regular and has a unique solution with maximal rate of blowup. If $M \backslash \Omega$ contains an isolated point, then $\Omega$ is not $u^{\alpha}$-unique.

Proof. The first part of the claim that $\Omega$ is $u^{\alpha}$-regular has already been established in Example 3.2. We give another proof which also shows that there is a solution with maximal rate of blowup.

Indeed, the function $u(x)=C|x|^{\beta}$ for $C>0$ solving $C^{\alpha-1}=\beta(\beta+n-2)$ and $\beta=-2 /(\alpha-1)$ is a $u^{\alpha}$-barrier (Definition 2.10) for domain $\mathbb{R}^{n} \backslash\{0\}$. By translation, a similar barrier exists for any point $x \in \mathbb{R}^{n}$.

Now consider $\Omega=M \backslash\{x\}$ for some $x \in \Omega$. Picking local geodesic coordinates centered at $x$ we get a metric tensor $g_{i j}^{\prime}$ satisfying (d) of Proposition 9.1 for $g_{i j}=\delta_{i j}$ in a small neighborhood $U$ of $x$. Hence by Proposition 9.1 since $u$ is a $u^{\alpha}$-barrier on $\mathbb{R}^{n}$ for 0 there exists a local $u^{\alpha}$-barrier $v$ for $x$ on $M$. 
As $x$ was an arbitrary point, it follows from Theorem 3.3 that any domain $\Omega \subset M$ with nonempty boundary is $u^{\alpha}$-regular. Uniqueness of the solution with maximal rate of blowup has been established in Theorem 3.4. If $\Omega$ has an isolated point, then as in Example 3.2 we can construct near this point a whole family of large solutions, so $\Omega$ is not $\psi$-unique.

9.1. Two dimensional uniformization theorem. We reprove a classical result on uniformization of Riemannian surfaces. We would like to refer the reader to a much more detailed treatment in [32, where more general cases are treated.

Corollary 9.3 (Uniformization theorem for domains on a compact manifold $M$ ). Let $M$ be a two dimensional compact connected Riemannian manifold without boundary with $C^{2}$ metric tensor $g$ and let $\Omega$ be a domain on $M$. If the Euler characteristic of $\chi(M)$ is less than or equal to zero and $M \backslash \Omega$ contains at least one point or if $\chi(M)=2$ and $M \backslash \Omega$ contains at least three points, then $\Omega$ can be equipped with a complete metric tensor (called the Poincaré metric) conformal to $g$ of constant Gauss curvature -1. The metric tensor is also unique.

Proof. Notice first that the equation

$$
\Delta u-e^{2 u}=0
$$

has an explicit large positive solution in $B(0,1) \backslash\{0\}$ (Poincaré metric in punctured disk), given by

$$
e^{2 u(r)}=\left(r \log \frac{1}{r}\right)^{-2}, \quad \text { for } r=|x| .
$$

In general, given $g$ on $M$ we consider a new metric tensor $g^{\prime}$ on $M$ to be given by $g^{\prime}=e^{2 u} g$, where $u$ is a large solution of the equation

$$
\Delta u-e^{2 u}=K(x) .
$$

Here $K$ is the Gauss curvature function for the original metric tensor $g$; i.e., $K \in$ $L^{\infty}(\Omega)$. A simple computation shows that if $u$ solves this equation, then $g^{\prime}$ has Gauss curvature -1 .

The equation is not exactly what we have studied so far, as $u$ is allowed to be negative and also $e^{2 u}$ does not satisfy all the necessary assumptions. We deal with the matter now.

First assume that $M \backslash \Omega$ is a set of positive measure. Then it is possible to extend $K$ defined on $\Omega$ onto the whole $M$ such that $K \in L^{\infty}(M)$ and $\int_{M}(K+1) d \mathrm{Vol}=0$. Hence there exists $u_{0}$ defined on $M$ solving $\Delta u_{0}=K+1$. As $u_{0}$ is determined up to a constant we may assume that $u_{0} \leq 0$. Writing $u=u_{0}+v$ we see that $v$ solves

$$
\Delta v-e^{2 u_{0}}\left(e^{2 v}-1\right)=e^{2 u_{0}}-1 \leq 0 .
$$

This equation has the required form as $\psi(v)=e^{2 v}-1$ satisfies all the necessary assumptions, $k(x)=e^{2 u_{0}}$ is positive, continuous and bounded and $f=e^{2 u_{0}}-1<0$. Hence (159) has a positive large solution $v$ in $\Omega$. Next we claim that $u=u_{0}+v$ gives rise to a complete metric on $\Omega$. Indeed by Proposition 9.1 part (d) we can compare the solution of $\Delta w-c \psi(w)=0$ ( $c$ large) at a point $x \in \partial \Omega$ with the solution of (157) which yields by (158) that $\exp (2 w(y)) \geq\left(r \log \frac{1}{r}\right)^{-2}$, where $r=\operatorname{dist}(y, x)$. Comparing $v$ and $w$ gives us that $v \geq w-C$ for some large $C>0$; hence

$$
e^{2 u(y)} \geq\left(\min _{M} e^{2 u_{0}}\right) e^{2 v(y)} \geq K e^{2 w(y)} \geq K\left(r \log \frac{1}{r}\right)^{-2}, \quad \text { for } r=\operatorname{dist}(x, y) .
$$


This is sufficient to show that the new metric $g^{\prime}=e^{2 u} g$ is complete as $\left(\left(r \log \frac{1}{r}\right)^{-2}\right)^{2}$ is not integrable on the interval $[0, \varepsilon]$.

It remains to deal with the case that $M \backslash \Omega$ has measure zero. Let $\chi(M)$ be the Euler characteristic of $M$. If $\chi(M)<0$, then by the Gauss-Bonnet formula we can find $c_{0} \in \mathbb{R}$ such that $\int_{M}\left(K+e^{2 c_{0}}\right) d \mathrm{Vol}=0$. We then proceed as above by finding $u_{0} \leq c_{0}$ such that $\Delta u_{0}=K+e^{2 c_{0}}$. Then $v=u-u_{0}$ solves an equation similar to (160) with nonpositive right hand side and our argument goes through.

If $\chi(M)=2$, then $M$ is conformally equivalent to the Riemann sphere. Indeed, as noted in 32, given a distribution $\delta^{\prime}$ of order 1 at $p \in M$ such that $\left\langle 1, \delta^{\prime}\right\rangle=0$ (a derivative of the delta function) we can solve $\Delta u=\delta^{\prime}$. This can be done on any compact connected $M$, but in this case $M \backslash\{p\}$ is simply connected. Hence $u$ is a real part of a meromorphic function $f$ on $M$ with one simple pole (at $p$ ). Then $f$ defines a holomorphic map $f: M \rightarrow \hat{\mathbb{C}}$, where $\hat{\mathbb{C}}$ is the Riemann sphere. We see that $f$ has degree one, and it follows that $f$ is a holomorphic diffeomorphism. Now, $\hat{\mathbb{C}} \backslash\{\infty\}$ is holomorphically equivalent to $\mathbb{C}$. By removing two additional points (recall that we assume that $M \backslash \Omega$ has at least three points) we get that $M \backslash\left\{p_{1}, p_{2}, p_{3}\right\}$ is holomorphically equivalent to $\mathbb{C} \backslash\left\{q_{1}, q_{2}\right\}$. However, by Lemma 4.3 of [32, such a region has Poincaré metric. Hence so does $M \backslash\left\{p_{1}, p_{2}, p_{3}\right\}$. Let $v$ be the function that give rises to the Poincaré metric on $M \backslash\left\{p_{1}, p_{2}, p_{3}\right\}$ via $g^{\prime}=e^{2 v} g$. We use $v$ as a subsolution. Indeed, let $\Omega_{1} \Subset \Omega_{2} \Subset \cdots \Subset \Omega$ be an increasing sequence of domains approximating $\Omega$. As $M \backslash \Omega_{n}$ has positive measure it follows from the argument above that (159) has a large solution $u_{n}$ on $\Omega_{n}$. By the comparison lemma that can be established for this equation it follows that $u_{1} \geq u_{2} \geq \ldots$ and $u_{n} \geq v$. From this it follows that $u=\lim _{n \rightarrow \infty} u_{n}$ is well defined on $\Omega$ and solves (159) there. Also as $u \geq v$ we get that no geodesic $\gamma: I \rightarrow \Omega$ in the metric $g^{\prime}=e^{2 u} g$ can exit $\Omega$ at the points $p_{1}, p_{2}, p_{3}$, since $v$ is a complete metric on $M \backslash\left\{p_{1}, p_{2}, p_{3}\right\}$. As these three distinct points can be chosen arbitrarily from the set $M \backslash \Omega$, it follows that no geodesic $\gamma: I \rightarrow \Omega$ can exit $\Omega$, i.e., $g^{\prime}$ is complete.

If $\chi(M)=0$, then $M$ is conformal to a flat torus; in this case we can solve the equation $\Delta u=K$ on $M$ giving us a new metric $e^{2 u} g$ which is flat. Hence we may assume that $M=\mathbb{C} / \Lambda$ and $\mathbb{C}$ is a covering space of $M$. We work for now in the covering space. Here we can view $M$ as a parallelogram $\mathcal{P} \subset \mathbb{C}$ whose sides are identified in the standard way and 0 is its interior point. As $\mathbb{C} \backslash \mathcal{P}$ has positive measure, what we have established above and also part (iii) of Theorem 6.2 implies that we can find a solution $\tilde{v}$ of (159) with $K=0$ in $\mathcal{P} \backslash\{0\}$ which blows up at 0 and vanishes on $\partial \mathcal{P}$. It follows that $\tilde{v}$ attains its minimum at the boundary of $\mathcal{P}$; hence $\frac{\partial \tilde{v}}{\partial \nu}(y) \leq 0$ for all $y \in \partial \mathcal{P}$. Here $\nu$ is the outer normal to $\mathcal{P}$ at the point $y$. It follows that if we push down $\tilde{v}$ back to $M$ we get $v$ on $M \backslash\{p\}$ a large subsolution of (159) with $K=0$. Again by repeating the construction we gave above, i.e., we approximate $\Omega$ by a sequence of increasing domains $\Omega_{n}$, we obtain a nonincreasing sequence of solutions $u_{n}$ for which $v$ serves as a subsolution if $p \in M \backslash \Omega$. Hence $u=\lim _{n \rightarrow \infty} u_{n}$ is well defined, solves our equation and gives rise to a complete metric.

As far as the uniqueness goes we refer to the discussion in 32 .

9.2. Maximal rate of blowup for $\psi(u)=u^{\alpha}$. We present several sufficient and necessary conditions that guarantee the existence of a solution of the equation (162) with maximal rate of blowup (163). These conditions are more general than present 
in the current literature and in particular generalize conditions in [14] and [15]. We note at this place that the paper 24] does not deal with the issue of the rate of blowup of the maximal solution.

Proposition 9.4. Let $M$ be a compact Riemannian manifold with $C^{2}$ metric tensor $g$ and let $\Omega \subset M$ be a domain with nonempty boundary. Consider $\psi(u)=u^{\alpha}$ for some $\alpha>1$. Let $\tilde{h}, k, f \in L^{\infty}(\Omega), f \geq 0$ and $k \geq c>0$. Then the equation

$$
\Delta_{g} u+\tilde{h} u-k u^{\alpha}=-f \quad \text { in } \Omega
$$

has at most one large positive solution $u$ with maximal rate of blowup, i.e., a solution that satisfies

$$
\liminf _{\delta(x) \rightarrow 0+} \delta(x)^{2 /(\alpha-1)} u(x)>0 .
$$

Such a solution exists if and only if every boundary point $x \in \partial \Omega$ has an open neighborhood $U$ and $C^{2}$ metric tensor $h$ in $U$ that is conformal to $g$ at $x$, such that the equation $\Delta_{h} v-v^{\alpha}=0$ has a positive solution in $U \cap \Omega$ satisfying

$$
\liminf _{y \rightarrow x} \delta_{h}(y)^{2 /(\alpha-1)} v(y)>0 .
$$

Proof. In (164) we wrote $\delta_{h}$ to emphasize that we consider there the distance function generated by the metric $h$. However, as both $g$ and $h$ are $C^{2}$ metric tensors near $x$, the distance functions they generate are equivalent near $x$; hence $\delta_{g} \approx \delta_{h}$ and we can drop the subscript. The key here is Proposition 9.1. It is enough to show that the equation $\Delta_{g} u-u^{\alpha}=0$ has a large solution with maximal rate of blowup. If this is true, then the same is true for the equation $\Delta_{g} u-c u^{\alpha}=0$ for any $c>0$; i.e., $\Omega$ is a $u^{\alpha}$-regular domain. Hence (162) has the largest solution (call it $\left.U_{\max }\right)$ for which a solution of $\Delta_{g} u-c u^{\alpha}=0$ serves as a subsolution for large $c>0$ near $\partial \Omega$.

From this we obtain that $U_{\max }$ also has a maximal rate of blowup. Uniqueness follows from Proposition 3.4 whose proof goes without change even for the more general equation (162) (notice that we do not need continuity of $k$ at the boundary $\partial \Omega)$.

Assume therefore that $u$ is the largest solution of the equation $\Delta_{g} u-\psi(u)=0$ in $\Omega$ and assume that (163) does not hold, i.e., that there exists a point $x \in \partial \Omega$ such that

$$
\liminf _{y \rightarrow x} \delta(y)^{2 /(\alpha-1)} u(y)=0 .
$$

As before, consider local geodesic coordinates defined for a small ball $U$ centered at $x$; in these coordinates the metric tensor $g$ takes the form $g_{i j}(x)=\delta_{i j}(x)$ and $\nabla g_{i j}(x)=0$. Now consider the metric tensor $h$ for which we have (164) for the equation $\Delta_{h} v-v^{\alpha}=0$ at $x$. We can express $h$ in the same local coordinates as $g$, giving us a tensor $h_{i j}=k \delta_{i j}$. Consider first the case $k=1$. Then $\|g-h\|_{L^{\infty}(U)} \leq$ $C \operatorname{diam}(U)$; i.e., this quantity can be made arbitrarily small by taking $U$ small. On the other hand, $\|\nabla(g-h)\|_{L^{\infty}(U)} \leq C$; i.e., $g$ and $h$ are not necessarily close in the Lipschitz norm. Looking back at the proof of Proposition 9.1 we realize that we do not need this as, since (a) holds for $v$, then $|\nabla v| \leq C \delta \psi(v)$. Hence if $\operatorname{diam}(U)<\varepsilon$ this, after being plugged into (152), yields (153) for $g$ and $h$. This is enough to see that $v$ is a subsolution of the equation $\Delta_{g} u-c u^{\alpha}=0$ in $U \cap \Omega$ for small $c>0$. Having this, the equation must have at least one solution $w$ larger that $v$, i.e., with maximal rate of blowup. Scaling properties of our equation then imply that 
$w / \theta$ for some $\theta>1$ large solves $\Delta_{g} u-u^{\alpha}=0$ in $U \cap \Omega$. Let $W$ be the largest solution of the same equation in $U$. Then $\frac{1}{2}(w / \theta+W)$ is a large subsolution of the equation $\Delta_{g} u-u^{\alpha}=0$ in $U \cap \Omega$. Hence $\frac{1}{2}(w / \theta+W) \leq u+W$, as $u+W$ is a large supersolution of the same equation. It follows that

$$
\liminf _{y \rightarrow x} \delta(y)^{2 /(\alpha-1)} u(y) \geq \frac{1}{2 \theta} \liminf _{y \rightarrow x} \delta(y)^{2 /(\alpha-1)} w(y)>0,
$$

which contradicts (165). From this our claim follows. If $k \neq 1$ all we wrote above is true for the metric tensor $h_{i j}^{\prime}=h_{i j} / k$. This is enough, as any solution of $\Delta_{h} u-u^{\alpha}=$ 0 is a solution of $\Delta_{h^{\prime}} u-c u^{\alpha}=0$ for some $c=c(k)>0$, i.e., all that holds for $h$ holds for $h^{\prime}$ as well.

Definition 9.5. Let $\Gamma$ be a set in $\mathbb{R}^{n}$ and let $p \in \Gamma$. For given $\varepsilon>0$ let $S_{* p}^{\varepsilon} \Gamma$ be the set

$$
S_{* p}^{\varepsilon} \Gamma=\left\{\omega \in \mathbb{S}^{n-1}: p+t \omega \in \Gamma \text { for all } t \in[0, \varepsilon)\right\} .
$$

Definition 9.6. Assume that $\alpha>1$ and $\varepsilon>0$. Let $\Sigma \subset M$ be a nonempty set inside a compact, connected, boundaryless Riemannian manifold $M$ of dimension $n \geq 2$ with $C^{2}$ metric tensor $g$. Let $k=0,1, \ldots, n-2$ be the smallest number for which $\alpha<(n-k) /(n-k-2)$. We say that the set $\Sigma$ is $(\alpha, \varepsilon)$-regular if either $k=0$ or the following holds:

There exists a dense set $\Sigma^{\prime}$ in $\Sigma$ such that every point $p \in \Sigma^{\prime}$ has a $C^{2}$ diffeomorphism $\phi: U=B(p, \varepsilon) \rightarrow V$ from a geodesic ball $B(p, \varepsilon)$ to a neighborhood $V$ of 0 in $\mathbb{R}^{n}$ such that

(a) $\phi(p)=0$.

(b) $|\nabla \phi| \leq 1 / \varepsilon,\left|\nabla \phi^{-1}\right| \leq 1 / \varepsilon$.

(c) $g_{i j}(p)=\phi^{*}\left(\delta_{i j}(0)\right)$ (here $\phi^{*}$ denotes the pull-back of the flat metric on $\mathbb{R}^{n}$ ).

(d) The set $S_{0 *}^{\varepsilon^{2}}(\phi(V \cap \Gamma))$ has $(\alpha, \varepsilon)$-admissible subset on $\mathbb{S}^{n-1}$.

Remark 9.1. The fact that $\phi$ needs to be $C^{2}$ is an artificial leftover of our assumption that the metric tensor on $M$ is $C^{2}$. In fact the definition above requires only uniform control of the Lip norm of $\phi$ and $\phi^{-1}$; i.e., $\phi$ needs to be bi-Lipschitz. There is no control required on the norm of $\nabla^{2} \phi$. It is possible (with few complications) to develop the whole theory on the existence and uniqueness for the Lipschitz metric tensor; in that case this definition can be stated without assuming $\phi$ is $C^{2}$.

Proposition 9.7. Let $M$ be a compact Riemannian manifold with $C^{2}$ metric tensor $g$ and let $\Omega \subset M$ be a domain with nonempty boundary. Let $\alpha>1$. Finally, let $h, k, f \in L^{\infty}(\Omega), f \geq 0$ and $k \geq c>0$. If the set $M \backslash \Omega$ is $(\alpha, \varepsilon)$-admissible for some $\varepsilon>0$, then the equation

$$
\Delta_{g} u+h u-k u^{\alpha}=-f \quad \text { in } \Omega
$$

exactly one large positive solution $u$ with maximal rate of blowup. There exists $c(\varepsilon)>0$ such that

$$
u(x) \delta(x)^{2 /(\alpha-1)} \geq c(\varepsilon) \quad \text { for all } x \in \Omega .
$$

Proof. Let $\Gamma=M \backslash \Omega$ and assume that $\Gamma$ is $(\alpha, \varepsilon)$-admissible. It suffices to show that the largest solution $U_{\max }$ of the equation $\Delta u-u^{\alpha}=0$ satisfies (169). We proceed by an induction in $k$. The case $k=0$ has been dealt with in Example 8.1. It also follows that in this case the constant $c(\varepsilon)>0$ does not depend on $\varepsilon>0$, but only on the manifold $M$ and its metric. 
Assume therefore that we already have our theorem for $k-1$. Consider any point $p$ from the dense subset of $\Gamma$ for which (a)-(d) of Definition 9.6 holds. Hence there is a set $\emptyset \neq \Sigma \subset S_{0 *}^{\varepsilon^{2}}(\varphi(V \cap \Gamma))$ that is $(\alpha, \varepsilon)$-admissible on $\mathbb{S}^{n-1}$; i.e., by the inductive assumption we know that there exists a function $\omega$ on $\mathbb{S}^{n-1} \backslash \Sigma$ solving

$$
\Delta_{\mathbb{S}^{n-1}} \omega-\frac{2}{\alpha-1}\left(n-2-\frac{2}{\alpha-1}\right) \omega-\omega^{\alpha}=0,
$$

and $\omega(x) \operatorname{dist}_{\mathbb{S}^{n-1}}(x, \Sigma)^{2 /(\alpha-1)} \geq c_{0}(\varepsilon)>0$ for all $x \in \mathbb{S}^{n-1} \backslash \Sigma$. It follows (via polar coordinates) that $v(x)=v(r \omega)=r^{-2 /(\alpha-1)} \omega$ is a large solution of $\Delta v-v^{\alpha}=0$ in the cone $\mathcal{C}=\left\{x=r \omega \in \mathbb{R}^{n}: r \geq 0\right.$ and $\left.\omega \in \mathbb{S}^{n-1} \backslash \Sigma\right\}$. Here the background metric is flat $\mathbb{R}^{n}$. Also $v(x) \operatorname{dist}_{R^{n}}(x, \partial \mathcal{C})^{2 /(\alpha-1)} \geq c_{1}(\varepsilon)>0$ for all $x \in V \cap \mathcal{C}$.

Let us pull back the metric tensor from $V$ to $U$ and denote it by $h$. Consider $V^{\prime}=B(p, \delta)$ for $0<\delta \leq \varepsilon$, which will be specified later. The assumptions on $h$ imply that $\|g-h\|_{L^{\infty}\left(V^{\prime}\right)} \leq \frac{\delta}{\varepsilon}$ and $\|\nabla h\|_{L^{\infty}\left(V^{\prime}\right)} \leq \frac{1}{\varepsilon}$. This, as follows from Proposition 9.4. suffices for applying part (a) of Proposition 9.1, provided $\delta>0$ is chosen sufficiently small. As the proof shows the choice of $\delta>0$ depends on $\varepsilon$, the metric tensor $g$ and the constant $c_{1}(\varepsilon)>0$. (We need $c_{1}(\varepsilon)$ for the estimate $v / \delta^{2} \leq c_{1}^{\alpha-1} v^{\alpha}$.) So, $\delta=\delta(\varepsilon)>0$.

Hence part (a) of Proposition 9.1 applies implying that $w=\phi^{-1}(v)$ is a solution of the equation $\Delta_{h} w-w^{\alpha}=0$ and a subsolution of $\Delta_{g} u-\frac{1}{2} u^{\alpha}=0$. Also,

$$
w(x) \operatorname{dist}_{g}\left(x, \phi^{-1}(\partial \mathcal{C})\right)^{2 /(\alpha-1)} \geq c_{2}(\varepsilon)>0 \quad \text { for all } x \in U \backslash \phi^{-1}(\mathcal{C}) \subset \Omega .
$$

Using scaling, the equation has a multiple of $w$, say $W$, that is a subsolution of $\Delta_{g} u-u^{\alpha}=0$ and $W$ satisfies (171) with a different constant $c_{3}(\varepsilon)>0$. We use $W$ to compare it with $U_{\max }$. For every point $x \in M$ we denote by $u_{x}$ the large solution of the equation $\Delta_{g} u-u^{\alpha}=0$ in the geodesic ball $B(x, \delta)$. As the metric tensor $g$ is uniformly elliptic and $C^{2}$ on $M$ we conclude that there is a constant $K=K(\delta, g)>0$ such that $u_{x} \leq K$ on $B(x, \delta / 2)$.

Hence, as $W$ is a subsolution of our equation in $B(p, \delta) \cap \Omega$ and $U_{\max }+u_{p}$ is a large supersolution of the same equation in $B(p, \delta) \cap \Omega$, we see that $W \leq U_{\max }+u_{p}$ on $B(p, \delta) \cap \Omega$. From this, $U_{\max } \geq W-K$ on $B(p, \delta / 2) \cap \Omega$. As $W$ satisfies (171) with constant $c_{3}(\varepsilon)>0$ we finally conclude that for some $0<\delta_{0}=\delta_{0}\left(c_{3}(\varepsilon), K(\varepsilon)\right) \leq \delta / 2$ and all $x \in B\left(p, \delta_{0}\right) \cap \Omega$,

$$
\begin{aligned}
U_{\max }(x) \operatorname{dist}(x, p)^{2 /(\alpha-1)} & \geq w(x) \operatorname{dist}_{g}\left(x, \phi^{-1}(\partial \mathcal{C})\right)^{2 /(\alpha-1)}-K \operatorname{dist}(x, p)^{2 /(\alpha-1)} \\
(172) & \geq c_{3}(\varepsilon) / 2>0 .
\end{aligned}
$$

Recall that this holds for a dense set of $p \in \Gamma$. This is enough to see that

$$
U_{\max }(x) \delta^{2 /(\alpha-1)}(x) \geq c_{3}(\varepsilon) / 2>0, \quad \text { for all } x \in \Omega \text { with } \delta(x)<\delta_{0}(\varepsilon) .
$$

Finally by the Harnack inequality (Lemma 4.5) there exists a $C=C\left(\delta_{0}, M\right)>0$ such that for all $x$ with $\delta(x) \geq \delta_{0}$ :

$$
U_{\max }(x) \geq C\left(\delta^{\prime}\right) U_{\max }(y), \quad \text { for some } y \text { such that } \operatorname{dist}(x, y)=\delta_{0} .
$$

Putting (172) and (174) together yields that there exists $c>0$ depending only on $\varepsilon>0$, the manifold $M$ and the metric tensor $g$ such that

$$
U_{\max }(x) \delta^{2 /(\alpha-1)}(x) \geq c>0, \quad \text { for all } x \in \Omega .
$$

This finishes our proof, as the uniqueness was established before. 
Corollary 9.8. Let $M$ be a compact Riemannian manifold with $C^{2}$ metric tensor $g$ and let $\Gamma$ be a smooth submanifold (with or without boundary) of dimension d. Then equation (168) has a large positive solution $u$ in $\Omega=M \backslash \Gamma$ with maximal rate of blowup, provided $\alpha<(n-d) /(n-d-2)$.

The corollary is a classical result which follows immediately from our Proposition 9.7 .

9.3. Uniqueness for $\psi(u)=u^{\alpha}$. We dedicate our last proposition to $u^{\alpha}$-uniqueness. The following result is somewhat unsatisfactory as it leaves open a small gap between examples we have for nonuniqueness (essentially $d=\operatorname{dim}[(M \backslash \Omega)$ $\cap B(x, r)] \leq n-2$ for one point $x \in \partial \Omega$ and some $r>0)$ and uniqueness $d \geq n-1$. Moreover, this lemma needs some (very likely unnecessary) smoothness assumptions on the $(n-1)$-dimensional surface (there are no such assumptions in Proposition 3.8 which on the other hand work only for special manifolds $M$ ). In fact we conjecture:

Conjecture. If at every point $x \in \Gamma$ there exists $d>n-2$ such that

$$
\liminf _{r \rightarrow 0+} r^{-d} H^{d}(\Gamma \cap B(x, r))>0,
$$

then $\Omega=M \backslash \Gamma$ is $u^{\alpha}$-unique.

Proposition 9.9. Assume that $\alpha>1$ and $\varepsilon>0$. Let $M$ be a compact Riemannian manifold with $C^{2}$ metric tensor $g$ and let $\Omega \subset M$ be a domain with nonempty boundary. Denote by $\Sigma=M \backslash \Omega$. Let $\Sigma$ be such that every point $p \in \Sigma$ has a biLipschitz bijection $\phi: U=B(p, \varepsilon) \rightarrow V$ from a geodesic ball $B(p, \varepsilon)$ to a neighborhood $V$ of 0 in $\mathbb{R}^{n}$ such that

(a) $\phi(p)=0$.

(b) $|\nabla \phi| \leq 1 / \varepsilon,\left|\nabla \phi^{-1}\right| \leq 1 / \varepsilon$.

(c) $g_{i j}(p)=\phi^{*}\left(\delta_{i j}(0)\right)$ (here $\phi^{*}$ denotes the pull-back of the flat metric on $\mathbb{R}^{n}$ ).

(d) The set $S_{0 *}^{\varepsilon^{2}}(\phi(V \cap \Gamma))$ contains the set

$$
S_{q, v}=\left\{x=\left(x_{1}, x_{2}, \ldots, x_{n}\right) \in \mathbb{S}^{n-1}: \operatorname{dist}(x, q)<\varepsilon \text { and } x \perp v\right\}
$$

for some $q \in \mathbb{S}^{n-1}$ and $v$ a unit vector such that $v \perp q . \quad\left(S_{q, v}\right.$ is an intersection of $(n-1)$-dimensional hyperplane in $\mathbb{R}^{n}$ with a spherical cap on $\mathbb{S}^{n-1}$.)

Then $\Omega$ is a $u^{\alpha}$-unique domain. Moreover, the large solution of equation (168) has a maximal rate of blowup.

Remark 9.2. Proposition 9.9 translated into normal language means that $\Gamma$ contains near each of its points a sufficiently large piece of an $(n-1)$-dimensional Lipschitz surface with corners of aperture bounded from below. Proposition 9.9 can also be formulated locally; i.e., if a point $x \in \partial \Omega$ has a neighborhood $U$ in which $U \cap \Omega$ satisfies the assumptions of Proposition 9.9, then $x$ is $\psi$-unique.

Proof. As $\Sigma$ satisfies the assumptions of Proposition 9.7 (note Remark 9.1, although in this simpler case a rigorous argument that $\phi$ suffices to be bi-Lipschitz can be made without too much trouble) there is a unique large solution with maximal rate of blowup. Let us denote by $U_{\max }$ such a solution. We want to show it is the only solution. For $p \in \partial \Omega$ consider the set

$$
\mathcal{T}_{p}=\phi^{-1}\left(T_{p}\right) \subset \Gamma, \quad \text { where } \quad T_{p}=\left\{(r, \omega) \in V: 0<r<\varepsilon^{2} \text { and } \omega \in S_{q, v}\right\} .
$$


Notice that $p \in \overline{\mathcal{T}_{p}} \backslash \mathcal{T}_{p}$. If $y \in \mathcal{T}_{p}$ there are two possibilities. Either $y$ is an interior point of $\Gamma$ and in such a case all is fine, or $y \in \partial \Omega$ and in that case we claim that any large solution $u$ in $\Omega$ has maximal blowup at $y$. Indeed, in this case, $\mathcal{T}_{p}$ splits $\Omega$ into two parts on a neighborhood of $y$, since $\mathcal{T}_{p}$ is a hypersurface. Hence for any point $z \in \Omega$ on one side of this hypersurface we can compare a large solution $u$ on the same side as $z$ with large solutions with maximal blowup outside cones of uniform aperture nearly touching $\mathcal{T}_{p}$ from the other side. Without going into too many details this gives $u(z) \operatorname{dist}\left(z, \mathcal{T}_{p}\right)^{2 /(\alpha-1)} \geq c_{0}>0$ near $y$ with $c_{0}$ not depending on $y$.

We move back to point $p$. For $t \in[0, \delta)$ consider

$$
\mathcal{T}_{p}^{t}=\phi^{-1}\left(T_{p}+t q\right)
$$

We see that $\mathcal{T}_{p}^{0}=\mathcal{T}_{p}$ and $\overline{\mathcal{T}_{p}^{t}} \subset \mathcal{T}_{p}$ for $t>0$. So $p$ belongs to $M \backslash \overline{\mathcal{T}_{p}^{t}}$. We find a large solution $w^{t}$ with maximal rate of blowup on the domain $M \backslash \frac{p}{\mathcal{T}_{p}^{t}}$ and compare it with $u$, i.e., any large solution on $\Omega$. The only "bad" points where anything can go wrong are points $y$ such that $y \in \partial \Omega \cap \partial\left(M \backslash \overline{\mathcal{T}_{p}^{t}}\right)$. However at such a point we know we have $c_{1} \geq w^{t}(z) \operatorname{dist}\left(z, \mathcal{T}_{p}^{t}\right)^{2 /(\alpha-1)} \geq c_{2}$ by Proposition 9.7 for some $c_{1}, c_{2}>0$ depending only on $\varepsilon>0$. This gives that near point $y: u(z) \geq c_{0} / c_{1} w^{t}(z)$. We can assume that $c_{0} / c_{1}<1$. At all other points $y \in \partial \Omega$ we have that $u(y)$ is infinite but $w^{t}(y)$ is finite. If follows that $\left.u\right|_{\partial \Omega} \geq c_{0} /\left.c_{1} w^{t}\right|_{\partial \Omega}$. Hence by the comparison lemma, $u \geq c_{0} / c_{1} w^{t}$ everywhere. Finally, letting $t \rightarrow 0+$ implies that $u \geq c_{0} / c_{1} w^{0}$. As $w^{0}$ has maximal rate of blowup at $p$ we get that $u(z) \operatorname{dist}(z, p)^{2 /(\alpha-1)} \geq c_{3}>0$ for a constant $c_{3}$ independent of the particular point $p$. This looks like (172) and indeed implies that $u$ has maximal rate of blowup. As there is only one solution with maximal rate of blowup we get that $u=U_{\max }$. So uniqueness follows.

\section{REFERENCES}

[1] P. Aviles, A study of the singularities of solutions of a class of nonlinear elliptic partial differential equations, Comm. PDE 7 (1982), no. 6, 609-643. MR660747 (84j:35070)

[2] P. Aviles and R. McOwen, Conformal deformations of complete manifolds with negative curvature, J. Differential Geom. 21 (1985), no. 2, 269-281. MR816672 (87e:53058)

[3] P. Aviles and R.McOwen, Conformal deformation to constant negative scalar curvature on noncompact Riemannian manifolds, J. Differential Geom. 27 (1988), no. 2, 225-239. MR925121(89b:58225)

[4] P. Aviles and R. McOwen, Complete conformal metrics with negative scalar curvature in compact Riemannian manifolds, Duke Math. J. 56 (1988), no. 2, 395-398. MR932852(89b:58224)

[5] R. Benguria, H. Brezis and E. Lieb, The Thomas-Fermi-Von Weizsaäcker theory of atoms and molecules, Comm. Math. Phys. 79 (1981), 167-180. MR612246 (83m:81114)

[6] P. Delanoë, Generalized stereographic projections with prescribed scalar curvature. Geometry and nonlinear partial differential equations, Contemp. Math. 127, Amer. Math. Soc., Providence, RI (1992), 17-25. MR1155406 (93e:53045)

[7] M. Dindoš, Existence and uniqueness for a semilinear elliptic problem on Lipschitz domains in Riemannian manifolds, Comm. PDE, 27, (2002), 219-291. MR1886960 (2003a:35070)

[8] M.Dindoš, Existence and uniqueness for a semilinear elliptic problem on Lipschitz domains in Riemannian manifolds II, Trans. Amer. Math. Soc. 355 (2003), no. 4, 1365-1399. MR1946396 (2004b:58033)

[9] M. Dindoš, Hardy spaces and potential theory for $C^{1}$ domains in Riemannian manifolds (based on my Ph.D. thesis), Memoirs of AMS 191 (2008), no. 894. MR.2376731|(2009c:42048)

[10] M. Dindoš and M. Mitrea, Semilinear Poisson problems in Sobolev-Besov spaces on Lipschitz domains, Publ. Mat. 46 (2002), no. 2, 353-403. MR1934200 (2003g:35061) 
[11] E. Dynkin and S. Kuznetsov, Fine topology and fine trace on the boundary associated with a class of semilinear differential equations, Comm. Pure Appl. Math. 51 (1998), no. 8, 897-936. MR:1620224 (99f:35046)

[12] E. Dynkin and S. Kuznetsov, Trace on the boundary for solutions of nonlinear differential equations, Trans. Amer. Math. Soc. 350 (1998), no. 11, 4499-4519. MR1422602 (99a:60084)

[13] S. Fakhi, Positive solutions of $\Delta u+u^{p}=0$ whose singular set is a manifold with boundary, Calc. Var. Partial Differential Equations 17 (2003), no. 2, 179-197. MR.1986318(2005c:35094)

[14] D. L. Finn, Existence of positive solutions to $\Delta_{g} u=u^{q}+S u$ with prescribed singularities and their geometric implications, Comm. PDE 23 (1998), no. 9-10, 1795-1814. MR1641725 (2000d:35054)

[15] D. L. Finn, On the negative case of the singular Yamabe problem, J. Geom. Anal. 9 (1999), no. 1, 73-92. MR.1760721 (2001g:53071)

[16] D. L. Finn, Positive solutions of $\Delta_{g} u=u^{q}+$ Su singular at submanifolds with smooth boundary, Indiana Univ. Math. J. 43 (1994), 1359-1397. MR.1322624 (96a:35048)

[17] D. L. Finn, Behavior of positive solutions to $\Delta_{g} u=u^{q}+$ Su with prescribed singularities, Indiana Univ. Math. J. 49 (2000), no. 1, 177-219. MR.1777033 (2001h:53049)

[18] D. L. Finn and R. McOwen, Singularities and asymptotics for the equation $\Delta_{g} u-u^{q}=S u$, Indiana Univ. Math. J. 42 (1993), no. 4, 1487-1523. MR.1266103 (95g:58216)

[19] J.-F. Le Gall, Les solutions positives de $\Delta u=u^{2}$ dans le disque unité. (French), C. R. Acad. Sci. Paris Sér. I Math. 317 (1993), no. 9, 873-878. MR.1246656 (94h:35059)

[20] D. Gilbarg and N. Trudinger, Elliptic Partial Differential Equations of Second Order, 3rd edition, Springer-Verlag, Berlin-New York, 1998.

[21] J. B. Keller, On solutions of $\Delta u=f(u)$, Comm. Pure Appl. Math. 10 (1957), 503-510. MR0091407 (19:964c)

[22] C. Kenig, Harmonic Analysis: Techniques for Second Order Elliptic Boundary Value Problems, American Math. Society, 1994. MR1282720(96a:35040)

[23] N. Korevaar, R. Mazzeo, F. Pacard, R. Schoen, Refined asymptotics for constant scalar curvature metrics with isolated singularities, Invent. Math. 135 (1999), no. 2, 233-272. MR.1666838 (2001a:35055)

[24] D. Labutin, Wiener regularity for large solutions of nonlinear equations, Ark. Math. 41 (2003), 307-338. MR2011924 (2004m:35086)

[25] D. Labutin, Thinness for Scalar-Negative Yamabe Metrics, ArXiv:math/0506226v1 (2005).

[26] C. Loewner and L. Nirenberg, Partial differential equations invariant under conformal or projective transformations, Contributions to analysis (a collection of papers dedicated to Lipman Bers), pp. 245-272. Academic Press, New York, 1974. MR0358078 (50:10543)

[27] X. Ma and R. McOwen, Complete conformal metrics with zero scalar curvature, Proc. Amer. Math. Soc. 115 (1992), no. 1, 69-77. MR1101988 (92h:53049)

[28] R. Mazzeo, Regularity for the singular Yamabe problem, Indiana Univ. Math. J. 40 (1991), no. 4, 1277-1299. MR.1142715 (92k:53071)

[29] R. Mazzeo and F. Pacard, Constant scalar curvature metrics with isolated singularities, Duke Math. J. 99 (1999), no. 3, 353-418. MR1712628 (2000g:53035)

[30] R. Mazzeo, D. Pollack and K. Uhlenbeck, Moduli spaces of singular Yamabe metrics, J. Amer. Math. Soc. 9 (1996), no. 2, 303-344. MR.1356375 (96f:53055)

[31] R. Mazzeo, D. Pollack and K. Uhlenbeck, Connected sum constructions for constant scalar curvature metrics., Topol. Methods Nonlinear Anal. 6 (1995), no. 2, 207-233. MR.1399537 (97e:53076)

[32] R. Mazzeo and M. Taylor, Curvature and uniformization, Israel J. Math. 130 (2002), 323-346. MR.1919383 (2003j:30063)

[33] R. McOwen, Singularities and the conformal scalar curvature equation. Geometric analysis and nonlinear partial differential equations, 221-233, Lecture Notes in Pure and Appl. Math. 144, Dekker, New York (1993). MR.1207185 (94b:53076)

[34] R. McOwen, Negative curvature and conformal deformations of complete manifolds. Nonlinear problems in geometry (Mobile, Ala., 1985), 93-98, Contemp. Math., 51, Amer. Math. Soc., Providence, RI, 1986. MR848936 (87h:58228)

[35] M. Mitrea and M. Taylor, Boundary layer methods for Lipschitz domains in Riemannian manifolds, J. Funct. Anal. 163 (1999), 181-251. MR1680487 (2000b:35050) 
[36] M. Mitrea and M. Taylor, Potential theory on Lipschitz domains in Riemannian manifolds: $L^{p}$, Hardy and Hölder space results, Communications in Analysis and Geometry 9 (2001), no. 2, 369-421. MR.1846208 (2002f:31012)

[37] M. Mitrea and M. Taylor, Potential theory on Lipschitz domains in Riemannian manifolds: Sobolev-Besov space results and the Poisson problem, J. Funct. Anal., 176 (2000), 1-79. MR:1781631 (2002e:58044)

[38] M. Mitrea and M. Taylor, Potential theory on Lipschitz domains in Riemannian manifolds: the case of Hölder continuous metric tensors, Communications in PDE, Vol.25, No.7-8 (2000), 1487-1536. MR1765156 (2001h:35040)

[39] M. Marcus and L. Véron, Uniqueness and asymptotic behaviour of solutions with boundary blow-up for a class of nonlinear elliptic equations, Ann. Inst. Henri Poincaré, Vol. 14, No. 2 (1997), 237-274. MR1441394 (97m:35068)

[40] M. Marcus and L. Véron, The boundary trace of positive solutions of semilinear elliptic equations: the subcritical case, Arch. Rational Mech. Anal. 144 (1998), no. 3, 201-231. MR.1658392 (2000a:35077)

[41] M. Marcus and L. Véron, The boundary trace of positive solutions of semilinear elliptic equations: the supercritical case, J. Math. Pures Appl. (9) 77 (1998), no. 5, 481-524. MR1626800 (99g:35045)

[42] M. Marcus and L. Véron, Existence and uniqueness results for large solutions of general nonlinear elliptic equations, J. Evol. Eq. 3 (2004), 637-652. MR.2058055 (2005c:35103)

[43] M. Marcus and L. Véron, Maximal solutions of the equation $\Delta u=u^{q}$ in arbitrary domains, C. R. Acad. Sci. Paris Ser. I344 (2007), 299-304. MR2308115 (2008b:35089)

[44] R. Osserman, On the inequality $\Delta u \geq f(u)$, Pacific J. Math. 7 (1957), 1641-1647. MR0098239 (20:4701)

[45] R. Schoen and S.T.Yau, On the structure of manifolds with positive scalar curvature, Manuscripta Math. 28 (1979), no. 1-3, 159-183. MR535700 (80k:53064)

[46] R. Schoen and S. T. Yau, Conformally flat manifolds, Kleinian groups and scalar curvature, Invent. Math. 92 (1988), no. 1, 47-71. MR931204 (89c:58139)

[47] E. M. Stein, Singular Integrals and Differentiability Properties of Functions, Princeton University Press, 1970. MR0290095 (44:7280)

[48] M. Taylor, Partial Differential Equations, Springer-Verlag, 1996. MR1395147 (98b:35002a)

[49] J. J. Tang, Prescribing curvature on manifolds with nonpositive Yamabe invariant and singularities, Comm. Partial Differential Equations 19 (1994), no. 5-6, 701-718. MR.1274537 (95j:53062)

School of Mathematics and Maxwell Institute for Mathematical Sciences, University of Edinburgh, Mayfield Road, JCMB KB, Edinburgh EH9 3JZ, United Kingdom 